\title{
Bio-inertia Resonates Life into Evolution
}

\author{
Yi Yu Lai \\ Medical Center, Room B343, Medical Science Building, Tsinghua University, Beijing, 100084. PR China
}

\begin{abstract}
Life is an environmental signal compromised "Le Châtelier's system". The potential for it to issue "Le Châtelier's effect" to discernable signals is defined as bio-inertia. To track the origin of such system recovering capability, we composite brachistochrone curves with experiments to prove that a path which can be projecting into three-dimensional simple harmonic motion with internal commutation is an efficient sustaining signal accumulating and transferring system. It is defined as a bio quantum path which composes of the fundamental physical splicing structures of bio-systems. Experiments calibrate "gravitational loss" as empirical bio-inertia. Surface tension inversion is therefore evidently to be the fundamental gravitational binding and polarization pattern in bio-systems. CSF, as the highest gravitational binding system of vertebrates, has also been originated back to the inversion of an earlier phospholipid bilayer based on the ancient somatic physical training. By adapting Chu's constant into Planck's law and the born of the Schrödinger Cat's babies, the fragile "Copenhagen superposition" becomes "converging superposition" that can be modulated by a music frequency notation in enough binding Le Châtelier's system. Inside surface tension regions, quantum mechanics recovers its gravitational quiddity by redefining Schrödinger wave function, and binding Einstein's gravitational waves that regenerate bio-functions get an equivalent definition. The Principle of Least Action is generalized as the relay or harmonic inversion of the multi-surface tension transferring in bio-systems, and the mechanism of gravitational binding agrees with modified Maxwell's equations for volume to area oscillation. From the binding and polarization of gravity on bio quantum paths, life is physically abiogenesis from a whirlpool by surface tension inversions. NKT cell clinical trial based on new definition confirms that ancient spinal training systems are still unsurpassable for precluding of "spinal cancerous recurrent factors" before modern biotechnologies can establish spinal CSF surface tension interference method in the future.
\end{abstract}

Keywords: Bio-inertia, bio quantum path, gravitational binding, multi-surface tension system, inversion, spinal cancerous recurrent factor

\section{Introduction}

Our planet Earth formed into around 4.54 billion years ago [1] and life originated on it approximately 3.5-3.8 billion years ago [2]. Physical evidence has been found in biogenic graphite in 3.7 billion-year-old metasedimentary rocks from south -western Greenland [3] and microbial mat fossils found in 3.48 billion-year-old sandstone from Western Australia [4]. Evolutionary processes and adaptations result in immense biodiversity in the geological era. Existing patterns of the biosphere have been fluctuating by both speciation and extinction. More than $99 \%$ of all species that ever lived on Earth had been extinct [5]. Estimates of Earth's current species range from 10-14 million, of which only about 1.2 million have been documented and over 86 percent have not yet been described [6].

For the driving forces of evolution, the natural selection theory of Charles Darwin is the only "officially" acknowledged cause of adaptation that first published in his book On the Origin of Species (1859) [7]. However, this theory is still a "selfevident" theory [8]; the precise mechanisms of reproductive heritability and the origin of new traits remained a mystery due to it only described what gave the "survivors" and unknown the "motivations" drive species to "survivors". The reason that it became the only acknowledged theory is just modern genetics can easily explain it in a way that fully incompatible with "acquired traits", albeit Darwin himself actually endorsed Lamarckism. Such "hidden modification" still not solve the "unknown" issues. E.g., neural theory [9] has noticed that the strength of inner molecular changes was much more powerful than those of the selection theory required. Also, modern epigenetics in recent decades increasingly reported RNA "acquired traits" [10], despite it is still unclear whether these acquired traits could have degenerated into inheritable sequences. Most importantly, modern genetics and molecular biology still fail to offer a single piece of experiment or even theoretical clue for an evolutionary "favorable mutation". (Suppl.ExpL.1)

On the other hand, experimental studies for the origin of life were also vigorously performed in modern times. Two early experimental milestones of abiogenesis should be Louis Pasteur's swan flask experiments that challenge the "spontaneous generation" [11] and Miller's experiment [12] that simulated the primitive atmosphere to generate bio ingredients. Later, synthetic biology continuously attracts attention; however, only occasional reports, obstacles similar to above (acquired) genetic modifications of life functions are still obstinately standing. Meanwhile, the origin of life remains a great mystery, for it is more challenging than that of the origin of new (inheritable) traits. Here we will discuss these "origins" from the physical inversion aspects of life and some ancient systems.

\section{"Le Châtelier's effect" and definition of life}

Le Châtelier's Principle states:

When any system at equilibrium for a long period of time is subjected to change in concentration, temperature,

volume, or pressure, the position of equilibrium moves to counteract the change till a new equilibrium is established.

This principle came for in vitro artificially purified few-component individual reactions for more than one century and challenging to be utilized for multi-reaction systems. For a single reaction:

$$
\begin{array}{ll}
\text { suppose } & A+2 B \rightleftharpoons C+D \\
\text { Then } & K c=\frac{[\mathrm{C}][D]}{[A][B]^{2}}(\mathrm{Kc} \text { : concentration constant, } \\
& \text { similarly there are } \left.\mathrm{K}_{\mathrm{P}}, \mathrm{K}_{\mathrm{T}}, \mathrm{K}_{\mathrm{V}} \text {, etc. }\right)
\end{array}
$$

Here constant $\mathrm{K}$ represents the equilibrium (or stationary)

\section{Volume 8 Issue 11, November 2019}




\section{International Journal of Science and Research (IJSR) \\ ISSN: 2319-7064}

ResearchGate Impact Factor (2018): 0.28 | SJIF (2018): 7.426

state under certain environmental conditions; once the condition shifts, constant $\mathrm{K}$ will shift. Not just environmental shift, under the same environmental condition, if products and wastes don't remove out promptly, the constant K could also be shifted. If we put hundreds of single reactions together and wish each one still keeps their original efficiency, one major jinx need to resolve: there should be available one compromised constant $\mathrm{K}$ for all the reactions to assure the efficiencies; also products and wastes need to remove automatically to keep the stability of this constant K. Here "automatic" means all the "removing" processes are driving by synchronized inner physical factors. As in Eq. 2, physical factors such as $K_{P}, K_{T}, K_{V}$, etc., "automatically" steer the chemical factor Kc to follow the principle and also remove the wastes. We can write:

Suppose

$$
\begin{aligned}
& \mathrm{C}_{\mathrm{qi} 1} \mathrm{~K}_{\mathrm{qi} 1}+\mathrm{C}_{\mathrm{qi} 2} \mathrm{~K}_{\mathrm{q} 22}+\ldots . .+\mathrm{C}_{\mathrm{qi}(\mathrm{n})} \mathrm{K}_{\mathrm{qi}(\mathrm{n})}= \\
& \mathrm{C}_{\mathrm{qj} 1} \mathrm{~K}_{\mathrm{qj} 1}+\mathrm{C}_{\mathrm{qj} 2} \mathrm{~K}_{\mathrm{qj} 2}+\ldots . .+\mathrm{C}_{\mathrm{qj}(\mathrm{m})} \mathrm{K}_{\mathrm{qj}(\mathrm{m})}
\end{aligned}
$$

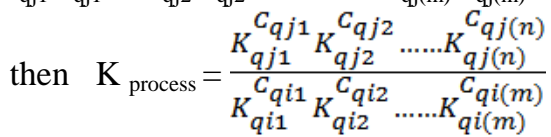

( $i k, j k$ :subscript; $C^{q i k}, C^{q j k}$ :copy number)

(Eq. 3 represents the bundles of single reactions put together. "qi, qj" is the subscript of $\mathrm{K}_{\text {process }}, \mathrm{K}_{\mathrm{qik}}$, and $\mathrm{K}_{\mathrm{qjk}}$ represent constant $\mathrm{K}_{(\mathrm{s})}$ for certain reactions which could express as Eq.2. Human chemical engineering nowadays still can't integrate reactions into such a higher level, and only for bio-systems, we can get Eq.4. The human blood-brain barrier is a good example of the integration of physical and chemical processes together; it is possibly the most advanced chemical and physical integration system on earth.) Due to constant $\mathrm{K}$ is the integration of certain environmental conditions; it needs unifying reaction conditions for functioning; such a unifying condition is in vivo condition. It is why in real-world biological in vivo processes are all multi-reactions with complex matrix ingredients, and a lot of compartments, reticulum, membranes, tissues, etc., are come into reactions. Also, synthesis and removing of products and wastes are synchronized quite well. All these are evolved to resolve the above jinx. In an ideal condition, no intermediate wastes accumulated. Eq. 4 represents an effective equation for an ideal bio-system. (Here the ideal situation means organisms are at a young state. In that state Eq. 4 works well; it only retrogrades due to senility or disease and never from species. For some of the cross-species conditions, such as symbiosis, it is still effective.) This conclusion is not only effective for chemical parameters but also effective for all relevant governing physical parameters. (From the very beginning until today, chemical engineering includes physical parameters such as reaction surface, speed of mixing, etc. Possibly due to the reactions in bio-systems were much more complex to consider physical factors, biologists began to "habitually" forget physics. In a real condition, these two disciplines are inseparable even for non-living beings.) Empirically frog sciatic nerve experiment and Human patellar reflexes demonstrate Le Châtelier's physical effect. These snapshot experiments show Le Châtelier's units. For accumulated or growth Le Châtelier's effects (Suppl.Movie.1.0) and its explanations (Suppl.ExpL.2) gives historical evidence. For those who are not accessible to such ancient systems, (Suppl. Exp. 1-5) offered equivalent modern scientific evidence.

From these verifications, we can define that life is a "Le Châtelier's system", the potential of a living system to issue "Le Châtelier's effect" to its discernable environmental signals is bio-inertia. (The statement "at equilibrium for a long period" mean by the major part of the system has attained to an environmental signal-maximum-entanglement-state, any change will induce the system to maintain the status quo and counteract the change, it can also call "stationary state".)

Peoples in (Suppl. Movie 1.0) got "level one" ancient physical training, thus could resist a much stronger strike than that of untrained people. However, such resistance still can't change the constant $\mathrm{K}$. (A constant $\mathrm{K}$ for a certain process or the entire body is bound with a lot of parameters. As in (Fig.1a), such training can only make a curve B of untrained people becomes curve A, increases uptrend length of the curve and also make retrograde (downtrend) more symmetry with uptrend part.) The uptrend length of the curve is the constant $\mathrm{K}$ stability. That means under similar $\mathrm{k}$ range, trained people can resist outside strikes more powerful than those of untrained people. (Resist means not damage the recovering capacity of the place gets hitting and not induces bruise or any other kind of hurt on the surface or deep tissues, after beating these tissues could still be recovered. Or in other words, no chemical, physical or biological wastes are accumulated; even help remove the old wastes.) More effective binding means the physical strike momentum can be distributed well; therefore, the strikes to a single distributor (such as a cell or a cytoskeleton network) minimized; the people can resist more powerful strikes.

Le Châtelier's effect equals to the Principle of Least Action. The latter remains the center of modern physics; being applied in diverse divisions includes the Theory of Relativity, quantum mechanics, and the string theory. Hamilton's principle is one of its versions; it defines action $\mathrm{S}$ as:

$$
\mathrm{S}=\int_{t 1}^{t 2} L(\boldsymbol{q}, \dot{\boldsymbol{q}}, \boldsymbol{t}) d t \text { then } \delta \mathrm{S}=0,
$$

( $L(\boldsymbol{q}, \dot{\boldsymbol{q}}, \boldsymbol{t})$ : Lagrangian, $\boldsymbol{q}$ : generalized coordinates) (5) From Eq. 5 that "action" should be least, combined with Eq. 4 we then can get:

$\delta K=0$ (or infinitesimal, oscillate nearby 0 with narrow range is defined as "infinitesimal")

$$
\text { or } \begin{aligned}
\Delta\left(\frac{K_{p j 1}^{C_{p j 1}}}{K_{p i 1}^{C_{p i 1}}}\right) \Delta\left(\frac{K_{p j 2}^{C_{p j 2}}}{K_{p i 2}^{C_{p i 2}}}\right) \ldots \ldots \Delta\left(\frac{K_{p j(n)}^{C_{p j(n)}}}{K_{p i(m)}^{C_{p i(m)}}}\right) \geq \Delta K, \\
\left(\text { any item: } \Delta\left(\frac{K_{p j(n)}^{C_{p j(n)}}}{K_{p i(m)}^{C_{p i(m)}}}\right) \geq \Delta K ; 1 \text { to } m, n\right)
\end{aligned}
$$




\section{International Journal of Science and Research (IJSR) \\ ISSN: 2319-7064}

ResearchGate Impact Factor (2018): 0.28 | SJIF (2018): 7.426

a)

Figure 1: Physical definition of bio-in
b)

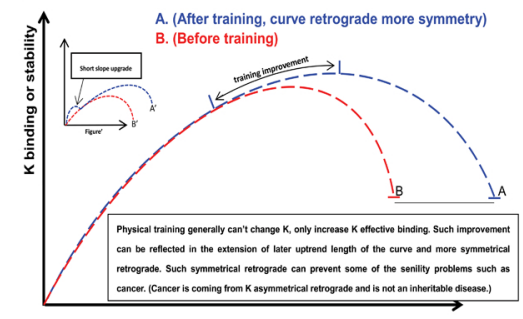

e)

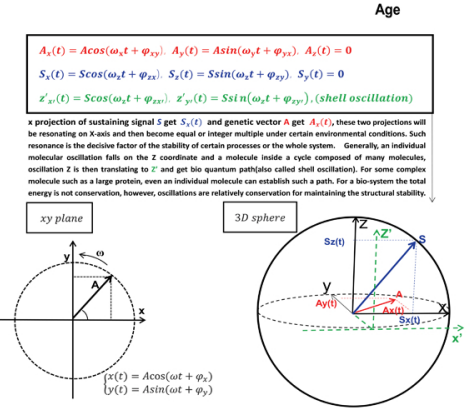

i)

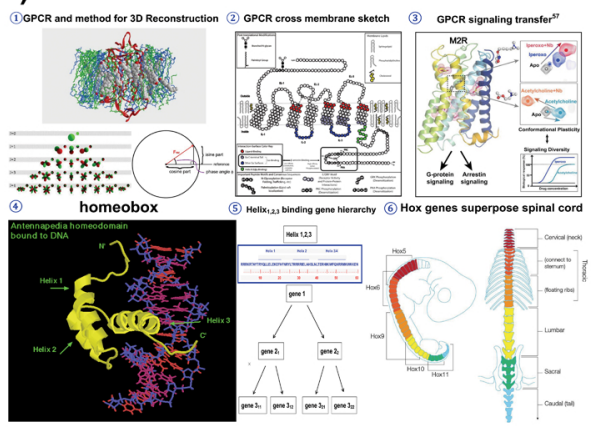

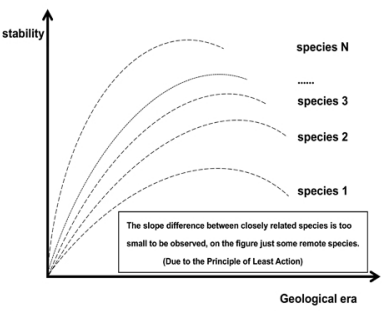

f)
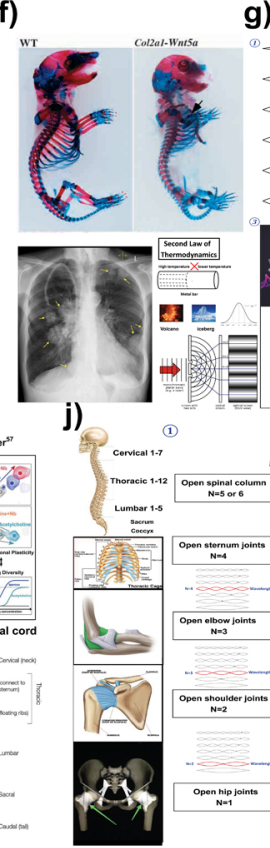

c)

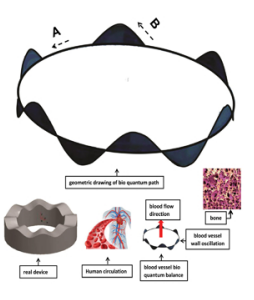

g)

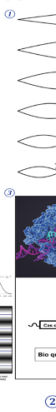

energy inversion quiddity of life

d)

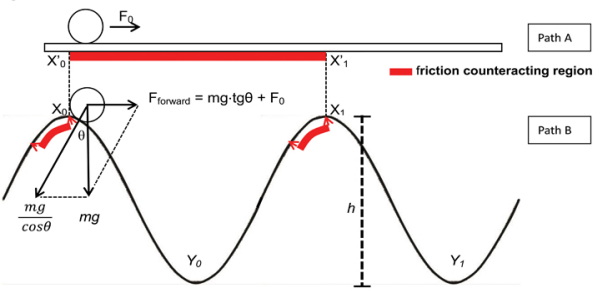

h)
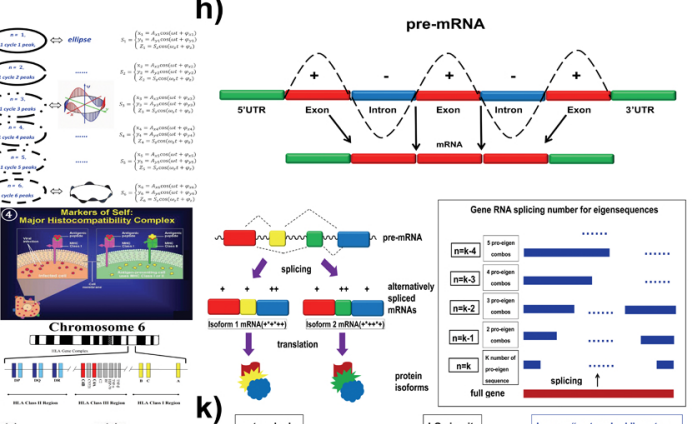

k)

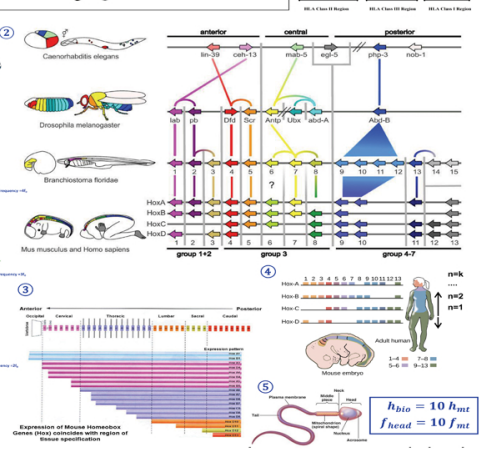

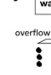
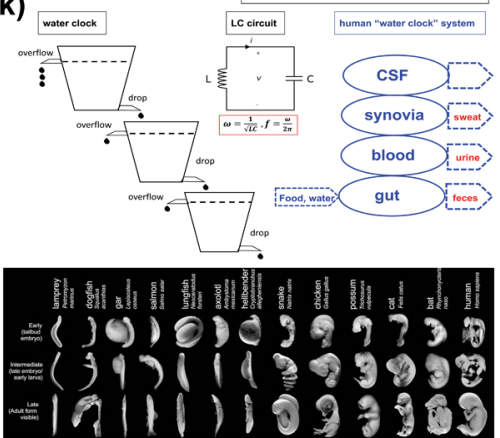

a) constant $\mathrm{K}$ binding shifting curve fit for human ancient physical strike training and other binding parameter modulation

b) constant $\mathrm{K}$ binding shifting curve for diverse species in evolution

c) bio quantum path

d) modulation part of bio quantum path by Newtonian mechanics, the concept of "splicing"

e) mathematical equation of bio quantum path conservative forces (conserve for a larger resonating system which issue this bio quantum path)

f) mobility of normal and cancerous cells, anti-unitary or stationary structure in bio-systems

g) Crisp/Cas 9 and derivative immune systems are self-evolution systems, horizontal \& vertical splicing can be used for modulation surface tension inversion at the genomic level

h) horizontal \& vertical splicing of RNA and gene expression reveals that same gene nucleotide sequence has more than one splicing manner, at least each splicing of a gene has its inversion

conjugated splicing

i) inversion at the cell membrane proteomic level, Hox binding network copy number variation, network inversion

j) ancient CSF-ligament conjugation physical training supplies evidence for modern research on human spinal function

k) water clock model with only one surface tension still can modulate in vivo liquid systems that with multi-surface tension in a certain degree

Eq. 6 or 7 is the biological version of the Principle of Least Action (can also be written as "least squares" version). It means that the younger or higher bio-inertia state of the bio-system, the smaller the difference between the left and right hand part of this equation. As in (Fig.1a) for a time point or a short period of time, the constant K still keeps the same (or changes in an infinitesimal value) while certain internal parameter(s) are changed greatly. (We can roughly estimate the probability of $\delta \mathrm{K}$ shifting, in the binding if only 1 of $n$ parameters is changed for a certain amount, the chance of $\delta \mathrm{K}$ changing is $P_{\delta K}=1 /(n !-1)$, if $n$ is large such probability is quite small. Even if all $n$ parameters have changed, $P_{\delta K}=n /[(n-1) !-n]$ $=1 /[(n-1) !-1]$, still quite small if copy numbers of each parameter are quite lower than the " $n$ ". From here, constant K is a good measurement to follow the Principle of Least Action.) Only in a relatively long period or even whole lifespan, we can then observe the curve of (Fig.1a). The tolerant range of $\delta \mathrm{K}$ change is quite narrow, at the start point and the highest point of (Fig.1a), $\delta \mathrm{K}$ only differ an infinitesimal amount. And break such an infinitesimal amount is enough to make $\mathrm{K}$ turning to retrograde or system malfunction. (Due to binding, this curve is a "smooth discrete curve" and not a continuous curve. From this curve, not only physical strikes but also other interferences such as deliveries of certain genes, stem cells, RNA, etc., are all following the equation. All the parameters are equivalently binding by constant K. Don't misunderstand to think that a gene or any other modern deliveries will be more powerful than those of ancient physical deliveries. All of them are equivalently regulated by the Principle of Least Action, and physical parameters govern chemical or biological parameters. (Technologically, delivered genes on-site are still modified by physical forces continuously. Immunological vaccines can only modify "adaptive immunity" and can never change "innate immunity" or constant K. Even "adaptive immunity" needs a tight $\delta K$ difference for functioning. Senility is a process to divert the $\delta \mathrm{K}$ difference at old ages or $\mathrm{K}$ stability decreased. Therefore, over certain age or health conditions, the efficiency of vaccine interference will greatly be decayed.) Evolution is a similar process that binds constant $\mathrm{K}$ or accumulates binding for Eq.4; just period extended to the geological era. (Fig.1b) refers to holistic constant $\mathrm{K}$ for a series of species. An evolution from one species to another means the slope of the curve is improved. (We only draw a few curves to represent a few species. For how one species can lift 


\section{International Journal of Science and Research (IJSR) \\ ISSN: 2319-7064}

ResearchGate Impact Factor (2018): 0.28 | SJIF (2018): 7.426

constant K slope and evolve to another species we can back to (Fig.1a). Suppose some factors extend the uptrend curve, such as curve B becomes curve A in (Fig.1a). The difference can show as the "training improvement" segment in the figure. This segment not necessarily meant by physical training; any input factors are equally following the same principle. It just means new factor(s) have been binding into curve A and get Le Châtelier's regulation to extend the curve. If this difference is small, then the new binding can't be written into an inheritable carrier. However, while "training improvement" reaches a certain threshold, then the offspring will have a lifted slope of the curve. Such lifted slope is just a short peak, as in the figure' miniature at the up left corner of (Fig.1a). A' is extended from B', and in the start region of A', there is a small "short slope upgrade" peak. (In the figure' all the connections should be smooth curves and the above peak should be very slight; we just exaggerated the peak for viewing.) Only when such a "short slope upgrade" reaches population level and also be extended to the whole curve B' (means the highest point of “short slop upgrade" is near or over the highest point of B' ), then a new species will exist. The whole process is still the Principle of Least Action. Generally, new binding needs a geological time to degenerate into inheritable structures. We can say it is the "least action restriction" that proliferate species.

In physics, a lot of famous equations derived from the Principle of Least Action. It has been regarded as an axiom for centuries that can't be inferred from other physical laws. However, in bio-systems, we can take Le Châtelier's Principle as an axiom and derive the Principle of Least Action: Due to the whole system counteracts to certain changes, then it has to fall into the "least" effect on the system. It is unsure whether Le Châtelier's Principle is more general than that of the Principle of Least Action in our universe. However, it is pretty sure that within bio-systems, it is "father axiom" and the profound causes of the Principle of Least Action. For holistic constant K(s) of a species such as in (Fig.1a), it can bind a lot of parameters. Gene(s) only contribute a part to K. Generally, most of the interferences such as physical strikes, DNA transfer, RNA injections or cell therapy, etc., can't change K ( $\delta \mathrm{K}=0$ or infinitesimal) in short terms. However, once one of the combinations can lift $\mathrm{K}$ "observable value" in the long term by increasing copy numbers or bio quantum path entanglement, all the bindings will be then changed to follow the Principle of Least Action pattern. E.g. the physical training in (Fig.1a), which makes curve B become curve A; will then equally make all other bindings such as DNA, RNA, epigenetic modifications, cells, etc., follow such changes under certain environmental conditions. This time substantial environmental signals have joined the binding to assure previous constant $\mathrm{K}$. This means all the new binding which inheritable must binding environmental parameters. And "symmetrical shift" is vital in preventing senility problems such as cancer. (Modern medical sciences try to find the molecular mechanisms of cancer. However, cancer is generally not an inheritable disease, and most of the molecular reports about cancer are unrepeatable. We still can't get an animal tumor model directly from knocking in or knocking out certain oncogene(s). Embryonic stage interference will induce a lot of deformities, which are still not cancer since they can't metastasis. Go back to Eq. 3 we could know at the embryonic stage, bio-inertia is relatively higher. Following Le Châtelier's regulation, artificially forced knock-in or Knock-out will make the whole system counteract such change(s). Part or all of the bindings will be peeled off functions to maintain the constant $\mathrm{K}$. Such out of tolerating range deprive of functions for diverse bindings will then result in deformities. They are just physical malformations originated from system higher bio-inertia compromise to intolerable man-made interferences and not tumors. In contrast, cancers of distinct subtypes within an organ are usually found to be derived from quite different cells of origin far outside the organ. These prove that cancers are of physical origin, and molecular factors only contribute to a small fraction of the reasons. The real cause of cancer is that bio-systems can't afford two different sets of constant K(s) working at the same time (forked curve in the figure, the real cause of metastasis). The embryonic stage interference induced deformities, no matter how horrifying, are still under the regulation of one set of constant $\mathrm{k}$, therefore are not cancers. Therefore, in (Fig.1a) we state that cancer is induced by the asymmetric detaching of constant $\mathrm{k}$ binding at old age. As in the figure, constant $\mathrm{K}$ detachment at old age is an unavoidable process, and "asymmetric detaching" will easily induce two or more different constant $\mathrm{K}(\mathrm{s})$ work simultaneously at a downtrend curve. The only way to deal with cancer is to make a constant $\mathrm{K}$ detaching processes become more symmetric. (Such symmetry at least means the parameter detaching sequence equals the parameter binding sequence.) For symmetric retrograde of constant K, a proper ancient physical training system is veritably the simplest and the most effective way in the human history of all time.

From the physical definition of life, here we need to emphasize that life is never endowed by the things we usually see, such as DNA, RNA, and cells, etc. It comes from the recovering capacity or the constant $\mathrm{K}$ bindings of the abovementioned structures instead of the structures themselves. (E.g. While an outside DNA sequence inserted into a genome, the whole genome or at least the active region correlated with this sequence will then counteract its change. After counteracting the change made by this sequence to the whole genome to a minimum or equivalently, its distribution to the whole genome attains the maximum, then it is activating. It is such a responsive recovering capacity or Le Châtelier's effect of it that indeed endows life instead of any sequence. Once such responsive recovering capacity is diminished to some degree by senility or other reasons, life will end no matter what kind of sequences are residing on the genome. It is recovering capacity governs gene functions and not in a reverse way, such as gene sequences can fully decide functions, etc. Not only DNA, all other known biostructures \& bio units such as cells, tissues, etc., are governed by this law. We can call it the recovering law of bio-systems.) We emphasize "recovering capacity" just to remind it is the fundamental difference between living and non-living beings. Even under the genetics or molecular biology point of view, there is no big difference between the DNA of a dead and a living being, or the sequencing result is the same; their constant $\mathrm{K}(\mathrm{s})$ are absolutely different. The DNA of a corpse has no constant K, and that of a living being possesses diverse constant $\mathrm{K}(\mathrm{s})$ at different DNA structural levels, locally and holistically. To understand such a difference is the fundamental tasks of

\section{Volume 8 Issue 11, November 2019}




\section{International Journal of Science and Research (IJSR) \\ ISSN: 2319-7064}

ResearchGate Impact Factor (2018): 0.28 $\mid$ SJIF (2018): 7.426

genetics or molecular biology, albeit it is challenging.

\section{Binding of gravity}

Newtonian law of universal gravitation is:

$$
F=G \frac{m M}{r^{2}} \quad\left(\mathrm{G}=6.674 \times 10^{-11} \cdot \mathrm{m}^{3} \cdot \mathrm{kg}^{-1} \cdot \mathrm{s}^{-2}\right)
$$

Einstein's modifications can be understood by calculation of the curvatures of a photon, an apple $(100 \mathrm{~g}$, diameter $=10 \mathrm{~cm})$ and the moon $\left(\mathrm{M}=7.3477 \times 10^{22} \mathrm{~kg}, \mathrm{r}=1,737.10 \mathrm{~km}\right)$ :

$$
\text { Curvature }_{\text {photon }}=\frac{E}{r^{2} c^{2}}=\frac{h v}{r^{2} c^{2}}
$$

(Planck constant $\mathrm{h}=6.626 \times 10^{-34} \mathrm{~J} \cdot \mathrm{s} ; \mathrm{v}, \mathrm{c}$ )

$$
\text { Curvature }_{\text {apple }}=G \frac{M_{\text {earth }} m_{\text {apple }}}{r^{2}}=\text { constant } \cdot \frac{m_{\text {apple }}}{r^{2}}
$$

For Eq. 9 we roughly take $r=$ wavelength $\lambda$, (the wavelength of visible light is $400-760 \mathrm{~nm}$ ), the result is around $10^{-22}$, this value is so small to impact nearby space-time, we can then get the first principle for theory of relative: The speed of light in a vacuum was independent of the motion of all observers. For Eq.10, $\mathrm{G}$ and $\mathrm{M}_{\text {earth }}$ are fixed number, $\mathrm{K}=\mathrm{G} \cdot \mathrm{M}_{\text {earth }}$, then:

Curvature $_{\text {apple }}=\mathrm{K} \cdot \mathrm{m}_{\text {apple }} / \mathrm{r}^{2}=\mathrm{K} \cdot 10 \mathrm{~kg} \cdot \mathrm{m}^{-2}$, similarly, Curvature $_{\text {moon }}=\mathrm{K} \cdot 2.4 \times 10^{12} \mathrm{~kg} \cdot \mathrm{m}^{-2}$

The curvature induced by an apple and even by the moon is not enough to change the results of the application of the Newtonian system on Earth due to the lower mass distribution density. Therefore, the denotation of (8) is Newtonian mechanics, the denotation of (9) and (10) is Einstein's Theory of Relativity. However, (Suppl.Exp.6-8) demonstrate that the gravity of living organisms are quite different from the value when it is dead, the range is $0.14 \%-3.8 \%$, which is challenging to be explained by the above two systems. (In Cavendish or later modified methods for non-living being $G$ measurement, the affinity forces on the device are only $10^{-11}$ of the weight of the attracting balls. Therefore, it can only perform by world-famous experimental physicists with highly sensitive Cavendish torsion balance. However, once such differences increased from $10^{-11}$ to $0.14 \%-3.8 \%$ of the measuring weight in bio-systems, that means gravity enlarged to $10^{9}$ (median calculation). Then just a common people with a cheap balance and lower level experimental skills can perform quite well, no need to consider some error factors such as air buoyancy and air disturbance, etc.) In Eq. 8, M, m, and r remain almost the same, therefore such "gravitational loss" should come from the shifting of G. Einstein has developed his field equation to modulate the G to: $\kappa=\frac{8 \pi}{C^{4}} G$, however, this way still far beyond so large discrepancy on same existence with same space curvature and lower speed conditions can include. If $\mathrm{G}$ of nonliving things are correctly described by the Newtonian and Einstein's theories on the different empirical conditions, the $\mathrm{G}$ shifting in bio-system should come from the constant $\mathrm{K}$ binding of gravity, Eq. 8 can then be modified as:

$$
F=K^{N} \cdot G \frac{m M}{r^{2}} \quad(\mathrm{~N} \text { : copy number })
$$

Eq. 11 can only be used for estimating the gravity shifting between a living being and Earth; it can't be used for measuring the affinity between two living beings since the inertia mass is quite different from the gravitational mass and has fallen into complex non-linear relationships. For nonliving beings, inertia mass equals to gravitational mass is the foundation of modern physics. It has been "validated" by Eötvös and other experiments, and till today at $10^{-12}$ precision, these two masses are identical. However, the Eötvös experiment and free-fall verification have a hidden premise to get conventional results. Once the hidden premise is broken, even for non-living beings, the result will tend to the bio-system conclusion (Suppl. ExpL. 3). The most convenient way to verify inertia mass VS gravitational mass relationships is still the derivative designs of (Suppl.Exp.6-7). The significance of Eq. 11 is not just for such validation; we possibly can monitor in vivo gravitational waves for real biological applications. From Eq. 11, whether a transferred gene inside an animal is inheritable will be decided by the gravitational loss of the whole body it induced. (While previously referring to "training improvement" in (Fig.1a), we roughly say when that extended curve reaches a certain level, it could be inheritable. Now we can say that "gravitational loss induced by it to the whole system reaches a certain threshold", then it can be inheritable.)

Another concept "time dilation" (a difference in the elapsed time measured by two observers) is also from Einstein's Theory of Special Relativity: $T=T_{0} /\left(1-V^{2} / C^{2}\right)^{1 / 2}(C$, speed of light; V, speed of an object). Some people are always misunderstanding to regard this equation as proof that the highest speed in our universe is from light; nothing can exceed this speed. Logically no theory with human knowledge can be qualified for such kind of "proof". It only says that "the speed of light in the vacuum is independent of observers"; therefore, it can utilize such "independent speed" to establish a reference system. (In the future, if there is a speed higher than that of light could be presented, just some modifications following the Principle of Relative are enough since the speed of light is yet the most reliable reference at the present technological level.) However, light speed reference is only effective for a system that Le Châtelier's effect not present or can neglect. (Michelson-Morley Experiment and the way modern physics deals with this experiment can be interpreted as there is no Le Châtelier's effect to be taken into account while a beam of light passes a space. Any changes made by this beam of light pass through space can be independently measured by all the parameters induced by it, and all of them are absolutely "measurable". However, in bio-systems due to Le Châtelier's constant K binding or Eq.4, the speed of light can't simultaneously act as references for all commutating parameters. Once it can refer well for some parameters induced by it, then it will definitely refer to system enlarged errors for other commutating parameters. This means that "observation" or "observers" are no longer absolutely "available". From the motivation that the Theory of Relative tries to create an unbiased reference for all observers, it can work well for an observable system and will become reluctance once all "observers" are not equally available.) However, in the latter condition we still can estimate time dilation in bio-systems as:

$$
T=\frac{T_{0}}{\sqrt{1-\frac{R^{2}}{R_{0}^{2}}}}=\frac{T_{0}}{\sqrt{1-\frac{A^{2}}{A_{0}^{2}}}}
$$

$\mathrm{R}$ and $\mathrm{R}_{0}$ are "recovering radii" for which Le Châtelier's effect can be presented to certain degrees. A and $\mathrm{A}_{0}$ are "oscillation amplitudes" which we'll refer to later. In bio-systems, there is

\section{Volume 8 Issue 11, November 2019}




\section{International Journal of Science and Research (IJSR) \\ ISSN: 2319-7064}

ResearchGate Impact Factor (2018): 0.28 | SJIF (2018): 7.426

no time dilation; only time inversion or time commutation exists, it is equivalent to time dilation concept under lower Le Châtelier's effect or high speed condition. Beyond a series of Le Châtelier's radii or oscillation amplitude, the system will be retrograded into nonliving systems.

\section{Binding of "conservative forces" and the sustaining of bio quantum path}

Time and space in a Newtonian system "never concern with anything inside space". In Einstein's system, time and space began to concern with each other and with mass distribution. Bio curved space is different from the above two models, we can discuss from an experiment:

In the geometric diagram of (Fig.1c), path $\mathrm{A}$ is the circumference of a circle; path B is a wave-shaped curve along the same circumference (bottom left is a real device; here not show the side rail or concave for holding a ball running inside it). One ball with the same energy run through path A and path $\mathrm{B}$, which way could be faster and save time? Common sense believes that path $\mathrm{B}$ needs to go through many crests and troughs or includes much longer running distance than that of path A, with the same energy path A should save time. However, we can watch (Suppl. Movie 3), path B does save time than path A. (This design actually comes from a mathematical problem in Newtonian times known as "brachistochrone curve" which put forward by Johann Bernoulli. At that time, many famous people included Newton and Leibniz had joined the challenge and got the results. Bernoulli himself took the Fermat Principle which was the earliest version of the Principle of Least Action for a solution. Now we connect many brachistochrone curves to get a wave-shaped curve as path B. It is actually not limited to brachistochrone curves, any kind of smooth curve will work to save time for this physical experiment. Just those composed of brachistochrone curve segments can save a little bit more time than other shapes.) (Fig.1d) is the analysis of the difference between path $\mathrm{A}$ and path $\mathrm{B}$ by Newtonian mechanics, if we offer a same horizontal slight $\mathrm{F}_{0}\left(\mathrm{~F}_{0}\right.$ can't be large enough to make ball jump on path $\mathrm{B}$ ) to the balls on path $\mathrm{A}$ and path $\mathrm{B}$. The ball on the path $\mathrm{A}$ has to depend on this ephemeral promotion $\mathrm{F}_{0}$ to move forward. However, for the ball on path $\mathrm{B}$, besides this same promotion $\mathrm{F}_{0}$, it still gets a force $m g \cdot \operatorname{tg} \theta$ comes from gravity to drive the ball moves forward: $d F_{\text {forward }}=F_{0}+m g \cdot d \operatorname{tg} \theta$. (In the figure, $\theta$ is the angle between the perpendicular line from the ball to its contact surface and vertical gravity. This angle starts from $0^{\circ}$ at the highest point $\mathrm{X}_{0}$ and gradually increases to the maximum and then gradually back to $0^{\circ}$ at the lowest point $\mathrm{Y}_{0}$.) While the ball falls down to $\mathrm{Y}_{0}$ it has stored gravitational potential energy $\mathrm{mg} \cdot \mathrm{h}$ and can go back to the next highest point $\mathrm{X}_{1}$ if there is no energy loss such as frictions. This process continuously recovers and then drives the ball on path $\mathrm{B}$. If we consider friction, the region in which friction slowing down the movement of the ball can be defined as the "friction counteracting region". In the (Fig.1d) for one period, suppose the corresponding points of path $\mathrm{A} X_{0}^{\prime}$ and $X_{1}^{\prime}$ equivalent to path B's high points: $X_{0}$ and $X_{1}$. Then the entire distance from $X_{0}^{\prime}$ to $X_{1}^{\prime}$ on path A are "friction counteracting region" marked by red color. However, the "friction regions" on path $\mathrm{B}$ are the only small red color region in front of the $X_{0}, X_{1}$. Therefore for path $A$ the promotion force $F_{0}$ (or primary momentum) needs to overcome the frictions in the whole path A. $\mathrm{F}_{0}$ (or primary momentum) on path $\mathrm{B}$ only needs to overcome the frictions in the small red color region due to the help of gravity. This is why we can directly watch a ball on path $B$ is running faster and saving time than the one on path $A$ in the movie. (In physics, gravity is a "conservative force" while friction is a non-conservative force. A conservative force can be defined as $W \equiv \oint_{C} \vec{F} \cdot d \cdot \vec{r}=0$, which the total work done in moving a particle between two points is independent of the taken path.) Life function or evolution can depend on path $\mathrm{B}$ or its derivatives to strengthen conservative forces and weakening non-conservative forces and then maintaining the system; we call this process "splicing".

For the shape of curve B, it can be diverse wave-like shapes just symmetrical can then assure the above process. In physics, a simple harmonic motion is a type of periodic motion or oscillation motion where the restoring force is directly proportional to the displacement and acts in the direction opposite to that of displacement. This path can be then projecting on coordinates and get a simple harmonic motion on each dimension of $\mathrm{x}, \mathrm{y}, \mathrm{z}$ under ideal conditions. (Left of (Fig.1e) demonstrates how a uniform circular motion vector $\vec{A}$ projecting on $\mathrm{x}, \mathrm{y}$-plane to get simple harmonic motion $\mathrm{A}_{\mathrm{x}}(\mathrm{t})$ and $\mathrm{A}_{\mathrm{y}}(\mathrm{t})$. If an orthogonal $\mathrm{z}$-dimensional simple harmonic motion $\mathrm{S}_{\mathrm{z}}(\mathrm{t})$ is resonating into then can get path $\mathrm{B}$. Right of (Fig.1e) demonstrates how this happens in a 3D sphere. Resonating here means the projections of two rotation vectors on the same axis become equal: $A_{x}(t)=S_{x}(t)$.) The environmental signal which keeps the same vector independent of system movement and is also discernible by the system is defined as a sustaining signal. (Like the gravity $\mathrm{m} \cdot \overrightarrow{\mathrm{g}}$ in the above case, keeps the same vector no matter how the ball is moving in the system scale. Here "discern" means Le Châtelier's effect can be happening to splice out non-conservative "resistance".) In (Fig.1e) Z dimension is the sustaining signal direction. (We use the terminology "signal" to replace "force", just due to constant $\mathrm{K}$ binding is more general than "force" in issuing Le Châtelier's effect.) Then path B can be defined as a "bio quantum path". (The primary sustaining signal on Earth is only gravity. A series of "bio quantum paths" share the same sustaining signal then evolved into earlier life. Later, other parameters such as sunlight, water pressure, etc., join in and then create many derivative sustaining signals. With the progressing of evolution, all the in vivo movements refer to a certain part (or the whole system) that independent of the movements of this part (or the whole system) are sustaining signals. Like a blood flow in the bottom of (Fig.1c), every such region of a blood vessel can be modulating by it. The quiddity of bio quantum path is the superposition of a very general strengthening \& weakening effect. As in the figure, path B can splice out friction by strengthening \& weakening effect sustaining by gravity, and path A can't.) Bio quantum path can project as:

$$
\left\{\begin{array}{l}
x=A_{x} \cos \left(\omega t+\varphi_{x}\right) \\
y=A_{y} \cos \left(\omega t+\varphi_{y}\right) \\
z=S_{z} \cos \left(\omega_{z} t+\varphi_{z}\right)
\end{array}\right.
$$




\section{International Journal of Science and Research (IJSR) \\ ISSN: 2319-7064}

ResearchGate Impact Factor (2018): 0.28 | SJIF (2018): 7.426

Mathematically Eq. 13 is an ellipsoid in which every section is an ellipse or circle if $\omega=\omega_{z}$, no matter how A, S, and $\varphi$ are changing. (This is the reason why eggs are ellipsoid in shape since bio-inertia are maxima. With development, $\omega_{\mathrm{z}}$ gradually different from $\omega$, we call this converge which we'll refer to later. ) (Suppl.Exp.9-12) are some general experiments for it.

We define the "bio quantum path" instead of "bio force" for the above model for a few reasons. First is that not only physical forces but also any other signals (memory is a kind of signal transferring, it follows the same Eq. 13 with the forces we move our hands \& legs just with different "splicing" number.) fall into above "path" (partially or totally) will get an equivalent effect: "recovering capability". (A Newtonian force that has been described by his three laws of motion, can be written as a vector $\vec{F}$. The "recovering capability" of such a vector $\vec{F}$ is quite lower. If it is superimposed with any other vector force $\overrightarrow{F^{\prime}}$, it will easily be changed either on direction or quantity or both direction and quantity by parallelogram law. However, for a resonating force on a bio quantum path, the superposition result of it with a vector force is still a bio quantum path (refer to Fig.1d). Sometimes there is even no detectable shift in $A, \omega$ as in Eq. 13 since the vector forces are not just superposed with bio quantum path we see; it actually needs to superpose with large resonating structures that issuing the bio quantum paths. If a resonating force meets with another resonated force or a wave series, the result still keeps a bio quantum path pattern even if something such as interference or diffraction is happening. "Keeps the similar pattern" means the "recovering capability" of the resonating force or signal is issuing.) Now we can understand that for offspring, they not only need biomaterials from parents but also need these inherited materials to possess enough bio-inertia or oscillation frequencies. The swimming of spermatozoa in the biological kingdom is due to the physical oscillation requirement of reproduction processes. The second reason, we can see some properties of forces or signals which have integrated on bio quantum paths. Newtonian vector forces are usually separated from inertia. $\vec{F}=\mathrm{m} \cdot \mathrm{d} \vec{v} / \mathrm{dt}$, $\mathrm{m}$ decide inertia and $\vec{F}$ can't influence on inertia without acting on a mass. (For something like a photon which is massless, vector force generally can't influence inertia.) However, in bio-systems, all the mass are normalized, a big cell or a small cell, or even a tissue possesses the same normalized mass; therefore, in vivo momentum can be issuing by force without the involvement of a mass. (The Newtonian mass concept has been replaced by a "structural concept" (bio quantum path) in bio-systems. Like Newtonian forces can't be separated from mass to issue works, "bio forces" still can't be separated from the structure or bio quantum path to issue functions.) Third, we also note that the "conservative forces" or signals in bio-systems are slightly different from that in physics. In physics, conservative forces only refer to the path VS work done and seems never involved with mass or can be separated from the mass. In bio-systems, conservative forces become sustaining signals, bio quantum paths (structures) and can no longer be separated. This is why bio-systems can fix so much gravity. We should also understand that no matter Newtonian or Einstein's theory, gravity is quite difficult to make a bundle of objects to demonstrate Le Châtelier's correlation. However, once gravity is bound into bio quantum paths in vivo, each structure is then unexceptionally endowed with Le Châtelier's effect. Therefore, in physics, gravity is a conservative force without any Le Châtelier's effect. In bio-systems, it is the origin of conservative forces (bio quantum paths) and Le Châtelier's effects. Primary sustaining signal or "conservative force" gravity on Earth always takes roughly the same vertical direction; however, "secondary" sustaining signal or "conservative forces" in bio-systems can shift to diverse other courses. Also, biomaterials running on it will not unlimitedly "independent of the path taken" and always leaves some infinitesimal amount; with senility the "splicing out resistance capability" or tolerance for "infinitesimal leftover" from bio quantum paths will gradually decrease; however, still follow the principle of least action to get a lower $\delta \mathrm{k}$ even while losing binding. Life in generation can't be eternal just due to this reason, "splicing" can only splice out resistance to the infinitesimal amount instead of "0".

However, on the other hand, bio quantum still path can be called "bio perpetual motion path". "Perpetual motion machine of the second kind" seeks to continuously acquire energy from certain sources. Since this path is conceptually and realistically most close to the motivation of the "perpetual motion machine of the second kind" and life does depend on it for collecting and transforming sustaining signal, we can call it "bio perpetual motion path". (In thermodynamics, Clausius Inequality is written as $\oint \frac{\delta Q}{T} \leq 0$, only for a reversible condition $\oint \frac{\delta Q}{T}=0$. Some people then take this inequality to deny the possibility of perpetual motion since among all the designs, the irreversible processes are unavoidable. However, this inequality is acquired from an isolated system. Biosystems are actually open systems. All the living beings we see are real natural "perpetual motion systems". Generation to generation, biosphere can be regarded as "eternal"; therefore, eternal life concept not necessarily depends on " $\oint \frac{\delta Q}{T}=0$ ", alternative " $\oint \frac{\delta Q}{T}=$ infinitesmal" still works well.) In the (Suppl. Movie 3) experiment, due to the procuring of a sustaining signal, the ball runs on a curved path B will save time and runs faster than that runs on a Euclidean geometric shortest path A. This is actually the concept of curved space. And such bio "curved space" is different from that of mass distribution curvature and energy space. It depends on "sustaining signal" for "curved" and "splicing" characteristics. (Einstein ring or Einstein lensing effect is an astronomical phenomenon while light passes through a large celestial body will be bent. The angle of the bent is: $\theta=\frac{4 G M}{r c^{2}}=2 \frac{r_{s}}{r}, \mathrm{G}$, $\mathrm{M}$, c, and $\mathrm{r}_{\mathrm{s}}$ are Newtonian gravitational constant, mass, the speed of light, and Schwarzschild radius. It was published by 1936 and till 1980 got first verification. Now, if we take the light pass through the edge of an animal body or observe the cells under a microscope, we still can visualize a similar effect by adjusting the angle and strength of light. However, such an effect in bio-systems is not induced by mass distribution. The $\mathrm{z}$ dimensional signals from diverse bio quantum paths contribute substantially. This is why bio curved space which upholds by sustaining signals, is different from Einstein's curved space

\section{Volume 8 Issue 11, November 2019}




\section{International Journal of Science and Research (IJSR) \\ ISSN: 2319-7064}

ResearchGate Impact Factor (2018): 0.28 | SJIF (2018): 7.426

that upholds by mass distribution. In Einstein's curved space, gravity can be neutralized. In a bio curved space, all biological functions are based on $10^{9}$ levels of gravitational binding, quite difficult to be neutralized unless the system is damaged.)

In physics and other disciplines, the law of conservation of energy is generally accepted for an isolated system or "isolable research targets". However, bio-systems are non-isolated systems; this law becomes reluctance. Back to (Fig. 1d) we can see, path A is a conventional energy conservation model which agreed by all systems, running a ball on a plane, the primary momentum $m v_{0}$ is gradually damping down by friction over time t. Diverse physical equations can be written down for such conservation. Empirically, once we make a path and a ball uniformly and offer a fixed $\mathrm{F}_{0}$ (the small slant in Suppl. Movie 3 is for this purpose); in each repetitive running, the ball will finally stop on a region with narrow shifting; that means energy conservative. And the theoretical calculation is easy and will be not far away from the experimental result. However, for path $\mathrm{B}$, things become complicated. If the system is regarded as an isolated system such as using a black box to cover path A or path B, energy is no longer conservative due to the participation of gravity outside the system. It is challenging to use known parameters such as $\mathrm{F}_{0}$, friction, the mass of the ball, and shape of the bio quantum path, etc., to theoretically predict how far away a ball will run on path $B$, we have to rely on actual measurement. Experimentally, even if we can make everything uniformly, the stop distance of the ball will shift widely for each running. (A ball on path B generally can run more than 50 times of the vertical distance as that on path A with the same materials and contact surface. On path A, the final stop distances will within $5 \%$ of the average in a group of 30 repeats. On path B, 200-300\% of the average running distance will usually happen in the same 30 repeats. If energy is conservative, once parameters fixed, the ball on path B should stop at a narrow region like that on path A, only non-conservative of energy due to the participating of gravity can get such wide random shifting. This means path $\mathrm{B}$ is more easily impacted by environmental oscillations than that of path A due to the participation of gravity. And such kind of environmental oscillations can't be eliminated by vacuum; for any system, vacuum $\times$ non-vacuum $=k$.) Here the case just one bio quantum path, in bio-systems, all the forces and signals can only be transferred by diverse bio quantum paths. Energy conservative discrepancy will be more significant than in the figure. We can define that resonation energy that can be adapted into or transferred by Eq. 13 as "conservative energy", no matter which mechanism is involved. The indiscriminate energy we usually see is non-conservative energy that can't be utilized by bio-systems. (This is why we can't feed animal electricity or petroleum to produce meat for us. It estimated for advanced species like human beings; the energy "splicing" number has been over 1 million; that means from every 1 million units of energy, we can only utilize 1 unit of "conservative energy". In bio-systems, "conservative energy" is still relatively conservative in a certain period; just the indiscriminate energy is not conservative.) To further understand energy conservative and the role of gravity in evolution, we can go to Einstein's field equations of general relativity [14][15]: $R_{\mu \nu}-\frac{1}{2} R g_{\mu \nu}=\frac{8 \pi G}{c^{4}} T_{\mu \nu}$, Newtonian $\mathrm{G}$ is the term with $\kappa=\frac{8 \pi G}{c^{4}}$, to simplify into Nonrelativistic
Newtonian G need to write stress-energy tensor in a field of matter with low proper density $\rho$ and low velocity $\mathrm{v}$ as [16]:

$$
T_{\mu \nu}=\rho\left(\begin{array}{cccc}
1 & \frac{v_{x}}{c} & \frac{v_{y}}{c} & \frac{v_{z}}{c} \\
\frac{v_{x}{ }^{2}}{c^{2}} & \frac{v_{x}{ }^{2}}{c^{2}} & \frac{v_{x} v_{y}}{c^{2}} & \frac{v_{x} v_{z}}{c^{2}} \\
\frac{v_{y}}{c} & \frac{v_{y} v_{x}}{c^{2}} & \frac{v_{y}^{2}}{c^{2}} & \frac{v_{y} v_{z}}{c^{2}} \\
\frac{v_{z}}{c} & \frac{v_{z} v_{x}}{c^{2}} & \frac{v_{z} v_{y}}{c^{2}} & \frac{v_{z}^{2}}{c^{2}}
\end{array}\right)
$$

For bio-systems based on board facts and the gravity binding quiddity, bio-evolution is actually the normalization of the time dimension. We add oscillation at time dimension into (13) then get a general equation for all bio-systems:

$$
\left\{\begin{array}{c}
x=A_{x} \cos \left(\omega t+\varphi_{x}\right) \\
y=A_{y} \cos \left(\omega t+\varphi_{y}\right) \\
z=S_{z} \cos \left(\omega t+\varphi_{z}\right) \\
t=A_{t} \cos \left(\omega t+\varphi_{t}\right)
\end{array}\right.
$$

For the existing of Eq. 15, developmental inversion [13] offers enough evidence. (Actually, just from (Fig.1d) we could know that there are commutation relations inside the bio quantum path model. $\mathrm{Z}$ dimension has persisted priority over that of $\mathrm{x}, \mathrm{y}$, and $\mathrm{x}, \mathrm{y}$ have conditional commutations with each other. Here time commutation just means it can be written down as $t=A_{t} \cos \left(\omega t+\varphi_{t}\right)$.) Such kind of commutations will be inverted by Le Châtelier's effect to make t exchanges with $\mathrm{x}, \mathrm{y}$, $\mathrm{z}$ dimensions under certain conditions, that is an inversion. (Sexual behavior is a special type of inversion, just $\mathrm{z}$ dimension inversed with that of $\mathrm{x}, \mathrm{y}$ to lose part of time-dimensional binding or bio-inertia. Refer to (Suppl. Exp.13) for empirical evidence. Later we'll further refer to the inversion process.) All dimensions of Eq. 15 can be mathematically combined into one locus equation (with roughly the same phase angle $\omega$ ), with coefficient matrix and constant term D as:

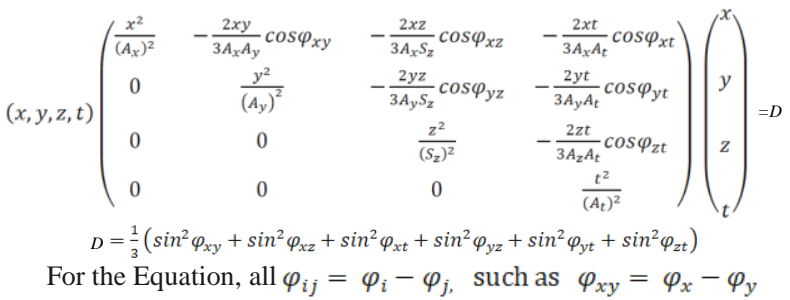

Following Eq. 14, Einstein's stress-energy tensor which deals with non-living systems is symmetric, according to Noether's theorem, its energy is conservative; however, in bio-systems, the coefficient matrix in Eq. 16 is not symmetric; according to the theorem, energy is not conservative. This means that bio-systems need continuous input for maintenance; such inputs are conservative energies mainly compensated for $\mathrm{z}$ dimensional oscillation. (From these discussions we could understand; for quantum mechanics which relies on simple harmonic motions, time still should be commutation time $t=A_{t} \cos \left(\omega t+\varphi_{t}\right)$. This discipline made the commutation time into the unitary time, then can be fit into the energy conservative format we usually see, and also avoided the inversion processes.) If we don't consider the time dimension and only take bio quantum path from Eq. 13, we still can combine dimensions to get the coefficient matrix and constant

\section{Volume 8 Issue 11, November 2019}




\section{International Journal of Science and Research (IJSR)}

ISSN: 2319-7064

ResearchGate Impact Factor (2018): 0.28 | SJIF (2018): 7.426

term D as follow:

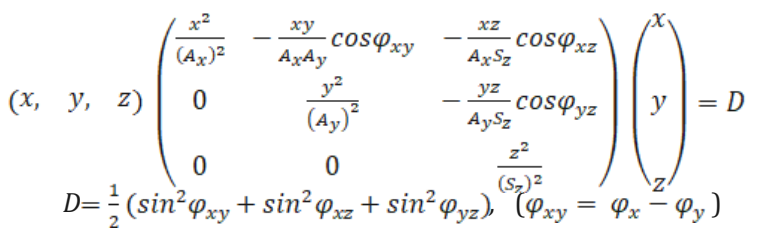

Following Noether's theorem, the energy conservative conclusion keeps the same. (As in a bio quantum path, if $\mathrm{z}$ dimensional input is enough and can be resonating with other dimensions well, the system is in an active state and can splicing out resistance quite well. However, once the input in this dimension is decaying to a certain degree, the system has to transfer part of $\mathrm{x}, \mathrm{y}$ dimension to $\mathrm{z}$ for maintaining, such "stress condition" can't last long since the splicing capability is greatly reduced.)

The trace of the coefficient matrix in Eq. 14 or Eq.16, which is the sum of the diagonal elements from top left to bottom right, is equal to zero for potential fields (Laplace's Equation), or following the Principle of Least Action they both should tend to "least action"; therefore, equivalent in a fixed time period. Compare the trace of $T_{\mu \nu}$ and the coefficient matrix of Eq. 16, we can get:

$$
A_{t}=t=A_{x}=A_{y}=S_{z}=c
$$

Eq. 17 just means in bio-systems, we can't use c as a reference; the reference significance of $\mathrm{c}$ becomes oscillation amplitude (ref. Eq.12). It is not difficult to understand that bio-systems take oscillation amplitude as reference for gravitational effect, and nonliving beings in astronomical scales take $\mathrm{c}$ as reference for gravitational effect.

The above discussions that "bio-systems functioning by bio quantum path gravitational binding" are achieved through scientific research approach. From the technical approach, we still have quite stronger evidence. A system known as HiGee (High Gravity Rotary Device) or RPB (Rotating Packed Bed) has been developed since the late 1970s (Suppl. Ani 1). It involves the application of high gravity fields, typically $500-1000 \mathrm{~g}$, in order to intensify or manipulation the relative mobility, mixing rate or heat/carried particles transferring efficiency, etc., among two-phase or multiphase; for industrial applications such as HiGee distillation, seawater de-aeration, nano-synthesis, selective absorption $\left(\mathrm{H}_{2} \mathrm{~S}\right)$, reactive stripping of compounds (Vitamin B12), waste gas removal $\left(\mathrm{SO}_{2}\right)$, etc. The relative mobility between two-phase can directly modulate from Archimedes' principle:

Relative mobility $=$ buoyant factor $=($ density of liquid $)$ (gravitational acceleration)(volume of liquid)

$\rightarrow R_{\text {mobility }}=\rho g V=\rho g h v=\rho g R_{h v}$ (h: horizontal scale of the liquid, v: vertical scale of the liquid)

Neglect the tiny shifting of $\rho, \mathrm{V}$, and regard them as constant, while $\mathrm{g} \rightarrow 0$, then $\rho \mathrm{gV} \rightarrow 0$; this trend means the relative mobility tends to zero with the $\mathrm{g}$ tends to zero. At this state, molecular surface tension and its derivative forces will govern the system; therefore, diverse molecular agglomerations will tend to a minimum surface or maximum folding state; separation tends to a more difficult condition. In contrast, the more increase of $\mathrm{g}$, the more increase of relative mobility; and now shear stress (average shear stress $\tau=F / A ; \tau$, shear stress, $\mathrm{F}$, the force applied, A, the cross-sectional area of material with area parallel to the applied force vector.) not only overcomes the surface tension and increases the relative mobility between phases but also stretches the liquid to "liquid membrane" and then greatly increases the contact surface area, this simultaneously increases the exchanging of inner phase carried stuff such as heat/carried particle (affinities), etc. (The increasing of phase relative mobility means two phases leave quickly and increasing the exchanging of inner phase carried stuff are two processes that always combined together in most conditions, albeit these two processes usually make people believe that they should happen in a contrary way. The increasing of gravity can bind these two seemingly contrary processes.) This induces the phase separation or increasing the phase inner carried stuff exchanging rate become easier; diverse molecular agglomerations will then tend to a maximum stretching state instead of a folding state. The above theory has been developed to explain HiGee for decades, due to HiGee is only industrially applied for a non-living system till now, we need to modify its theory for adapting in bio-systems. From the technological details in (Suppl. ExpL. 4), surface tension $\gamma \propto \mathrm{g}$, we can write $\gamma=\mathrm{g} \bullet \mathrm{K}$; here $\mathrm{K}$ is the constant $\mathrm{K}$, in mixed substance or multi-phase conditions, even if environmental parameters are fixed, this $\mathrm{k}$ will be in a certain range; $K=\gamma / g$ will be shift diversely for non-living beings; for living beings, due to evolution $K=\gamma / g$ have to follow the principle of least action and keeps in a narrow $\delta \mathrm{K}$ as we discussed before. And due to this narrow $\delta \mathrm{K}$, we then have to make the following modifications. As the conventional theory says, the more increase of $\mathrm{g}$, the phase separation becomes easier, and diverse molecular agglomerations will then tend to a maximum stretching state instead of a folding state. Now due to the relatively narrow shifting of $\mathrm{K}, \mathrm{K}=\gamma / \mathrm{g}$ tends to a constant. We can get three conclusions: (1) the ratio of surface tension $\gamma$ and gravity $g$ tend to the constant $\mathrm{K}$; this is the Principle of Least action we mentioned before. (2) the more surface tension $\gamma$ means, the more complex of the structure, and more of the gravitational binding $\mathrm{g}$, so $\gamma$ directly represents the complexity of a structure. (3) different molecular structures need different gravitational binding to issue their oscillation functions, $10^{9}$ level of gravitational binding in bio-systems come from here. Therefore, in bio-systems the modified conclusion is; the more $\mathrm{g}$ or the more surface tension induced gravitational binding, the more folding at this structural level (diverse free cell oscillation migration state still can be regarded as a special folding), and from this structural folding shifts to another structural folding, also need gravitation binding as driving forces; this conclusion is a little bit different to the non-living system. In old age, while constant $\mathrm{K}$ retrograded, this conclusion is gradually retrograded to the non-living system conclusion. Compare the equation $\mathrm{R}_{\text {mobility }}=\rho \mathrm{gV}=\rho \mathrm{g} R_{h v}$ and the equation $\gamma=\mathrm{g} \bullet \mathrm{K}$, we can use $R_{\text {mobility }}$ or $R_{h v}$ for estimating surface tension or gravitational binding if laboratory-scale HiGee is available (Suppl. ExpL. 4). The ratio of two genes' relative mobility in vitro to the same stationary phase will be similar to their relative mobility in vivo; albeit we can't make in vitro stationary phase equal to in vivo environment, such an in vitro ratio can be used for deciphering two genes' affinity functions. (Ancient physical strike technology also can be modulated by

\section{Volume 8 Issue 11, November 2019}




\section{International Journal of Science and Research (IJSR) \\ ISSN: 2319-7064}

ResearchGate Impact Factor (2018): 0.28 | SJIF (2018): 7.426

relative mobility; physical strike hits on certain part of the body, if the relative mobility shifting of all the suffering tissues induced by strike falls within certain range, the surface tensions and gravitational binding of all these tissues will get strengthened; if such shifting out of range, bruises or hurt will be induced, then the suffering tissues need the gravitational binding from other places to recover. Physical strike training start from young can tighten $\mathrm{k}$ and strength both surface tension region and gravitational binding; also, the training can maintain to very old age. However, if not start before 35 years old, it will gradually lose the benefit to health unless some compensation methods can recover CSF-ligament conjugation strength again. The body resistant of physical strike still by means of inversion, all the suffering surfaces can be modulated by bio quantum paths with inward surface tension; while strike reaches these inward surface tensions inversed to outward direction to resist physical strike and transfer them to horizontal distributors, the second layer still equally inversion in the same way, from the surface till the strike attain depth, each layer of bio quantum paths just use such an inversion way for resistant, while the strike leaves, they recover back to the original inward direction, this is a relay inversion.)

In bio-systems, palindromes arc could be regarded as another phase of genes since the percentage of which is increasing with evolution. Over $98 \%$ of the human genome is noncoding [17], while only $20 \%$ of a typical prokaryote genome is noncoding. Palindromes are "conservative sequences", and coded genes are mainly "non-conservative sequences" based on their fitness with bio quantum paths. While issuing function, it must superimpose non-conservative oscillations into conservative oscillations by splicing. This also means that coded genes are evolving from palindromes, coded genes issue higher oscillation levels of functions, and palindromes supply recovering capability for these levels. Without palindromes, coded gene functions will be quite unstable and ephemeral. Gene regulations are also following such a pattern. Restriction enzymes or restriction endonucleases are examples. These enzymes are found in bacteria and archaea to provide a defensive mechanism against invading viruses. Type II restriction enzymes (homodimers) are functioning on palindromic sequences [18]. This means cutting on such sequences will be much more effective than on other sites. Conventional evolution theory describes LUCA as a common ancestry and never regards the virus as a life. However, much evidence demonstrated that palindromic sequences are essential to the viral life cycle [19-23]. In the gut, they even make use of palindromic sequences from the secondary host: human beings, along with their utilizing of the palindromes in primary hosts: gut microbes [24]. Without palindromes, they possibly even can't invade hosts. The driving bio quantum paths are therefore evidently come from more than one organism. These prove that palindromes are physically modified. Life evolves with palindrome percentages is to increase the genomic "surface tension region" rate. It is imperative to know the relative mobility of each gene related to palindromes and to other genes while they interactions. That is for deciphering the surface tension and gravitational binding of genes, even more important than the linear nucleotide sequence.
$\mathrm{CNV}$ is a phenomenon in which sections of the genome are repeated, and the number of repeats in the genome varies between individuals in the human population [25]. It also happens in board species include E.coli [26]. Approximately two-thirds of the entire human genome is composed of repeats [27], and $4.8-9.5 \%$ of the human genome can be classified as CNV [28]. It is a type of structural variation with duplication or deletion event that affects a considerable number of base pairs and is generally categorized into short repeats and long repeats. Short repeats include mainly bi-nucleotide repeats (two repeating nucleotides e.g. A-C-A-C-A-C...) and tri-nucleotide repeats. Long repeats include repeats of entire genes. Genome-wide studies have concluded that there is a minimal spatial bias of the genomic location of CNV [29]. One example of short $\mathrm{CNV}$ is the tri-nucleotide repeat of the $\mathrm{CAG}$ base pairs in the Huntingtin gene that is responsible for the neurological disorder Huntington's disease[30]. Once the CAG tri-nucleotide repeats more than 36 times, Huntington's disease will likely develop in the individual, and it will likely be inherited by his or her offspring [30]. The number of repeats of the CAG tri-nucleotide is correlated with the age of onset of Huntington's disease. One example of a whole gene repeat is the alpha-amylase 1 gene (AMY1) that encodes alpha-amylase which has a significant copy number variation between different populations with different diets [31]. It is also one of the few genes that had been displayed convincing evidence which correlates protein function to its copy number [31]. It was found that the high starch diet populations had a significantly higher ( 2 times higher) average AMY1 copy number than the low starch diet populations ${ }^{31}$. The recent origin of the multi-copy AMY1 gene implies that depending on the environment, the AMY1 gene copy number can increase and decrease very rapidly relative to genes that do not interact as directly with the environment [32]. However, for the causes, mechanisms, and correlation with certain diseases, etc., there are a substantial amount of reports but no consensus theory. Back to Eq. $4 \mathrm{CNV}$ will be easily explained. We can write Eq. 4 into:

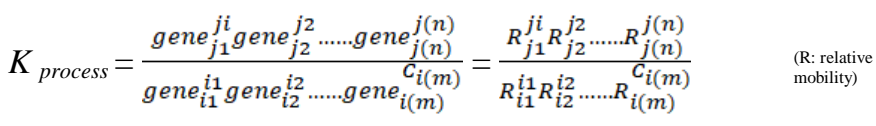

Clusters of functionally related genes, from gene ${ }_{\text {in }}$ change to gene $_{j m}$ for certain process can be given a $K_{\text {process }}$, each copy number was on the power of each gene. (If introns or exons or even short repeats join the process, we just write involved genes into intron or exon or even bi-nucleotide, tri-nucleotide repeats format then CNV roles keep the same.) The shifting of CNV is due to Le Châtelier's effect to keep constant K. What drives the CNV shifting are physical surface tension regions which could possibly be measured with $R_{\text {mobility }}$ or $R_{h v}$. Following Eq. 4 even "DNA damage" is a quite ambiguous concept; DNA follows the same surface tension rules with other things. We should also note that among the neurons in the human brain, somatically derived $\mathrm{CNV}$ are frequent ${ }^{33}$. CNV show wide variability ( 9 to $100 \%$ of brain neurons in different studies). Most alterations are between 2 and $10 \mathrm{Mb}$ in size, with deletions far outnumbering amplifications [33]. CNV appears to be higher in brain cells than in other cell types [33]. This is not epigenetic shift but real sequence shifting [33]. 


\section{International Journal of Science and Research (IJSR) \\ ISSN: 2319-7064 \\ ResearchGate Impact Factor (2018): 0.28 | SJIF (2018): 7.426}

Brain mosaicism strongly proves the bio quantum path model and the splicing process.

Upgrade Newtonian motion \& rest into bio-system superposition stationary structure

The term "metastasis" has been recorded for near three centuries (1727-1850, "metastasis of milk" presented in publications, albeit at that time, it was not concerned with cancer.). Till today people normally know that metastasis is migration and proliferation of cancerous cells (epithelial origin) to different in vivo sites. However, whether metastasis is only specified in cancerous cells? If so, which kind of factors can offer cancerous cells that "super mobility and implantation capability" which normal stem cells lack? Such factors have never been revealed; we then have to believe that cancerous cells and normal stem cells are in the same capability levels for mobility and implantation. Top of (Fig.1f) is from a study for the distinctive role of Wnt $5 \mathrm{a} / \mathrm{Wnt} 5 \mathrm{~b}$ in mouse embryonic development [34]. The Wnt signaling pathways are a group of signal transduction pathways from cell surface receptors till genomic regulation level; this means they will vertically pass all horizontal surface tension regions at the cell levels. We can see the blue staining is never restricted to a certain locality but also not attain full region, establish some regulation spectrum. Wnt signaling pathways at the cell levels impact on to the somatic level or the spinal cord. At the bottom left is a breast cancer metastasis to the lung region. We could see both normal cells and cancerous cells have far reach mobility; establish a proliferation spectrum. (If we choose other stem cell indicators, we still can get a similar spectrum. Even physical strike on one site will be distributed in such a board spectrum on diverse sites if the strength is within the tolerant range.) Evidence also from other sources, metastasis is reported as the result of inflammatory processes by abnormal immune cells [35]. The existence of metastatic cancers without primary tumors also suggests that metastasis is not specified in malignant cells [36]. (This confirms what we mentioned before; cancer is originated from constant $\mathrm{K}$ asymmetric retrograde, or arise from normal cells.) To understand cancerous and normal cells in mobility, we need to modulate motion and rest concepts in bio-systems.

Early in the Newtonian system, motion is defined as the change in position of an object with respect to its reference frame in a given interval of time. If the position of a body is not changing with respect to a given frame of reference, the body is said to be at rest state. A choice of the reference frame is free for "inertial frame of reference". The concept of energy conservative naturally comes from here. However, the Newtonian system is not for surface tension region; the rigid body concept of it even intentionally gets rid of surface tension. Einstein modified the Newtonian system, still didn't refer to surface tension, albeit tensor he usually used is a standard tool for that; just his gravitational waves can be indirectly regarded as the binding surface tension region (we'll refer to later). The quiddity of quantum mechanics is actually a discipline for surface tension; just no one realizes that. (Double-slit can be performed by water or oil surface is stronger evidence. Both Planck's blackbody radiation and Einstein's photoelectric effect are actually concerning "surface tension". Quantum mechanics has already very close to transcending thermodynamics; just due to such "non-realizing", it restricted itself in the microcosmic world and even left some pending issues. Right bottom of (Fig. 1f) is a metal bar; following the Second Law of Thermodynamics, it is impossible to get one end of it in higher temperature and another end in a lower temperature at equilibrium. However, an open system insides surface tension region can get such "fluctuation equilibrium". Quantum mechanics failed to modulate that.) Therefore, this paper largely comes from modified quantum mechanics. Also, quantum mechanics takes unitary time evolution, difficult to modulate "inversion"; we have to integrate some music, the only inversion discipline in the world. Back to (Fig.1d), bio quantum paths depend on a general strengthening \& weakening effect for splicing out resistance. It at least composes of two parts, a motion part and a rest part. Now the motion concept is no longer diverse types of Newtonian motions; the only motion is a strengthening \& weakening or "fluctuation" motion. The rest part is the inseparable sustaining signal part while "fluctuation" is running. While a lot of bio quantum paths composited into a bio-system, a holistic "stationary" structure that sustains all levels of fluctuations still exists, and the stationary concept is no longer a reference we can choose freely; it splices out the resistance for the whole system. A typical type is a spinal cord we see; it origins from the cell bi-membrane model.

(Fig.1g(1)) is a string oscillation figure possibly even present earlier than the history of human written languages (Suppl.ExpL.5). (Fig.1g(2) shows series bio quantum paths with corresponding "splicing number" equal to the "wave number" of the oscillation strings (only draw the $n=3$ and $n=6$ bio quantum paths). Splicing number is the number of periods in $\mathrm{Z}$ dimension while $\mathrm{x}, \mathrm{y}$ dimension finish one cycle. Now that stationary structure can't be separated with conservative energy, we can go back to earlier quantum mechanics. This discipline had experienced four stages: Planck's law, Einstein's photoelectric effect, Bohr model of the atom, and Schrödinger's model. The major success of the Bohr model was in explaining the hydrogen spectrum. It believed that electron jumping among "orbits" could emit or absorption energy. Such "orbits" could be regarded as a series of strings in (Fig.1g(1)) or bio quantum paths in (Fig.1g(2)), for non-living beings energy possibly can be jumped in this way since it's just energy that not concerns with structures; however, for living beings we can see their splicing number is different, in normal conditions energy need first transfer with same "splicing number" or frequency, that is horizontal transfer to the attaching structures, and the feedback energy goes to corresponding splicing number. Vertical "jumping" similar to Bohr's model means drastic structure and energy shifting in the body. Energy is no longer something that can transfer freely, the transfer of energy in bio-systems profoundly concerns with structure and oscillation frequency by Le Châtelier's effect. Schrödinger himself disliked energy vertical "jumping"; therefore, wave function replaced "orbits" and allowed the electrons to occupy three-dimensional space. Three quantum numbers (coordinates) were needed to describe the distribution of electrons. (Principal quantum number (n), azimuthal quantum number $(\ell)$, and magnetic quantum number $(\mathrm{m})$, later spin quantum number $(\mathrm{s})$ is also added into.) However, the problem of the fragileness of the quantum states to enter in a macrocosmic world still remains.

\section{Volume 8 Issue 11, November 2019}




\section{International Journal of Science and Research (IJSR) \\ ISSN: 2319-7064}

ResearchGate Impact Factor (2018): 0.28 | SJIF (2018): 7.426

From $E \Psi=\widehat{H} \Psi$, energy becomes $\left|E_{n}\right\rangle$, and certain $E_{k}$ is presented by probability which measurement will induce to collapse into certainty.

Possibly due to still not satisfied with Copenhagen's interpretation, Schrödinger released his legendary "cat". After that release, quantum mechanics failed to convincingly deny the legend that before opening the box, the cat is in a "dead-and-alive superposition state". Quantum mechanics itself has then to become a "dead-and-alive superposed discipline" till today. Now we can slightly modify this thought experiment to find some hidden premises. We put two cats, one male and one female both with good fertilities into a box with two chambers separated by a gate, the device which controls the gate is still Schrödinger's radioactive device, just in one hour decaying time there is $50 \%$ chance of opening the door. Then we'll see while opening the box how many cats will get. One possibility is just a male and a female cat; one possibility is beyond the couple we get a few extra baby cats. However, this case is a little bit different from that of conventional Schrödinger's cat. Generally, a cat from mate till delivery babies takes around 70 days, the male and female cat still need time to "fall in love" and get pregnant. We just suppose 70 days for the whole process. So after one hour of the decaying of a radioactive element, then within 70 days no matter how hard and how many times we open the box and whether the Schrödinger device is triggered or not, the result will definitely no more than two cats. Therefore there is no such story as "superposition of states collapse to a concrete state". After 70 days could possibly get extra baby cats; however, the chance still quite lower than 50\% (which previously set to radioactive decaying) since diverse causes will significantly impact the probabilities. Both cases are opening the box for observation, why one is legendary probability story, and another is certainty in a long period of time? We could easily understand the only difference is the time. If the legendary claim that "observation can affect a quantum wave function", then it definitely can only impact the events which happen in a time frame quite shorter than that of the observation time. Suppose open the Schrödinger's box for observation needs 3 seconds; this observation can only possibly affects events which happening time is far less than that of $3 \mathrm{~s}$. As in the baby cats' case, it takes 70 days for delivery, and if we still want arbitrarily "probability condition", the only way is to increase the observation time to $\gg 70$ days. In the Bohr-Einstein debates, there is a topic about whether Moon is presented if we don't observe it. Actually, at that topic, Einstein is correct; no matter we observe or not, Moon exists there. Since a glimpse observation time is only second and the Moon's existence is quite long. Suppose one glimpse is $1 \mathrm{~s}$, then this glimpse only possibly affects events which happen in millisecond or microsecond; it is definitely impossible to affect the Moon that had existed there for 4.4-4.6 billion years. If someone really wants to make the existence of the Moon becomes a probability event in his eyes or even want "probability can be collapsed by his glimpses", he possibly needs to become a "superman" that a glimpse takes at least 100 billion years, then his lifespan will attain $3 \times 10^{9}$ billion years. However, right now, the known age of our universe since the Big Bang is only 13.8 billion years. From here, we could easily understand that the superposition of the quantum state is conditional with time, and the collapse of the quantum state must also observe the above time frame. Back to the case, we could regard a pregnant female cat as a quantum state superposition and delivering babies as "collapse to non-superposition state" by the number of cats as a physical parameter, then not in the right time no matter how hard and how many times we open the box for observation, can never make the "collapse" (delivery babies) happen earlier. This means that quantum superposition collapse absolutely follows the above time frame. The prerequisite condition for any observation or event can induce wave function collapse is their happening time must quite longer than that of collapse time. And this is only a necessary condition instead of an NSC. (From here we can understand, quantum mechanics will never take superposition state as a stationary structure since its wave function is too fragile.) Another problem for quantum mechanics to be applied in bio-systems is the unitary time evolution. (It is a restriction to ensure the sum of probabilities of all possible outcomes of any event equals to one, $\int_{-\infty}^{+\infty} \Psi^{*}(x) \Psi(x) d x=\int_{-\infty}^{+\infty}|\Psi(x)|^{2} d x=1$.) This means the stability of an event is decided by the unitary of event probability. However, bio-systems take a totally contrary way for stability, due to constant $\mathrm{K}$ binding some biomaterials' stability depends on other biomaterials' speed of disappearing, or anti-unitary processing. Like foods become excrements leave the body, the speed of excrements leaves the body after removing part of the surface tension regions or gravitational binding from the mixtures strengthens the system stability.

Besides the unitary problem, the major jinx is gravity. Some people like to claim that quantum mechanics has solved all types of physical interactions except gravity. However, double-slit can be performed with water or oil surface, this means surface tension drives the experiment, and surface tension can't separate with gravity; oddly, wave function fails to refer to gravity for so many years. In (Suppl.Exp.7), cutting the spinal column of an animal can quickly release the gravitational binding; it proves that quantum superposition itself is a process of binding gravity. Albert Einstein has questioned the completeness of quantum mechanics by the EPR paradox [37]. Till today most of the people believed that Einstein was wrong since various experiments of quantum entanglement gave them specious support. However, if we carefully check all the experiments we can easily find, these experiments just partially proved that quantum entanglement did present at a certain distance. None of them have shown that the "spooky action" can be presented without the help of gravity. Following our model, quantum entanglement is just a natural result of Earth's gravity (a large surface tension region). Based on all nowadays available experiments, it is really too early to say that Einstein is wrong, albeit his paper about the EPR paradox was not fully accurate. Unless some experiments can prove that "quantum entanglement" can stably penetrate through the gravitational field of another planet which not initiating the quantum state, such as entangle to device put on Moon or Mars, this process can then be qualified with the word "spooky" which granted by Einstein. If finally, quantum entanglement can't penetrate through the gravitational field of another planet which not initiates the quantum state, then it means Einstein's intention for seeking "hidden variable" becomes a success, the hidden variable of quantum state 


\section{International Journal of Science and Research (IJSR) \\ ISSN: 2319-7064}

ResearchGate Impact Factor (2018): 0.28 | SJIF (2018): 7.426

superposition falls on "gravity" and its derivatives. In the field of quantum mechanics, Einstein's intuition had transcended his predecessor Planck in the discipline; just his successor didn't reach his intuition level. In Bohr-Einstein debates, he still didn't find the solution for defending; just from his intuition, he believed that something was incomplete. Today if we back to history, we have to admit that almost everything from his intuition in the debates is correct. For Bohr, we just put a tiger behind him; he would possibly have to change his mind. The fact that he didn't observe or even think of the tiger totally can't prevent the animal from killing him. Face to face, a tiger will possibly hesitate in capturing under some conditions; once face to the back of a person, a tiger will greatly increase its motivation for hunting. Now it's no longer the observation the controls the attack; it is the more he unobserved the tiger, the more he will get the attack.

The most important for all, "whether a quantum state can present independently in a non-isolated environment"? Actually, there are seven correlated questions inside this question that can't be separated; we discuss them together:

1) Definition of motion and rest in a bio-system

2) Where the time-independent Schrödinger equation comes from

3) "integer" or "integer variable function" quantization of a quantum system

4) The recovering capacity of a quantum state

5) The stability of the superposed quantum states (unitary or "turnover rate" superposition)

6) Time evolution or time commutation

7) "Regenerative" wave-particle duality and the range of quantum state (macro- or microcosmic)

For (1), we have discussed before that in bio-systems, the motion (fluctuation) and rest (stationary) are inseparable for sustaining the function. For (2) following such modified concepts of "fluctuation" and "stationary", if we wish to apply the quantum mechanics in bio-systems, the quantum state can't be present independently; Schrödinger equation should have certain accompanying structures. It is those attaching structures that make the stationary state present in certain environmental conditions. The fragileness of quantum state, such as wave function collapse, comes from the shortage of attaching structures. Now we discuss (3), earlier quantum mechanics in Planck's and Einstein's studies are from the macrocosmic world. After Bohr and Schrödinger, it began to deal with the microcosmic world. Copenhagen's interpretation eventually makes people totally puzzling about how to apply such "upgraded" mathematics back to a macrocosmic world. Planck's Law published in 1900 can be written as:

$$
I_{v}(v, T)=\frac{2 h v^{3}}{c^{2}} \frac{1}{e^{\frac{h v}{k T}}-1}
$$

$\left(I_{v}, v, T, h, c, k\right.$, are radiance, frequency, Planck's constant, speed of light, Boltzmann constant, resp.)

This formula, in the limit of low frequencies, tends to the Rayleigh-Jeans law and in the limit of high frequencies, tends to the Wien approximation; means it is based on enough empirical evidence. The prerequisite condition of this formula becomes the foundation of quantum mechanics, $E=n h v$, $(5$ years later Einstein successfully uses it to explain the photoelectric effect, the energy of the emitted electrons depends on the frequency of the photons instead of the intensity of the incoming light.). A blackbody is indeed a surface tension model, energy trapped by it becomes radiation; the trapping forces could be regarded as inward surface tension. Planck's model is derived from the classical simple harmonic oscillator model, $E \Psi=\widehat{H} \Psi . E=n h v$, is an "integer function". For bio-systems, we then need another formula $f_{n}=$ $f_{1} \times \sqrt[12]{2^{\mathrm{n}-1}}$ (Suppl. Expl. 5) for frequency energy since all based on simple harmonic motion. Also, $f_{n}=f_{1} \times \sqrt[12]{2^{\mathrm{n}-1}}$ can be adapted into Planck's law and becomes, $E=\sqrt[12]{2^{\mathrm{n}-1}} h v$, $n=1,2, \cdots \cdot n$; for integer, it recovers back to original equation $E$ $=n h v$, just some non-linearity values presented. Einstein's photoelectric effect reveals that frequency decides the pathway of energy transferring. It can also be explained by $E=$ $\sqrt[12]{2^{\mathrm{n}-1}} h v$ replaces $E=n h v$ for the whole process. (Work function $W=h f_{0}=\sqrt[12]{2^{\mathrm{n}-1}} h$ where $f_{0}$ is the threshold frequency of the metal. The kinetic energy of an ejected electron is then $k=h\left(f_{k}-f_{0}\right)=h\left(\sqrt[12]{2^{\mathrm{k}-1}}-\sqrt[12]{2^{0-1}}\right) f_{\text {ground }}$. Einstein's photoelectric effect theory also concerned with surface tension region; electrons bound to metal are activated by certain frequencies from incident photons and then jump out of the surface of the metal, which can be modulated by bio quantum path. From here, we can understand, quantum mechanics is actually a surface tension discipline. Not realizing the disciplinary surface tension quiddity, quantum mechanics had to confine itself in the microcosmic world. And even in that world, it is not always successful, yet left some unsolved issues.

Music harmonics directly based on simple harmonic motion, the above "integer to integer variable function" modification is not only very convenient for harmonic analysis (Suppl. ExpL. 6) but also agrees with our definition of motion and "stationary" in bio-systems; no matter at which levels, while $\mathrm{n}=12 \mathrm{k}+1(\mathrm{k}=0,1, \cdots \cdot \mathrm{K})$ or the integer is present in the formula, it is stationary structure, and while " $n$ " equals to other integers, they become the fluctuation structure which resides on the integer "stationary" state. For bio-systems $\left|N_{12} \sqrt{2^{\mathrm{n}-1}}\right\rangle$ as a quantum state, the non-integer part is the origin of the recovering capacity or Le Châtelier's effect we mentioned before; therefore, more robust. A lifespan of 100 years means the highest level of the quantum superposition (spinal cord) can maintain 100 years on proper environmental conditions. For the non-living $|n\rangle$ quantum state, the fragileness comes from lacking the non-integer part. This is what (4) refers to. Now we can discuss (5), the stability of the superposed quantum states. The superposition principle states that any two (or more) quantum states can be superposed together and the result remains a quantum state. This is only a mathematical axiom. If quantum mechanics is a surface tension discipline and what superposed are certain surface tension regions like in bio-systems, this principle becomes challenging. (The critical problem is, if we require every superposition must present constructive \& destructive interference pattern or the strengthening \& weakening effect as prerequisite condition, then in a non-isolated system with the increase of quantum number " $n$ ", substantial numbers of quantum state superposition will become "ineffective superposition" which constructive $\&$ destructive interference patterns are gradually

\section{Volume 8 Issue 11, November 2019}




\section{International Journal of Science and Research (IJSR) \\ ISSN: 2319-7064 \\ ResearchGate Impact Factor (2018): 0.28 | SJIF (2018): 7.426}

decaying. Senility is the increasing of "ineffective superposition"; that principle just means that life can be eternal.) This is why this discipline has never clarified the conditions for the time-dependent or time-independent state. We could also understand from an identical particle model from quantum mechanics, the superposition of two particles is:

$$
\left|n_{1}, n_{2} ; S\right\rangle=\text { constant } \times\left(\left|n_{1}\right\rangle\left|n_{2}\right\rangle+\left|n_{2}\right\rangle\left|n_{1}\right\rangle\right)=1 /(\sqrt{2}) \times\left(\left|n_{1}\right\rangle\left|n_{2}\right\rangle+\left|n_{2}\right\rangle\left|n_{1}\right\rangle\right)
$$$$
\left|n_{1}, n_{2}, A\right\rangle=\text { constant } \times\left(\left|n_{1}\right\rangle\left|n_{2}\right\rangle-\left|n_{2}\right\rangle\left|n_{1}\right\rangle\right)=1 /(\sqrt{2}) \times\left(\left|n_{1}\right\rangle\left|n_{2}\right\rangle-\left|n_{2}\right\rangle\left|n_{1}\right\rangle\right)
$$

Bosons, occupy symmetric states and fermions occupy antisymmetric states, for "n" particles:

$$
\begin{aligned}
& \left|n_{1}, n_{2} \cdots \cdots n_{N} ; S\right\rangle=\sqrt{\frac{\Pi_{j} N_{j} !}{N !}} \sum_{p}\left|n_{p(1)}\right\rangle\left|n_{p(2)}\right\rangle \cdots \cdots\left(\mathrm{n}_{p(N)}\right\rangle \\
& \text { Or } \left.\quad\left|n_{1}, n_{2} \cdots \cdots \cdot n_{N} ; A\right\rangle=\frac{1}{\sqrt{N !}} \sum_{p} \operatorname{sign}(p) \mid n_{p(1)}\right)\left|n_{p(2)}\right\rangle \cdots \cdots\left(\mathrm{n}_{p(N)}\right\rangle
\end{aligned}
$$

All these particles are "indistinguishable" and establish a quantum state follow Eq. 18, could be symmetric, antisymmetric or even exchange symmetric. However, in bio-systems, all these particles (all biomaterials such as DNA, RNA or cells, etc. can be physically expressed into bio quantum paths, here we just regard one bio quantum path as one identical particle.) will be automatically grouped by the strength of "Le Châtelier's effect" (As the double-slit experiment in the right bottom of (Fig.1f), the interference patterns on the screen have already failed to distribute evenly. Such uneven of superposition pattern will definitely decay the interference effect with the increasing of " $n$ " number.). As a result, particles with higher "Le Châtelier's effect" will be pushed in one group, the other particles are then offered "turnover rate" for them. (Definitely, with the evolution of time, these particles with higher "Le Châtelier's effect" could possibly become "turnover rate" particles for other particles. Therefore the system only leaves "turnover" structures and no longer the particles which establishing the quantum state.) This is the "anti-unitary" or "turnover" superposition in bio-systems. A cell is such an "anti-unitary" or "turnover" superposition structure that migration based on environmental signals and own stationary eigen-surface tension region (Suppl. ExpL. 7).

For (6), time evolution in quantum mechanics is unitary and in living systems is anti-unitary. The anti-unitary or commutation time offers bio-systems board inversion processes that stable the surface tension structures. The more inversion of a process or a structure, the more stability it will get. The retrograde of a structure or a network is induced by the retrograde of inversion. Mathematically, quantum mechanics have written down the commutation of diverse physical parameters; however, it never believes the number of commutation rounds of the parameters of a process is the stability of this process, so it takes unitary time. This is contrary to evolution.

For (7) we should understand that wave-particle duality is a "regenerative" property, slightly different from that of the routine explanations. Given a bundle of particles A and a series of wave B, even in the macrocosmic world, we can't simply say A presents particle characteristics and B presents wave properties. The A or B we see just based on our previous experience, not the performance of group characteristics of either A or B's on a system. Without an interacting system, both $\mathrm{A}$ and $\mathrm{B}$ are neither particles nor waves. Back to the right bottom of (Fig. 1f), there is a Gaussian curve; all the universal events will fall into it with enough repetitive numbers. This is a quite general fluctuation; if such a fluctuation from certain events can be resonated with a large system, it will demonstrate wave characteristics. And under an integrated wave environment of certain events, the superposition of waves will demonstrate particle properties (the number of wave peaks becomes the number of particles) in certain conditions. This is the wave-particle duality in bio-systems, never concerned with micro- or macro-scales, only concerned with the existing of the resonating system and surface tension region inversion. (The quiddity of such wave-particle duality is actually the conditions which constructive \& destructive interference or a more general strengthening \& weakening effective could happen, definitely not concerning with the raw materials we observed are particles or waves; both previous observed "particles" and "waves" can issue constructive \& destructive interference under certain conditions if a resonating system is presented.) In quantum mechanics, the principal quantum number is the number of electronic shells in an atom to describe the electrons' state. In bio-systems, it becomes the number of vertebrae. We also need to understand that vertebral quantum numbers must be a "regenerative" number and not the number of vertebrae we see on the spinal cord. (For an alive or dead animal, the vertebral number is the same, but the "regenerative" quantum number is totally different.) The spinal cord in nature has been evolved for billions of years from the first evidence of life [2] untill the first vertebrate Haikouichthys presented in 525 million years ago [38]. The applications correlate with the spinal cord in human society have also experienced thousands of years for progress. (From the unfledged I Ching, the origin of Chu's constant till the finalization of the Wudang physical training system 590 years ago, ancient Chinese culture had advanced in practices. Theoretical progressing from western culture started from earlier Newtonian mechanics, Einstein's theory, and quantum mechanics till biosciences. This ungraded Newtonian motion \& rest definition comes from the superposition of multi-culture \& multi-discipline for thousands of years, can't be worked out by a single discipline or even a single culture independently. We'll gradually supply historical and modern evidence later.)

Now back to the metastasis we discuss at the beginning of this section, we could easily understand that both normal stem cells and cancerous metastasis cells have stronger mobility and implantation capability, just normal stem cells based on spinal cord for such "capability" and cancerous metastasis based on a "deformed spinal cord" for that. Some people suggested using stem cells to medically repair spinal cord injuries. This is a routine manner in conventional medical research, just unfeasible. There are substantial technological problems in stem cell applications (Suppl.ExpL.8); even if we can overcome all these technological challenging, major challenging still remains. The spinal cord binding a lot of attaching structures in the body; its injury means all the attaching structures have also impacted. Even if we can overcome all the problems of stem cells and "repair" the spinal cord biologically, we still can't "repair" all the injuries inside attaching structures. (As (Fig.l) of (Suppl. ExpL. 4), the spinal cord composed of all the surface tension converging region of the body, its damage means the surface tension regions of the whole body get hurt. To recover all the surface tension regions is quite challenging for nowadays technology.)

\section{Volume 8 Issue 11, November 2019}




\section{International Journal of Science and Research (IJSR) \\ ISSN: 2319-7064 \\ ResearchGate Impact Factor (2018): 0.28 | SJIF (2018): 7.426}

While Planck wrote down his black body radiation formula and also in later quantum mechanics, people just believe that "Planck's constant $h$ is the smallest scale of energy transferring", seems never refer to the "fluctuations" inside it albeit simple harmonic motion model is extensively utilized. Planck's constant can be empirically measured by black body radiation or Einstein's photoelectric model. Now, based on the new interpretation of wave-particle duality in bio-systems, we could understand that " $h$ " is the "least fluctuation which can regenerate with the whole system". Following such a definition, we can find Planck's constant in bio-systems; the minimum discernable fluctuation is likely to be a palindrome or an intron in scale. CRISPR repeats typically range from 28 to $37 \mathrm{bps}$ [39], and the shortest intron is 30bps [40]. Thus the least "fluctuation" in a bio-system could be the horizontal projection of $30 \mathrm{bps}$. $30 \mathrm{bp}$ in a straight line is around $30 \times 3.4$ $\AA \approx 100 \AA, 10^{-8} \mathrm{~m}$, suppose this $30 \mathrm{bp}$ roughly composed of the two sides of an equilateral triangle then the $h_{b i o}$ should be $15 \mathrm{bp}$, around $50 \AA=5 \times 10^{-9} \mathrm{~m}$, with oscillation range. This scale equals to $7.5 \times 10^{24}$ Planck quantum or 50 water molecules in a line. We can then write "conservative energy" or "frequency energy" in as:

$$
\begin{gathered}
E_{\text {conservative }}^{\text {bio }}=\sqrt[12]{2^{\mathrm{n}-1}} h_{\text {bio }} \omega \\
\left(\mathrm{n}=1,2,3, \text { to } \mathrm{n}, h_{\text {bio }}=h_{\text {planck }} \times 7.5 \times 10^{24}=5 \times 10^{-9}\right)
\end{gathered}
$$

Eq. 19 means in bio-systems least fluctuation scale which can resonate with the whole system becomes a modified Planck constant $h_{\text {bio }}$. (Such constant $h_{\text {bio }}$ is possibly only fit for all eukaryotes and most prokaryotes with enough percentage of palindromes. For the most primitive types of life, the $\mathrm{h}_{\text {bio }}$ is possibly only three nucleotides; however, three-nucleotide is not in a straight line. We just take $1 / 10$ of $5 \times 10^{-9}$ from the previous estimation of $30 \mathrm{bp}$, which is $5 \times 10^{-10} \mathrm{~m}$.

Mitochondrial and chloroplast sequences should take this scale due to their prokaryotic properties, so we can write $h_{m t}=$ $h_{\text {chloro }}=5 \times 10^{-10}$. This conclusion is also supported by the fact that triplet code as a three-nucleotide codon binds a single amino acid with some exceptions which allow insertion [41] and short CNV repeats we mentioned before are bi-nucleotide or tri-nucleotide repeats [28]. Genes superposed on palindromes for recovering capacity, and triplet code can take insertion for its recovering capacity. We should note that both $h_{b i o}$ and $h_{m t}$ are only for the ideal or young stage. Senility is a process of decaying of the percentage of in vivo compromised oscillation "wavelengths" which can fall into $h_{b i o}$ or $h_{m t}$ in a bio-system.) The physical significance of $h_{m t}$ just proves again that energy in bio-systems is frequency controlled "fluctuation" energy. $h_{m t}$ equal to around 5 water molecules in a line, that means no matter which kind of energy, either from water molecule oscillation or from any chemical bonds or even compound reactions in water, they must possess a least "fluctuation" scale for the energy exchange. In the ideal conditions, if the "fluctuation" scale is lower than 5 water molecules in size, then no energy can be transferred under all conditions. Planck or Einstein's systems can exchange energy with " $h_{\text {Planck" }}$ "scale due to there are much less constant $\mathrm{K}$ binding and lower recovery requirements for their system. Bio-systems with the above two requirements have to utilize frequency energy with at least $7.5 \times 10^{23} \mathrm{~h}_{\text {Planck }}$ scales. (This number is a little bit close to the Avogadro constant $6.02 \times$ $10^{23}$, possibly in the future, it needs to consider this constant and enough empirical data for modifications. That implies life depends on a scale which equivalent to 1 mol Planck's constant in a queue as the least frequency energy fluctuation scale. We'll also note that this is just a theoretical ideal condition; in a real condition due to age or various factors, the minimum fluctuation scale should be shifting in a range which enlarged by senility.)

\section{Upgrade Einstein's frequency energy into network binding copy number inversion, horizontal \& vertical inversion of surface tension region or gravitational binding}

Einstein's frequency energy comes from his photoelectric effect model, not the indiscriminate energy conservative model. We've taken Einstein's original explanation and only change $n$ to $\sqrt[12]{2^{n-1}}$, now we'll use bio quantum path for modulation since the photoelectric effect is yet a surface tension problem. For electrons on the surface tension region of metal, its $\mathrm{z}$ dimensional oscillation composes of two parts fluctuating with $\mathrm{x}$, y plane: $z^{+}$and $z^{-}$can be given as follow:

$$
\left\{\begin{array}{c}
x=A_{x} \cos \left(\omega t+\varphi_{x}\right) \\
y=A_{y} \cos \left(\omega t+\varphi_{y}\right) \\
z=S_{z} \cos \left(\omega_{z} t+\varphi_{z}\right),\left\{\left\{\left|\frac{S_{z}^{+}}{S_{z}^{-}}\right|=k_{\text {photon, }}, \begin{array}{l}
z^{+}=S_{z}^{+} \cos \left(\omega_{z} t+\varphi_{z}\right) \\
z^{-}=S_{z}^{-} \cos \left(\omega_{z} t+\varphi_{z}\right)
\end{array}\right.\right.
\end{array}\right.
$$

The surface tension force is $\left|S_{z}^{+}\right|-\left|S_{z}^{-}\right|$, electrons are confined in the region means $\left|S_{z}^{+}\right|>\left|S_{z}^{-}\right|$, inward direction, as in (Fig.j) of (Suppl.ExpL.4). The confined electrons can absorb energy from photons when irradiated; there are various possibilities for $\left|S_{z}^{+}\right| /\left|S_{z}^{-}\right|$ratio shifting; however, following the Principle of Least Action, the surface tension region escaping should follow the least energy pattern since all the surface region confined electrons have integrated into Le Châtelier's effect by $\mathrm{x}, \mathrm{y}$ oscillations. (The higher Le Châtelier's effect actually comes from the higher gravitational binding of surface tension region.) The least energy type should be the energy of an "inversion" process, as $k=\left|S_{z}^{+}\right| /\left|S_{z}^{-}\right|$, just $S_{z}^{+}$and $S_{z}^{-}$exchange with each other, then the confined electrons will escape. The formula $k=\left|S_{z}^{+}\right| /\left|S_{z}^{-}\right|$or the surface tension region property decides the threshold frequency; we call the threshold frequency as the frequency energy of that surface tension region; it is actually the inversion energy of the bio quantum paths which represent for that surface. This way can explain the photoelectric effect well than before, also can be validated by various surface tension based experimental designs; just need some understanding of the kinetic state of bound electron follows bio quantum path modulation in the surface tension region. (It doesn't mean an electron can be separated into two parts with different ratios; it just means the relationships among bundles of electrons in surface tension region follow this modulation by oscillations. Actually, even Planck's blackbody radiation law can be solved by the same method. We can just simply use bio quantum paths to modulate "quantum".) Albeit Einstein didn't realize the inversion, he was the first one that explained the photoelectric effect, and the energy type used in this explanation had already different with that in his mass-energy equivalence, means a great step over; so we still call the least

\section{Volume 8 Issue 11, November 2019




\section{International Journal of Science and Research (IJSR) \\ ISSN: 2319-7064}

ResearchGate Impact Factor (2018): 0.28 | SJIF (2018): 7.426

surface tension inversion energy as Einstein's frequency energy.

Not only the photoelectric effect but also all other things in surface tension regions can physically modulate in the same way. Bio-systems have much more surface tension regions and more complex structures; even if the surface tension regions are always multi-phase complex instead of just electrons, they still interact with each other by inversions. Cells bound to tissues will still jump out of the surface and migration if activated by certain kinds of stuff such as RNA, cytokines, etc. to a certain frequency. And when their frequencies decreased to a certain threshold for some reason, they will back to certain sites with certain frequencies. The only restriction factor for energy transferring is frequency; the least energy is equally by means of inversion at the young state, senility just decaying such an inversion capability. (There is some spectrum between the threshold and the implantation frequency in bio-systems due to different Le Châtelier's radii. For an electron escape and reside back to a metal surface; there should be such kind of spectrum between different locations of the metal surface.) The membrane on the surface of tissue or organs just increases the escaping threshold values. While a small molecule, a protein, or a cell, etc., migration through many surface tension regions, each one still has its threshold frequency, then these multi-surface tension regions will relay cascade of inversion processes as the least energy transferring pattern. This is the Principle of Least Action for bio-systems. For the inversion of the confined electrons in the photoelectric effect, it is not easy to directly observe since electrons are so small. (The Cryo-EM can't capture the state of electrons just before emission since liquid nitrogen will first change the surface tension region.) However, for cell surface inversion frequency from the tissue surface, and earlier embryonic development inversion, can be easily observed in an experiment (Suppl.Exp.14). First, damage to the egg membrane will greatly impact the hatching rate, which means surface tension of the egg membrane plays a critical role in development. Second, a day 3 chicken embryo will implant the ceiling egg membrane for about one day which driving by surface tension; this is a somatic level inversion process. Later it will fall back and leave the blood vessel system on the egg membrane; the embryo begins to have two sets of blood vessel systems, one inside body and another on the egg membrane. These two sets of blood vessels are in an inversion relationship. The human embryo has a similar process with a few inversions; finally, still establish two sets of blood vessel systems, the placental and the somatic circulations, here two sets of blood vessels are for the inversion relationship. In both cases, the surface tension inversion of two sets of blood system steers the whole development. In plants, there are no blood systems; however, from ovule development, we still can see inversions. Developmental inversion is quite general in bio-systems; indisputably follows our surface tension model. Third, the AIT method that utilizing the inversion implantation stage successfully injects xenograft stem cells into the embryos and establishes chimera animals. This verifies that growth and immunity are driving by surface tension inversion since only at the implantation stage, xenograft stem cells have the same inversion frequency with that of the host, then can be injected without considering the immune rejection. The implantation stage includes board oscillation frequency unifying that utilizing by the on-egg membrane AIT injection method. All the resonating or frequency unifying is by means of inversion on surface tension regions. This experiment strictly proves the surface tension inversion frequency energy model at the cell and the embryonic development level.

To correlate and modulate the genomic level surface tension, we need the concept of horizontal \& vertical oscillation transfer. From the previous formula for HiGee, $R_{\text {mobility }}=\rho \mathrm{gV}=$ $\rho \mathrm{g} R_{h v}$, under similar gravitational binding $\mathrm{g}$ or complexity of a structure, we can use $R_{h v}$ to roughly replace $R_{\text {mobility }}$, here $R_{h v}$ means the horizontal and vertical splicing. In bio-systems, the gravitational binding difference of different genes is relatively small than those from the proteins and cells, so we generally can use $R_{h v}$ to represent the surface tension at the genomic level. (Such approximation will gradually close to actual condition with the increase of gene numbers.) The in vivo identifications of a gene by the system is the basis of its function, and it is based on "oscillation or quantum spectrum" similar to our physical choice of a musical instrument). (Fig.1g(3)) shows a CRISP/Cas9 [39] (Clustered Regularly Interspaced Short Palindromic Repeats) system. The CRISPR array comprises an AT-rich leader sequence followed by short repeats that are separated by unique spacers. CRISPR repeats typically range in size from 28 to $37 \mathrm{bp}$, though there can be as few as 23 bp and as many as 55 bp [39]. The size of spacers in different CRISPR arrays is typically 32 to $38 \mathrm{bp}$ (range 21 to 72 bp) [39], a little bit wider than that of the palindromic regions. There are usually fewer than 50 units of the repeat-spacer sequence in a CRISPR array [39]. An RNA guided Cas9 protein can detect the invading DNA or own default segments on spacers (CRISP array) and then cut and subsequently promoted system repairing of these alien regions. However, people have never clarified the mechanisms of critical "detecting" and "repairing" steps, albeit the gene-editing based on Cas9 or similar such as CPf1 has overly prospected and applied. As in the figure (Fig.1g(3), we get a blue and a red-colored DNA segment in the spacers that will be splicing into a series of segments represented by discrete same color segments from $n=1$ to K. (Splicing occurs in all the kingdoms or domains of life; therefore, a DNA segment in the spacer will be unexceptionally subjected with. Such splicing is possibly just from the middle of the segments, which allow some deviations, slightly different from the intron \& exon splicing in eukaryotes. We use colored segments instead of particular sequences since almost all the DNA sequences can follow into this physical pattern.) We called this vertical splicing and marked with an arrow in the figure. The "splicing number" $\mathrm{k}$ which refers to how many levels of splicing will happen depends on the in vivo site situation. The "theoretical frequencies" or the "frequencies which follow the Principle of Least Action" are shown in the formula of (Fig.1g(1)). The actual frequencies of each segment will finally compete out a proper RNA template with proper length, frequency and copy number amount on-site. With this template, Cas9 will make DNA segments to more spacers. Note, this new sequence is no longer the same sequence which originates it, we call it "eigensequence". (This is not the eigenvalue in quantum mechanics; in that discipline, the linear eigenvector or ground state can't be regenerated after mathematical calculation. In bio-systems, it must be continuously regenerated by the real in vivo splicing processes. Here we just partially borrow the

\section{Volume 8 Issue 11, November 2019}




\section{International Journal of Science and Research (IJSR) \\ ISSN: 2319-7064}

ResearchGate Impact Factor (2018): 0.28 | SJIF (2018): 7.426

terminology. Surface tension is the decisive factor in bio-systems, all the "eigenprocess" is for surface tension. Therefore, even two same nucleotide sequences, if their eigencycle is different, then their surface tension or gravitational binding based on the same cell will still be different. The selection of transformation DNA segment in bacteria is based on the surface tension; then the higher surface tension segment enters into the bacteria can be further modified by the immune system.) From the original segment produces new eigensequence into spacers; we call this process "horizontal splicing". As in the figure, suppose blue colored segment can get new eigensequences to be put into more spacers, even a new eigen-eigensequence (can be written down as |eigensequence $\left.\left.e_{\mathrm{n}}\right\rangle\right)$ could possibly be produced from this eigensequence under certain conditions. The red-colored sequence can't get eigensequence, the system will damage it and also remember the pattern for future defense. (We can't simply say that the red-colored segment is invading and blue is belonging to the species. Both blue or red could be invading, just the sequence can produce more eigen sequence will be in a higher chance from their own species. The sequence rests on the spacer is a bio quantum path that needs $\mathrm{z}$ dimensional oscillation to support the vertical splicing; even later transfer to horizontal splicing still needs $\mathrm{z}$ dimensional input; therefore, life always needs sustaining signals.) From here we could see, CRISP/Cas9 or derivatives is not just an immune system, it is actually a real self-evolution-cycling system. Gene(s) are evolved from palindromes, from vertical splicing to horizontal splicing and get eigensequence based on certain in vivo conditions; superposition of the eigensequences is then getting genes. This is a surface tension inversion or gravity binding process; while the binding of gravity of certain gene reaches a certain threshold, then it becomes inheritable. (As in the model, without enough gravity binding, the splicing processes will be fragile, and such a gene will definitely be difficult to be inheritable.) Bio-systems never use the linear sequence like in our sequencing results to judge a gene; they take the frequency energy (or quantum) spectrum of a gene running inside in vivo environment for judgment and issuing functions. Vertical and horizontal splicing from sequence to eigensequence and cycle again if possible is the way a gene in vivo activation and cross-generation evolution. Eigensequence will be the rest (stationary) state of the sequence which issue under certain in vivo genomic environment, so does | eigensequence $\left.\mathrm{n}_{\mathrm{n}}\right\rangle$. We say that CRISP/Cas9 is also an evolutionary system since eigensequences with higher splicing number could possibly establish a system which more complex than the original system which issuing them under the same condition. Such potential if enter into next-generation, could accumulate evolution trend if favorable postnatal conditions are acquired. (We should also note that eigensequence can't be separated from the in vivo environment which produces it. Since the same sequence could get different surface tension to correlate with different surface tension regions. The eigensequence in one cell goes into another cell, will possibly no longer be an eigensequence due to gravity binding, the new cell needs to take its own system to get a new eigensequence based on the input sequence for functioning. This is why bacteria directly uptake of exogenous genetic materials from their surroundings through the cell membrane transformation or plasmid transfer from other bacteria by sex pili are still need the eigensequence cycle to adjust binding before finally be incorporated. From bacteria plasmid transfer till the most advanced species such as human sexual behavior always accompanied by gravity loss as in (Suppl.Exp13); that is for reducing the numbers of eigensequence cycle of biomaterials for the receivers. We also can understand "eigen-" from the Principle of Least Action, bio-systems need "splicing" for all the processes, therefore, "eigen-" means "the least splicing state based on the function". For any required functions, "splicing" can't be too much or too little; therefore, the "least splicing action" which just enough for functioning based on in vivo conditions on-site composes of the "eigen-". Eigensequence is just "most efficient splicing" at the genomic level based on available in vivo conditions. Offspring get better eigensequence not means they could be evolutionarily more advanced than their parents unless the relatively higher surface tension eigensequence can finally bind $\delta \mathrm{K}$ to the "training improvement" level as in (Fig.1a).) From the above facts we could know that people actually have over-prospected for the CRISP/Cas9 based gene editing, besides the widely reported off-targets problems, what put on sequence will continuously subject to certain modifications on-site due to the discrepancy between our input sequence and the real "eigensequence", and also little we can do for it. (Even if we could know the exact RNA splicing sequence of a certain step, while we directly inject RNA with the same theoretical RNA linear sequence to the site, the injected RNA will no longer be eigensequence due to Le Châtelier's effect and diverse other reasons. Dolly sheep is a good example; it is claimed to possess the same genome with the donor. However, due to eigensequence modifications, it is impossible to keep the same genome. It finally only got half of the normal lifespan.) In vivo gene regulation is really a physical process instead of a chemical operation; relative mobility among genes decides the function and is much more important than just chemical nucleotide sequence.

CRISP/Cas9 is the ever known primitive form of the immune system. With evolution advancing, the model of horizontal \& vertical splicing to superimpose eigensequence model is still not changed. The major histocompatibility complex (MHC) is a set of genes that code for cell surface proteins essential for the acquired immunity in vertebrates. The human leukocyte antigen (HLA) complex is a gene complex encoding the MHC proteins in humans, which resides on a $3 \mathrm{Mbp}$ stretch within chromosome 6p21, as in (Fig.1g(4)). Antigen presentation is a vital immune process for $\mathrm{T}$ cell immune response triggering since $\mathrm{T}$ cells recognize only fragmented antigens displayed on cell surfaces. (This is definitely a surface tension inversion process. T lymphocytes claimed to detect a peptide displayed at only $0.1 \%-1 \%$ of the MHC molecules. This detection affinity should come from horizontal \& vertical splicing inversion.) Such antigen presentation or cross-presentation (splicing between MHC I and MHC II) process is actually a process based on the eigensequences. (Start from earlier bacteria, enzymes always like to attack palindromes (higher surface tension part), therefore a high chance of leaving asymmetry characteristic segments (palindromic crest part). To advanced species, lysosomes or even animal digestion still keep such pattern, damage the periodical structures in priority and leave the periodical crest part. No matter genes, proteins, or even cell segments, exogenous characteristic presentations will via surface tension inversion process. Old cell decaying

\section{Volume 8 Issue 11, November 2019}




\section{International Journal of Science and Research (IJSR) \\ ISSN: 2319-7064}

ResearchGate Impact Factor (2018): 0.28 | SJIF (2018): 7.426

surface tension can't issue such inversion; therefore, they will face immune clean.) In a cell, protein molecules of the host's own phenotype or of other biologic entities are continually synthesized and degraded. Each cycle is actually an evolved process of the CRISP/Cas9 eigensequence splicing model we mentioned before. And same with before, this system is an immune as well as an evolutionary system. Just the horizontal splicing processes with enough vertical splicing support have been extended from among-palindromes to amongchromosomes, based on the fact that MHC on human chromosome 6 needs horizontally splicing to other chromosomes for producing the complex. The evidence for splicing in immunity can also come from antigenic peptide splicing from different proteins [42]. For the evolutionary trend, the correlation between HMC \& sexual selection has been observed in mice [43], and the human male-specific region of the $\mathrm{Y}$ chromosome is a result of splicing [44]. (It states "a mosaic of discrete sequence classes" is indeed a process of "splicing", and massive palindromes contain testis genes are quite stronger evidence of the eigensequence model. These reports did not use surface tension models; however, their results are directly transferable.)

Prokaryotic genes lack exon/intron; in eukaryotes, genes need "RNA splicing" which spliceosomes cyclically spins off introns (3'AG-----GU-5') from pre-mRNA and then matured into spliced mRNA as in top of (Fig.1h). In human genomes, the ratio of exons to introns inside genes is average 7.8 to 8.8 [45]. And the intron to intergenic sequence is also near 1:1 across animal species [46]. This means splicing processes are just amplified CRISP/Cas9 model with exon/intron occupy around the spacer/palindrome size. A large gene will then covers a lot of palindromes in scale. Alternative splicing [47], which claimed to potentially result in large numbers of protein isomers is shown in the left bottom of (Fig.1h). In human 95\% of multi-exonic genes are alternatively spliced [48]. Drosophila Dscam is claimed to potentially produce 38,000 isomers [49] (possibly the highest number in literature), and the purpose of alternative splicing is to "increase isomer number for adaptation". Now we can see how eigensequences superposing in right bottom of (Fig.1h), suppose a DNA splicing into " $\mathrm{K}$ " number of exons after removing of introns, then all these exons could be regarded as pro-eigen units with quantum number $\mathrm{n}=\mathrm{k}$; two of them combined together get a few $n=k-2$ eigensequences; similarly, three of them combined together to get a few $n=k-2$ eignsequences, and so on; as the blue colored segments in the figure. In a gene splicing, which eigensequence is finally activated depends on in vivo conditions. If a few genes work together, the eigensequence of each gene can further splice together, and the driving force is still surface tension or gravitational binding. We could then know that among these 38000 potential isomers of Drosophila Dscam, only very small numbers of isomers are produced with enough surface tension. The remaining mRNAs have to sacrifice for the oscillation frequencies or surface tension of those few real isomers. (And the few isomer mRNA templates will possibly still experience horizontal splicing, that is going reside on nearby palindromes and then start the new cycles if conditions are met. The reasons why advanced species need more palindromes just because coded genes really need palindromes as "stationary" structures to functioning under certain conditions.) The purpose of alternative splicing is producing eigensequences for the next steps instead of "increasing isomer number for adaptation". Recursive splicing $[50,51]$ is another type of alternative splicing, for large intron need to be spun off by multi-steps and needs days to finish. Self-splicing occurs for rare introns that form a ribozyme, performing the functions of the spliceosome by RNA alone. tRNA (also tRNA-like) splicing is another rare form of splicing that usually occurs in tRNA. They should be ancient since they are presented in prokaryotes and spliceosomal introns only present in Eukaryotes. Studies from a wide range of organisms show that the intron-exon structure of homologous genes in different organisms can vary widely [52]. More recent studies of entire eukaryotic genomes have now shown that the lengths and density (introns/gene) of introns varies considerably between related species [53]. Intron loss is demonstrated to correlate with gene expression [54,55]. All this evidence has strongly validated our physical splicing model. Quantum superposition is a fluctuation (strengthening and weakening) effect in bio-systems. Exon/intron splicing is such a superposition, as the wave-shaped dotted line in the upper part of (Fig.1h). Diverse epigenetic modifications such as methylation also follow such a pattern. Now we can modulate the surface tension inversion at the genomic level. As shown in the figure, any gene or correlated gene clusters, include Cas 9 or its derivative modified sequences, always via horizontal and vertical splicing to diverse eigen cycles and then express, we can use bio quantum path as follow:

$$
\left\{\begin{array}{c}
x=A_{x} \cos \left(\omega t+\varphi_{x}\right) \\
y=A_{y} \cos \left(\omega t+\varphi_{y}\right) \\
z=S_{z} \cos \left(\omega_{z} t+\varphi_{z}\right),\left\{\left[\begin{array}{l}
s_{z}^{+} \\
S_{z}
\end{array} \mid=k,\left\{\begin{array}{c}
z^{+}=S_{z \text { horiontal }}^{+} \cos \left(\omega_{z} t+\varphi_{z}\right) \\
z^{-}=S_{z_{-} \text {vertical }}^{-} \cos \left(\omega_{z} t+\varphi_{z}\right)
\end{array}\right.\right.\right.
\end{array}\right.
$$

$z^{+}$represents the horizontal splicing and $z^{-}$represents the vertical splicing, no matter is a single gene or the holistic effect of a cluster of genes. The inversion at the genomic level just means the exchange of $z^{+}$and $z^{-}$. E.g. following our previous model, a gene horizontally splices for 7 levels and vertically copies into 36 spacers, then $\left|z^{+}\right| /\left|z^{-}\right|=7: 36$. An event makes this gene inversion, just means $\left|z^{+}\right| /\left|z^{-}\right|$shifts to $36: 7$. (This is a tendency which will impact other correlated things, not a change happened in the right way.) Generally, the horizontal splicing mainly controlled by sequence, shifting within 1-2 levels; however, vertical splicing mainly controlled by the inner environment or surface tension region. Then the more required gene can get more copies, and less required will be fewer copies and finally deleted by the regulation system. Eq.20 just means bio-systems have more surface tension regions; the genome sequence is on an inner surface tension region, then we can use Eq.20 to modulate the inversion of this surface tension inversion. Inner surface tension region like the cell membrane surface tension still possesses its own threshold frequency; this is the reason why diverse mobile genetic elements (MGEs) exist. For MGEs, we need to use relative mobility for modulation. Conventional genetics lacks such a model; therefore, both nonMGEs or MGEs are judged by the linear nucleotide sequence. However, their surface tension should be different even for the same sequence, similar to that of sequence VS eigensequence. Exon/intron strengthening \& weakening follows Le Châtelier's effect; we still can use Le Châtelier's model for one or clusters of correlated genes:

\section{Volume 8 Issue 11, November 2019}




\section{International Journal of Science and Research (IJSR)}

ISSN: 2319-7064

ResearchGate Impact Factor (2018): 0.28 | SJIF (2018): 7.426

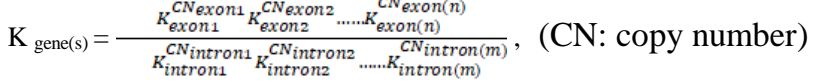

Le Châtelier's Principle can be applied in both surface region and non-surface region, not like most of the physical laws not fit for surface tension region. The difference of the application of this principle in living systems includes multi-surface tension inversions, and for non-living systems, the multisurface tension region still not established; therefore, only irregular molecular inversions.

From genomic to proteomic level, besides the immunological splicing mentioned before [42-44], Protein splicing has been observed in a wide range of organisms, including humans [56]. The gravitational binding of proteins should be generally higher than that of genomic sequences. Protein structures range in size from tens to several thousand amino acids. By convention, a chain under 30 amino acids is often identified as a peptide. By physical size, proteins are classified as nanoparticles, between 1-100 nm. (We should note that such nanoparticles are different from non-living artificial nanoparticles. Protein particles contain very higher Le Châtelier's effect which originates from diverse splicing processes. Non-living nanoparticles lack such characteristics since they don't get any splicing. Some people study the drug delivery by non-living nanoparticles, that's actually quite challenging. Those nanoparticles can pass through the in vivo environment freely means they are non-discernable signals to bio-systems. Therefore, even if they can deliver drugs to a certain locality of the body, these drugs still can't be used. Bio-systems can only utilize Le Châtelier's signals, anything beyond that will be no effect. This is why all the biomaterials need splicing processes.)

For proteins, large aggregates can be formed from subunits. All the repeatable units (such as monomer, dimer, trimer, tetramer, etc.), are functional fluctuation structures and definitely need relevant stationary structures either from self or other proteins for functioning. Peptides folding is driving by surface tension or relative mobility. Vertical splicing reaches certain levels will expand to horizontal splicing; this is a kind of horizontal repeatable units. And while such repeatable units attain a certain level, they will accumulate vertical splicing again. The whole process is then to make "repeatable units" become effective. Structural biology employs techniques such as X-ray crystallography, NMR spectroscopy, and cryo-EM to determine the three-dimensional structure of proteins. Top of (Fig.1i(1)) is a GPCR structure; the bottom shows the quantum mechanics "orbits" and the fundamental crystallography "map" which gets such a structure. With the "map" we can write: $F(h k l)=\sum_{j=1}^{N} f_{j} e^{2 \pi i\left(h x_{j}+k y_{j}+l z_{j}\right)}$ (The structure factor $F_{h k l}$ is a mathematical function describing the amplitude and phase of a wave diffracted from crystal lattice planes characterized by Miller indices $h, k, l$. The sum is over all atoms in the unit cell, $\mathrm{x}_{\mathrm{j}}, \mathrm{y}_{\mathrm{j}}, \mathrm{z}_{\mathrm{j}}$ are the positional coordinates of the $\mathrm{jth}$ atom, $\mathrm{fj}$ is the scattering factor of the jth atom, as in the figure.) Inverse Fourier transform get:

$$
\begin{gathered}
\rho(x y z)=\frac{1}{V} \sum_{h}\left|F(h k l)_{o b s}\right| \cos \left[2 \pi(h x+k y+l z)-\alpha_{h k l}\right] \\
{[\text { ref. } f(x)=A \cos (2 \pi x-\alpha)] \quad(\rho, \text { electronic desity })} \\
\text { or , } \rho(x y z)=\frac{1}{V} \sum_{h} \sum_{k} \sum_{l}\left|F(h k l)_{o b s}\right| e^{\left.-2 \pi i(h x+k y+l z)+i \alpha_{h k l}\right)}
\end{gathered}
$$

$$
R=\frac{\sum_{h k l}\left|F_{h k l}^{o b s}-F_{h k l}^{c a l}\right|}{\sum_{h k l} F_{h k l}^{o b l}} \text { (obs:observation, cal: calculation) }
$$

As mentioned before, the problem of classical quantum mechanics is no consideration of gravity. Albeit structural biology only indirectly uses quantum mechanics for electron diffraction patterns, it still never modulates gravity and surface tension. Whether the structural biology methods such as crystallization, NMR or cryo-EM can mathematically compensate surface tension after getting certain structures is still unknown, if we wish to do so at least diverse relative mobilities is necessary. This discipline needs substantial modifications to integrate their structures with surface tension regions. Suggestions may include setup R of Eq.21 in a range for recovering shifting; or study from the in vivo conditions of the protein or from a series of functional correlated proteins, the latter should be fundamental. Fail to do so means structural biology is still a "dead-and-alive superposition" discipline. GPCR is known as seven-(pass) transmembrane domain receptors (7TM receptors), constitute a large protein family of receptors that detect molecules outside the cell and activate internal signal transduction pathways and cellular responses, as in (Fig.1i(2)). It's a protein superfamily even the size is unclear; it is estimated around 800 genes will code GPCR members. On cell membrane, for a single 7TM the splicing number is 6 (if there are many GPCR on the membrane, the splicing number is 7), then the vertical splicing direction should start from membrane to nuclei successively reduced from $n=6, n=5$ till $n=1$, frequency energy transfer from $n=6$ to $\mathrm{n}-1$ will experience different protein complex, and while this transferring attains a certain threshold, GPCR will extend to other sites on the membrane, this is transfer from the vertical splicing to horizontal splicing. And possibly there is some shifting of structures for GPCR while relocation on the membrane and such shifting will be reflected on the $\mathrm{R}$ range of Eq. 21. This is why resolute protein structures need a series of $\mathrm{R}$ values or other similar parameters; we can't sampling or engineering a series of sample eigenstates; therefore, have to take a series of $\mathrm{R}$ spectrum to understand the eigencycles of proteins. (Fig.1i(3)) shows a report about GPCR signal transfer from NMR [57]. Non-linear relationships have been reported, that possibly could fit the "integer function" $\sqrt[12]{2^{\mathrm{n}-1}}$ For the GPCR functions, it is still not fully understood; we just believe all functions of GPCR proteins are achieved by means of inversion. There is no literature refers to inversion; we infer this conclusion mainly from the surface tension quiddity of life. (Suppl.Exp.14) is performed by avian eggs; however, it can offer some side evidence for GPCR inversion since the avian egg is a big "cell". Nowadays, it is difficult for structural biology to modulate inversion since it's father discipline, quantum mechanics has gotten rid of the inversion model by unitary time, and structural biology methods such as X-ray crystallography, NMR spectroscopy, and cryo-EM are truly challenging in the modulation of the inversion process. I don't know the new model of the photoelectric effect, plus an avian egg AIT injection, and board observations of inversion processes in the biological kingdom are acceptable for people to believe in GPCR and other membrane proteins' inversions.

Proteins not only have their own stationary structures but also will finally superpose on the spinal cord, which is possible via homeobox. A homeobox is a conservative DNA sequence 180

Volume 8 Issue 11, November 2019 


\section{International Journal of Science and Research (IJSR) \\ ISSN: 2319-7064}

ResearchGate Impact Factor (2018): 0.28 | SJIF (2018): 7.426

base pairs long, found within genes that are involved in the regulation of patterns of morphogenesis in animals, fungi and plants [58]. These genes encode homeodomain protein products that are transcription factors sharing a characteristic protein fold structure that binds DNA. As in (Fig.1i(4)), three alpha helixes binding on a DNA chain and the peptide sequence of which is shown in (Fig.1i(5)) with a blue frame. (Homeosis describes the outright replacement of a discrete body part with another body part, e.g. antennapedia replacement of the "antenna" on the head of a fruit fly with legs. The "homeo-" prefix stems from this mutational phenotype, which is frequently observed when these genes are mutated in animals.) Helix 2 and helix 3 form an HTH (helix-turn-helix) structure, where the two alpha helices are connected by a short loop region. The $\mathrm{N}$-terminal two helices of the homeodomain are antiparallel and the longer $\mathrm{C}$-terminal helix is roughly perpendicular to the axes established by the first two. The conventional theory believes that it is this third helix that interacts directly with DNA via a number of hydrogen bonds and hydrophobic interactions, as well as indirect interactions via water molecules, which occur between specific side chains and the exposed bases within the major groove of the DNA [59]. Homeodomain proteins are found in eukaryotes. Through the HTH motif, they share limited sequence similarity and structural similarity to prokaryotic transcription factors. The HTH motif shows some sequence similarity but a similar structure in a wide range of DNA-binding proteins. (One of the principal differences between HTH motifs in these different proteins arises from the stereo-chemical requirement for glycine in the turn which is needed to avoid steric interference of the beta-carbon with the main chain: for cro and repressor proteins the glycine appears to be mandatory, whereas for many of the homeotic and other DNA-binding proteins the requirement is relaxed.) The homeodomain protein motif is highly conserved across vast evolutionary distances. In addition, homeodomains of individual Hox proteins usually exhibit greater similarity to homeodomains in other species than to proteins encoded by adjacent genes within their own Hox clusters. (This means the horizontal splicing among species has been conservative well than the vertical splicing. This is contrary to most genes in which vertical splicing is more conservative than those of the horizontal, which are the characteristics of old genes. HTH motif should be the folding basis of all proteins. So if we want the relative mobility of various proteins, we could use their relative mobility with the HTH motif as the basis.)

In organisms especially vertebrates, the order of the genes on the chromosome (close to one another in clusters) is the same as the expression of the genes in the developing embryo, with the first gene being expressed in the anterior end of the developing organism. For all the species, always the genes located on 3'-end for head and 5'-end for the end of the body. This is called the collinearity. Homeodomain transcription factors typically switch on cascades of other genes. The homeodomain binds DNA in a sequence-specific manner or in the promoter region of their target genes compose as complexes with other transcription factors. Such complexes have higher target specificity than that of a single homeodomain protein. Homeodomains are encoded both by genes of the Hox gene clusters and by other genes throughout the genome. Humans have over 200 homeobox genes of which
39 are Hox genes [58]. From our model, collinearity means horizontal surface tension regions converge on the same vertical splicing. This is the characteristic of the spinal cord. Also, collinearity should come from copy number which originated from the homeodomain binding on a gene network as in (Fig.1i(5)). (Homeodomain can decide the morphology of embryo development; therefore, the gene network they can bind should be thousands of genes. For human beings with $20 \mathrm{~K}$ coded genes, if $1 / 3$ gets expression in embryonic development, then all should be binding by homeodomain. The conservative characteristics of certain genes in evolution are originated from their constant $\mathrm{K}$ binding network size. Homeobox genes are so conservative in evolution; therefore, they should at least bind full spectrum of developmental genes, possibly full genomic spectrum. Here we only draw a very small network as a representation.) Such kind of binding will adjust the harmonic condition of the network, as mentioned before (Suppl.ExpL.6) the frequencies of the same level should be equal, in the figure (Fig.1i(5) for frequency energy we can easily get gene $2_{1}=$ gene $2_{2}$, gene $3_{11}=$ gene $3_{12}$, etc. The protein binding strengthens these harmonic conditions. Also, we could see how $\mathrm{CN}$ (copy number) is initiated in a binding gene network. As in the figure, there present four frequency energy flows such as gene $1 \rightarrow$ gene $2_{1} \rightarrow$ gene $3_{11}$, gene $1 \rightarrow$ gene $2_{2} \rightarrow$ gene $3_{21}$, etc., all could be written into constant $\mathrm{K}$ :

$$
\begin{gathered}
K=\frac{\text { gene frequency energy flow finished state }}{\text { genefrequency energy flow starting state }} \\
=\frac{\text { gene } 2_{1} \text { gene } 3_{11}}{\text { gene } 1} \frac{\text { gene } 2_{1} \text { gene } 3_{12}}{\text { gene } 1} \frac{\text { gene } 2_{2} \text { gene } 3_{21}}{\text { gene } 1} \frac{\text { gene } 2_{2} \text { gene } 3_{22}}{\text { gene } 1} \\
\left.=\frac{\text { gene } 3_{11} \text { gene }{ }_{12} \text { gene } 3_{21} \text { gene } 3_{22} \text { gene } 2_{1}^{2} \text { gene } 2_{2}^{2}}{\text { gene } 1^{4}} \quad \text { (CN of gene } 1 \text { is } 4\right)
\end{gathered}
$$

Now we can clearly see where the $\mathrm{CN}$ of gene 1 and gene 2 are come from, just from the normal binding of homeodomains of the gene network and flow of frequency energy. In a large gene network, Hox genes work in the same way. Within the animal kingdom, Hox genes are present across the bilateria [60] and have also been found in Cnidarian such as sea anemones [61]. Following our surface tension model, any gene network, no matter large or small, always present the inversion state. As in (Fig.1i(5), the frequency energy flow from hierarchy root gene 1 to the terminal genes will be relatively easy, once gene 1 begins to function, all the terminal genes will also get activation. However, gene $2_{1}$ can only activate its downstream gene, even can't activate gene $3_{21}$; if it wants to activate gene 1 , only while all its downstream genes and the parallel such as gene $2_{2}$ get activated, then it can activate gene 1 . This means for any network, positive flow is easy, and negative flow is conditional. Such a difference in frequency energy flow is surface tension that can transfer the frequency energy. Positive flow can happen easily; negative flow requires the spinal cord for adjustment. (The relationship between the "secondary metabolisms" and the "primary metabolisms" is still the copy number. Now we just initiate the concept of network inversion; for details we have to rely on music harmonic later). Brain mosaicism mentioned before, which refers to the higher $\mathrm{CNV}$ should come from the spinal cord based on such kind of surface tension structure. (Fig.1i(6) demonstrates the overlap of Hox genes' expression on embryonic stage spinal cord. CNV actually reflects binding network stability; the stability of any network definitely comes from inversion. No matter a big or a small network, the more it inversion, the more stable it

\section{Volume 8 Issue 11, November 2019}




\section{International Journal of Science and Research (IJSR) \\ ISSN: 2319-7064}

ResearchGate Impact Factor (2018): 0.28 | SJIF (2018): 7.426

is. Retrograde of inversion due to environmental conditions or senility will then retrograde the stability of a network since inversion is the only way for the gravitational binding of the network for functioning.

In bio-systems by size, genomic \& proteomic levels belong to the microcosmic scope, tissues \& organs are in macrocosmic scope, cells are intermediate; they all superposition on the spinal cord. The fundamental function of the spinal cord is to physically reduce the inner resistance and extend effective superposition. At cell levels, eigencells are those with better cytoskeletons (Suppl. Movie 6.1) than other cells. A cytoskeleton is present in the cytoplasm of all cells, including bacteria, and archaea. It is a dynamic network of interlinking protein filaments that extends from the cell nucleus to the membrane. The cytoskeletal systems of different organisms are composed of similar proteins. In eukaryotes, the cytoskeletal matrix is a dynamic structure composed of three main proteins, which are capable of rapid growth or disassembly dependent on the cell's requirements. The structure, function and dynamic behavior of the cytoskeleton can be very different, depending on the organism and cell type [62]. Even within one cell, the cytoskeleton can change through association with other proteins and the previous history of the network [63]. Cytoskeletons compose of the main part of cell surface tension or gravitational binding at this level; they work in a cluster to issue functions instead of individually. However, lack of a detailed model for such superposition at the cell level; we then have to rely on the macrocosmic ancient physical ligaments \& joints training superposition to modulate the cytoskeleton superposition. They are functional counterparts just on a different scale; therefore, the results are transferrable.

A ligament is the fibrous connective tissue that connects bones to other bones. Besides such true ligament, other ligaments in the body include the: Peritoneal ligament: a fold of peritoneum or other membranes. Fetal remnant ligament: the remnants of a fetal tubular structure. Periodontal ligament: a group of fibers that attach the cementum of teeth to the surrounding alveolar bone. Ligaments are similar to tendons and fasciae as they are all made of connective tissue. The differences in them are in the connections that they make: ligaments connect one bone to another bone, tendons connect muscle to bone, and fasciae connect muscles to other muscles, also among or inside organs. All of them should evolve from the cytoskeleton; we just call all of them ligaments based on their common properties. There are three important common characteristics of such ligaments; first, they are notoriously difficult to medically repair. Conventional repairs, including grafting tissue from the patient's body, or placing artificial prostheses to replace ligaments, were quite unsuccessful. Surgical suture which routinely applied for muscles, other tissues or even large nerve fibers, is ineffective for ligaments. (For any ligament torn, what the doctors can do is only remove the torn part under arthroscopy and hope for the body self-repairing, impossible to make any stitching or grafting, etc.) Due to their higher constant $\mathrm{K}$ binding, any repairing will be theoretically difficult; certain claimed technologies such as artificial ligaments or stem cell repairing of ligaments are only stories on publications. (Ligaments bind tissues or bones; that means their repair need come from all the binding parts instead of just ligaments themselves. A genomic sequence can get eigensequence quickly; a ligament to get its eigenligament for repairing have to take a very longer Le Châtelier's time that is impossible for the system; this is the cause of the "impossible" in repairing. Ancient people quite precaution with the protection of ligaments in physical training since they knew from their experience that recovery of a torn ligament is almost impossible. Modern medical sciences have advanced so much nowadays; however, such "impossible" is still not changed. The unrepairable characteristics of ligaments should be applied to the cytoskeleton level. That means cytoskeletons still physically unrepairable; once they damaged or oscillation lower, the only way is immune-clean of the cell.) Second, ligaments are the only anatomical structures in the human body that never get cancer. (CSF is also a structure that will not get cancer; however, CSF is not an anatomical structure; once life is ended, CSF will decay quickly, even faster than RNA in vitro decaying, difficult to exist as an anatomical structure.) Every human tissue includes advanced structures such as the brain, eyes, and blood could get cancer under certain conditions; ligaments are the only exception. This means they have the highest recovering capacity among all human tissues. Cancer needs recovering capability for curing. If this common property applies to the cellular level, we could then understand that the greatest difference between a normal cell and a cancerous cell should be their cytoskeletons. A cell becomes a cancerous cell is first and foremost because of its deformed cytoskeletons or even short of cytoskeletons. Deformed cytoskeleton means their superposition on the spinal cord is "divergent" for various reasons. (From cytoskeleton to fasciae, tendon, ligament and finally superpose on spinal joints to establish a large physical network to shapes the growth, tumor cells just escape the control from the cytoskeleton level. There are a lot of publications to describe the "genomic mutation" of tumor cells. This means without the control of the cytoskeleton, the genome of a cell will induce board mutations. Medical sciences should first find how cytoskeleton regulates claimed oncogenes. Physical processes really govern all chemical or biological processes in a cell.) Third, ligaments or joints at the different parts of the body possess profound correlations, and they finally superpose on the spinal cord. Ordinary people can't voluntarily oscillate their spinal cord; however, ancient ligaments \& joints training systems can make a person doing so. The method of which is actually strengthening above $\mathrm{CN}$ binding. As in (Fig.1j(1)), setup of human hip joints (pointed by green arrows), should joints (blue colored), elbow joints (green and the grey colored), sternum-rib-joint (blue colored), and spinal column joints for opening training as in (Suppl. Movie 7.1-7.7). Such training needs step by step and follows the sequence, every day one thousand repeats for each step; then in 3-5 years, the people can finally oscillate the spinal column as in (Suppl. Movie 7.6). (To oscillate spinal column is easy for lower evolved vertebrates; fishes do this every day. Human spinal cords have bound too much, then need extensive training to recover such a capability. Mammals that come back to sea, such as whales, their directions of oscillating the spinal cord are the same with the human training, orthogonal to the fish oscillation directions). Such training is different from modern gymnastics training. Gymnastics stretch the ligaments to perform various movements, and the purpose is not for opening the spinal cord joints. After retirement, gymnastic athletes still face several problems same with other sportive

\section{Volume 8 Issue 11, November 2019}




\section{International Journal of Science and Research (IJSR) \\ ISSN: 2319-7064}

ResearchGate Impact Factor (2018): 0.28 | SJIF (2018): 7.426

athletes and yet not significantly increase lifespan; these facts just means the stretch lengths of the training ligaments doesn't correspond to the percentage of total cytoskeletons superposed on the spinal cord. In contrast, the opening of the spinal cord is not fully depended on the stretch and elastic of subordinate ligaments. It also needs transferring oscillations to muscles or bones bound by ligaments to activate diverse subordinate cytoskeletons. Without oscillation transferring and only stretch the ligaments can't finally activate spinal joints. From here, we could understand that (Fig.1g(1) (2)) is really fundamental in bio-systems even for ligaments training. For people who finally successfully open the spinal joints in a good oscillation state have all performed at least one million times of each training as in (Suppl. Movie 7.1-7.4), this fact profoundly reveals the oscillation and constant $\mathrm{K}$ copy number quiddity of spinal ligaments to other subordinate ligaments in the body. (Ligaments as the same sort of tissues composed of joints; at different locations of the body, they possess one million training repeats difference; it can only be bound by $\mathrm{CN}$ relationships which show as splicing number during active oscillations. As in (Fig.1h) bottom right, every gene needs splicing to issue functions; the same gene, if get activated, will demonstrate a higher splicing level in spinal ligaments than in subordinate ligaments. This also means the eigensequence needs more cycles to fulfill. In classical Le Châtelier's Principle, subscribes only mean molecular number. However, molecules are not rigid and they take "identical particle" models; we then need to transfer molecular numbers into macrocosmic binding copy numbers.) It could be regarded as the macrocosmic representation of $\mathrm{CN}$ of Hox genes' binding, and also a typical model of gene dominant and recessive. If this common property applies to the cellular level, we could know that cancerous cells should with deformed cytoskeletons and will no longer follow the constant $\mathrm{K}$ binding network. (Note: the number of training movement repeats is not the gene network $\mathrm{CN}$ since each movement has already activated a wide spectrum of genes. Just these two numbers have some correlations. Therefore, an estimated $6 \mathrm{~K}$ copy number on a gene network binding actually needs one million macrocosmic ligament training repeats from each of the different steps for activation. This is just a typical condition for people from 18-35 years old normally join the training, for those who get the physical strike training start from born as in (Suppl. Movie 1.2), only takes around $1 / 4-1 / 5$ of training repeats, however, this degenerative training repetitive number is still quite larger than $6 \mathrm{~K}$. And it is truly difficult for those over a certain age or involves too much sex behavior to finally succeed. Gene dominant or recessive is such a spinal cord binding process.) The conventional theory just explains gene recessive into chemical allele gene shielding; that theory neglects $\mathrm{CN}$ bindings which need to be activated by physical activities. The genomic imprinting [64] percentage should be larger than that reported and belongs to some sort of network binding. Even asexual reproduction and parthenogenesis should include board inversions to assure eigensequences with enough oscillations should enter into the next generations. Otherwise, offspring even with the same nucleotide sequence with that of the previous generation will lose functional genes on a large scale due to the insufficiency of surface tension of physical oscillations.) (Fig.1j(2)(3)) are some maps of Hox gene functions, just habitually not realize and write down $\mathrm{CN}$ by the authors, possibly need some modifications in the future.
(Fig.1j(5)) shows a human spermatozoon; it can also reflect spinal binding profoundly. A spermatozoon generally consists of a flat, disc-shaped head $3.1 \times 5.1 \mu \mathrm{m}$ and a tail $50 \mu \mathrm{m}$ long [65]; the tail flagellates the sperm cell (at about 1-3 $\mathrm{mm} /$ minute in humans) by whipping in an elliptical cone; the mtDNA present in the mid-piece (10-14 spirals of mitochondria surrounding the axial filament in the cytoplasm) which far away from the nuDNA. We can also easily see from the figure, the oscillation frequency of the nuDNA is different from that of mtDNA while swimming. This is possibly the origin of different of $h_{m t}$ and $h_{\text {bio }}$ in frequency energy scales. We refer to spermatozoa here since mitochondria have been used to modulate the spinal functions. (This is possibly the only discussion in conventional biology which refers to the physical functions of the human spinal cord and could offer some clues for $h_{m t}$ modulations.) Let's find some empirical reports; the connection between mitochondria and aging has been verified [66]. Mitochondria concerned with brain \& spinal aging and contributed to ALS (Amyotrophic Lateral Sclerosis) which is a disorder finalized in physical strength [67, 68]. Mitochondria concerned with skeleton muscle aging [69] and motor neuron degeneration [70] have been confirmed. Mitochondria regulate cell apoptosis [71]. All these prove that mitochondria deeply correlate with aging, which means they definitely participate in functional gene network binding. As mentioned before, $h_{m t}=\frac{1}{10} h_{b i o}$, their participation of binding should at least via frequency energy or even splicing. Also, the mitochondria network with neurogenesis in the spinal cord has been validated [72]; this correlates them with thinking and brain functions. CSF involved with multiple sclerosis [73,74]. Mitochondria relate to intestinal development [75]. Mitochondrial DNA modulates telomere length [76]. Vigorous phosphotransfer between mitochondria and nucleus has been observed [77]. Mitochondria direct impact on brain function and cognition [78] by dynamically undergoes shape-changing. All these could be evidence for splicing, and strong evidence of deep splicing can be found on mitochondria structures. In humans, a cell contains around $10^{3}-10^{4}$ mitochondria, each with $2-10$ copies of mtDNA composed of 16,569 base pairs of mitochondrial DNA encode for only 37 genes [79], which just produce 13 proteins, 22 tRNAs and two rRNAs. In the cells of extant organisms, the proteins present in the mitochondria (around 1500 different types in mammals) are coded from nuclear DNA (most of them are of prokaryotic origin) [80]. The majority parts of $\mathrm{mt}$-proteins are splicing from nuclear is indisputable evidence of stronger splicing among mtDNA and nuDNA, even shielded by the fact that the coding system of mtDNA is different from that of nuDNA. The strength of splicing which induces a higher percentage of mt-proteins to come from nuDNA is powerful enough to impact reproduction. Paternally inherited mitochondria have been reported in some insects such as fruit flies [81], honeybees [82], and periodical cicadas [83]. And also have been found in sheep [84], cloned cattle [85], included a single case in a human male [86]. These reports mean that albeit there is a distance between mtDNA and nuDNA in spermatozoa, after instilling genomic DNA to the ovum, mtDNA in the midpiece region will still enter into the gamete later by certain mechanisms. The detection of parental mtDNA [86] is claimed as an abnormal condition;

\section{Volume 8 Issue 11, November 2019}




\section{International Journal of Science and Research (IJSR) \\ ISSN: 2319-7064}

ResearchGate Impact Factor (2018): 0.28 | SJIF (2018): 7.426

however, even in human beings, it can be claimed as an accident; we actually can't neglect the fact that paternal mtDNA inheritance has been presented in the animal kingdom for quite a long time. There are also reports claimed that paternal mtDNA could be detected in the 4 to 8 -cell stage [87] of some abnormal embryos but never sustained untill infants after born [88]. If human beings have really evolved some "clearance mechanisms" for the "accidentally" infused mtDNA, then the mechanisms should be engensequence model due to high splicing between mtDNA and nuDNA. And such a clearance manner possibly can't assure $100 \%$ clearance under any conditions. If such a "clearance mechanism" is not as general as claimed, then the mtDNA "accidentally" entered into the gamete will very likely to present into the spinal cord. And in the spinal cord, this paternal mtDNA will bind maternal mtDNA or even nuDNA if some conditions could be met. Like in the ancient training, other joints need one million repeats to activate spinal joints; we actually don't know whether the "abnormal" report of the human paternal mtDNA case [86] is due to a lack of proper physical stimuli. (In a binding gene network, if upstream genes can't get downstream genes' signals for a certain period, it will then retrograde or inverse. Like the ancient training, spinal joints need one million repetitive signals from other joints for stimuli. The person in the case probably just needs physical activation to be "normal". Alpha system has been widely presented in animal societies. Alpha males should correlate with paternal mtDNA transferring profoundly, or mtDNA can tighten $\delta \mathrm{k}$ under certain postnatal conditions. Even only consider the evolutionary trend, mtDNA will either function for both parents or retrograde to cease to function for any single-sided parent, less likely to work only from one parent in the long term. Such a pattern is evolutionarily unstable, which quite difficult for survival. From this fact, we can understand mtDNA enter into the gamete is still a surface tension problem. The distance between the mtDNA and nuDNA covers by many surface tension regions; mtDNA needs relay inversions to pass all these surface tension regions to enter the gamete, and this relay inversion manner correlates with the long journey. Most of the mtDNA did not get enough capability from the long journey to activate their inversion capability to pass these surface tension regions, only a small number of mtDNA can and then get alpha males. It's the second selection after the nuDNA success. Also, we could understand, both mtDNA and nuDNA contribute to the surface tension or gravitational binding of the gamete and embryonic development profoundly.)

Summary in this section, life is a multi-surface tension region system, and inversion is the basis of the gravitational binding for each surface tension region. Molecular structures follow K $=\gamma / \mathrm{g}$. All the surface tension regions have their own Einstein's threshold frequencies that can be modulated by bio quantum paths. The most efficient energy transferring follows the Principle of Least Action, and the least transfer energy is the inversion frequency energy combinations of all the surface tension regions; therefore, proper outside physical strike will induce a relay inversion of each surface tension region. Biological processes discussed in this section, such as NuDNA \& mtDNA interaction, gene horizontal \& vertical splicing, membrane protein functions, and Hox gene binding network, etc., and all other processes which not discussed, are all need to be modulated by surface tension inversion. Classical quantum mechanics is a surface tension discipline that only takes wave function for one layer of surface tension and lacks commutation time or inversion; bio-systems need a multi-layer wave function to modulate the CSFs and other structures, each with inversion.

\section{Upgrade Schrödinger wave function into "repeatability fluctuation", modulate of CSF wave function by bio quantum path $\mathrm{z}$ dimensional fluctuation superposition}

Water clocks are one of the oldest time-measuring instruments in which time is measured by the cascade drops of liquid where the final amount calibrated as time. They are the most accurate and commonly used timekeeping devices for millennia until replaced by more precise pendulum clocks. As in the top of (Fig. 1k), we put water in a container then drop it. The time of drop out one container of water could be calibration as time. If we add an overflow on the container, the time becomes more accurate since the overflow drops stable the level of water surface under certain environmental conditions. If we cascade a few of such container units, then the time becomes more and more accurate. (The cascade number is not unlimited. Ancient artifacts generally composed of 4-7 cascades, at the final container, took a floating device for time calibration since it is the most stable one among a series of containers.) Not only liquid, but electronic circuits are also following similar rules. Nearby the water clock is a simple LC circuit with simple harmonic oscillation $\omega$, and f wrote below. (Add R (resistance) into LC just makes the simple harmonic motion into a damped oscillation, will not change the cascade effect too much.) The water clock is an open system that can converge periods or oscillation frequency by overflowing. Not just for periods, other parameters such as concentration or even physical force can converge. (One or more chemicals into the first container without stir, then concentration accuracy or mixture uniformity will converge in the final container. And such converge is not due to human stir in the container to make solvent evenly distributed, it is due to entangled environmental conditions combined together via surface tension of the water gradually converge the simple harmonic motions of the system. In one container, water surface tension from a large water body flows to small water drops, surface tension region percentage of the drops increased. However, among all surface tension percentage increased water drops, the drops from the overflow tube still contribute to the stability of the drops to the next container; this is the "anti-unitary" or "turnover" process we mentioned before. This is the general mechanism of "excretion" or splicing; just something leaves to increase the surface tension region percentage of another thing. If we watch carefully, the formation of a water drop still experiences a lot of inversions before finally establishes the one layer surface tension region of a water drop.) Albeit only one layer of surface tension region, it has already shown Le Châtelier's effects. The Le Châtelier's effects of living beings will quite stronger than this due to multi-surface tension regions have been integrated into; however, the mechanisms are the same. The top right of (Fig. 1k) shows some levels of the human fluid system; it can be regarded as a water system increased to higher percentages of surface tension region. From gut to CSF (cerebrospinal fluid) composed cascade of "anti-unitary"

\section{Volume 8 Issue 11, November 2019}




\section{International Journal of Science and Research (IJSR) \\ ISSN: 2319-7064}

ResearchGate Impact Factor (2018): 0.28 | SJIF (2018): 7.426

converging oscillations. (For the human spinal column, the central oscillation is located in L4 (4th Lumbar Vertebra), which directly opposite with the navel button. In the developmental process, umbilical blood comes from navel to transfer oscillations to somite directly start from here. We can see an experiment. (Suppl. Movie 8.0) shows a chicken after releasing of CSF and not fixed it for a long time; then it will uncontrollably struggle to the death. This is not due to hurt the nervous system since that only can make a chicken lame or crippled and not death. (For a human, the daily CSF production is $500 \mathrm{ml}$, and a spinal tap can get only $1-10 \mathrm{ml} \mathrm{CSF}$, around $1 \%$ of the total amount. This means for a chicken only release $1 \%$ of the CSF is enough to kill it if not handling properly. In contrast, releasing blood to kill a chicken needs at least $70 \%$ of the total amount. This is due to the higher physical binding of CSF than that of the blood.) For human beings after sampling of CSF, the patient needs to strictly maintain a supine posture for at least two hours and not turn the body. However, even under such medical precautions, implications such as post-spinal headache with nausea, etc., are quite usual. (Right after CSF sampling, if a human still takes stronger physical activities like the chicken, then fate will be the same. The more struggle, the more approach to death.) This means that CSF sampling is a physical bio-inertia, surface tension, or lifespan decaying process; without absolute necessity, we really need to avoid it. The condition for other vertebrates is the same; actually, not just for CSF, for any binding hierarchy network, the repairing of the damaged upstream structure needs compensation from all the downstream structures. (The bacterium is a simple life; plasmid genes are those can bind a larger network from the genome while activated; therefore, removing of a plasmid is difficult to be repaired by the binding network, similar to removing the CSF from an animal. If a bacterium wants to fix a plasmid gene into a genomic gene, it needs a long term evolution in which the whole binding gene network gets the opportunity for a stable inversion. The reason we can apply plasmid for editing is also the bacteria themselves need a quite long Le Châtelier's time to repair the upstream genes on a binding network; therefore, difficult to regulate human-edited genes into the system optimum state in a short term; however, diverse endosomes will produce for compensating the system regulation failure. Inclusion bodies in prokaryotes are such kind of primitive "tumors".) From the CSF experiment, we can see the inseparability of the motion and "stationary". One animal is more "stationary" than another animal; it just means it has more potential to keep CSF or tighten $\delta \mathrm{k}$ under the same environmental stress conditions than another animal, or the surface tension regions superposition on its spinal cord possesses a larger potential than another animal. For any vertebrates at an early stage, the amplitudes of superposition inversion from every part of their bodies on the spinal cords are quite larger, and such amplitudes decrease with age, while such amplitudes decay to certain thresholds or ineffective superposition attains certain percentage, death will happen. For a young man and a dying old man, their spinal cords are only different on superposition strength from a physical point of view; definitely, such decaying could possibly be accompanied by certain clinical detectable symptoms such as syringomyelia, spinal disc herniation, etc., under certain conditions.
The human CSF is a very clear, colorless body fluid, about $125 \mathrm{~mL}$ of CSF at any one time, and about $500 \mathrm{~mL}$ is generated every day with an exchange rate of 4-5 times a day, quite higher than that of the blood system. CSF contains approximately $0.3 \%$ plasma proteins, or approximately 15 to $40 \mathrm{mg} / \mathrm{dL}$, depending on sampling site [89]. In general, globular proteins and albumin are in lower concentration in ventricular CSF compared to lumbar or cisternal fluid [90]. This continuous flow into the venous system dilutes the concentration of larger, lipid-insoluble molecules penetrating the brain and CSF [91]. These facts demonstrate our CSF inversion model ((Fig. h,g) of (suppl. ExpL. 4)). The transparency will gradually be opacification due to senility. CSF pressure is a dynamic pressure system different in range with a blood pressure of $80 / 120 \mathrm{mmHG}$, around $10-18 \mathrm{cmH}_{2} \mathrm{O}$ (8-15 mmHg) with the patient lying on the side and 20-30 $\mathrm{cmH}_{2} \mathrm{O}$ with the patient sitting up. CSF pressure determines intracranial pressure with physiological values ranging between 3 and $4 \mathrm{mmHg}$ before the age of one year. CSF moves in a single outward direction from the ventricles, but multi-directionally in the subarachnoid space, physically oscillating with the pressure waves of blood vessels [92]. Some authors dispute this, posing that there is no unidirectional CSF circulation, but cardiac cycle-dependent bi-directional systolic-diastolic to-and-fro craniospinal CSF movements [93]. (Suppl. Movie 8.1) is an MRI image of blood pulse superpose on the brain and CSF region. (Suppl. Movie 8.2) demonstrates one period of CSF movement. The bottom of (Fig.1k) is an animal comparable developmental map; the embryonic stages of most animals generally morphologically show some characteristics of the previous evolutionary species. E.g., a human embryo will still present a gill slit stage like a fish, or a long tail like a reptile, etc. However, we should understand the difference between the human embryonic fishlike stage and a real non-evolved fish. The stationary superposition structures of fishes are their own spinal cords, and the superposition structures of fishlike human embryos are their mother's spinal cords. Different in stationary structures means quite a different surface tension structure; make these two similar shaped organisms totally different; here, distinctively shows surface tension regions govern the genomic and other expressions.

We have discussed some limits of quantum mechanics in bio-systems and still not refer to the definition of "wave function". In quantum physics, a wave function $\Psi$ is claimed as a function of the degrees of freedom corresponding to some maximal set of commutation observables. The bottom of (Fig. 1i(1) is the dynamic shape of some of the electronic wave functions. By convention, the classical wave function only refers to the isolated system. As mentioned before, it is first a regenerative wave-particle duality; "regenerative" has to happen in an open system since "regenerative" means an interacting system with our research target and the full environment; "repeatability" likes a Gaussian curve in the right bottom of (Fig.1f), the more repeats of a certain event based on the interacting system, the more close to this curve based on that system, it still only refers to part of the environment, so we called it "repeatability fluctuation", means a fluctuation induced by repeatability based on a certain interacting system. "Repeatability fluctuation superposed on 


\section{International Journal of Science and Research (IJSR) \\ ISSN: 2319-7064}

ResearchGate Impact Factor (2018): 0.28 | SJIF (2018): 7.426

the certain system" is the wave function definition. Now we first see the wave function in the human CSF, then back to understand the non-living wave function. As in (Fig.2a), CSF is a multi-surface tension system; we then can use multi-layer of bio quantum path $\mathrm{z}$ dimensional fluctuation to modulate it.
Each layer of surface tension region of CSF can be given as before:

$$
C S_{\text {oscillation }}\left\{\begin{aligned}
x & =A_{x} \cos \left(\omega t+\varphi_{x}\right) \\
y & =A_{y} \cos \left(\omega t+\varphi_{y}\right) \\
\left\{\left|S_{z}^{+}\right| /\left|S_{z}^{-}\right|\right. & =k_{i},\left\{\begin{array}{l}
z^{+}=S_{z}^{+} \cos \left(\omega_{z} t+\varphi_{z}\right) \\
z^{-}=S_{z}^{-} \cos \left(\omega_{z} t+\varphi_{z}\right)
\end{array}\right.
\end{aligned}\right.
$$

Figure 2. Abiogenesis of the multi-surface tension region life and converging superposition, define the concept of "quantum" into bio quantum path

a)

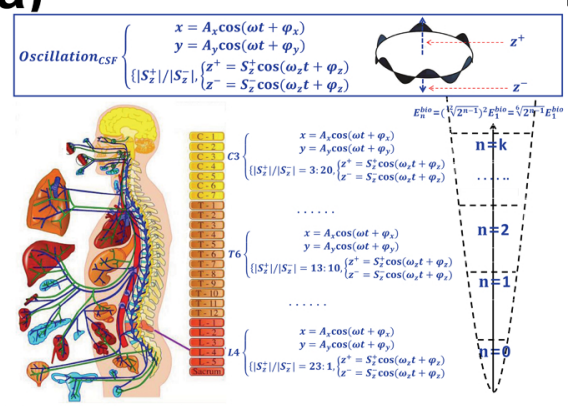

d)

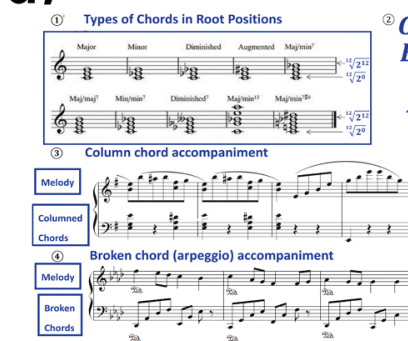

g)

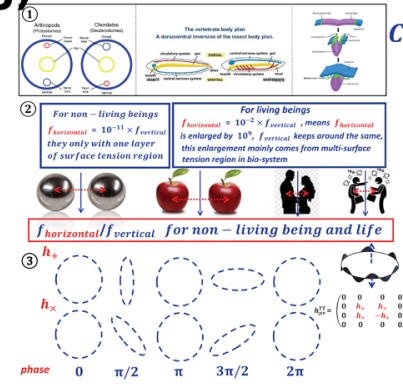

b)

e)
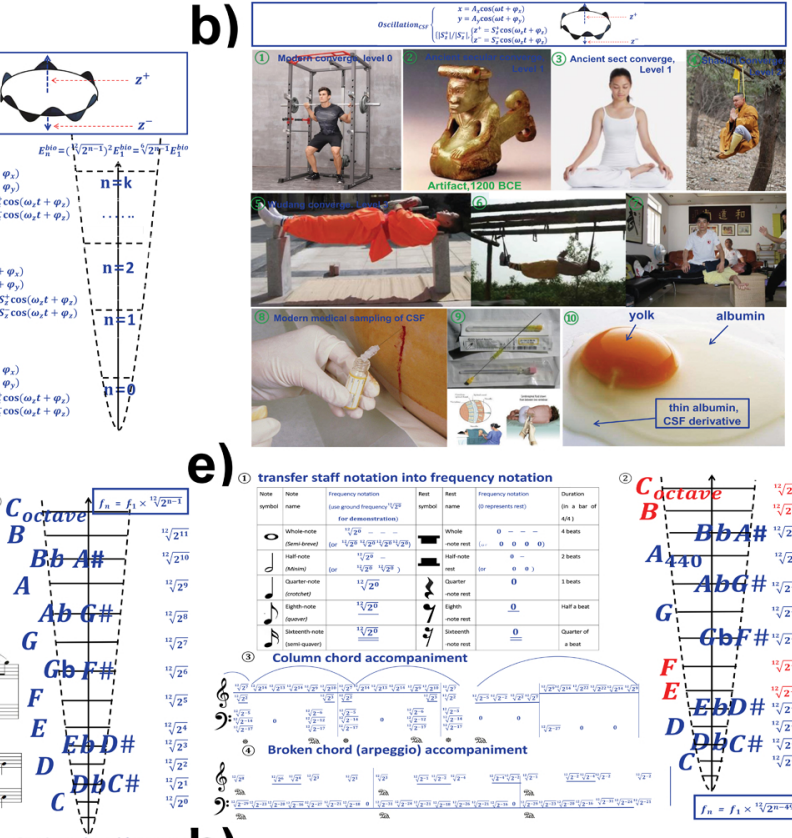

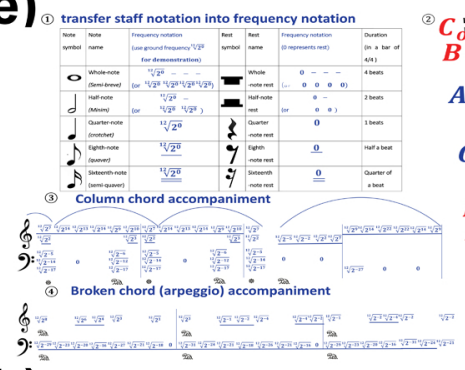

h)

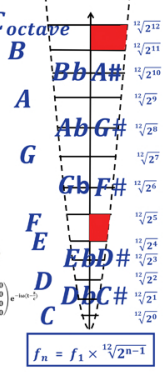

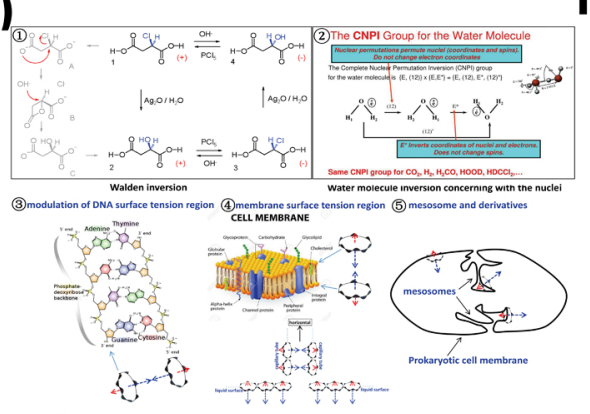

c)

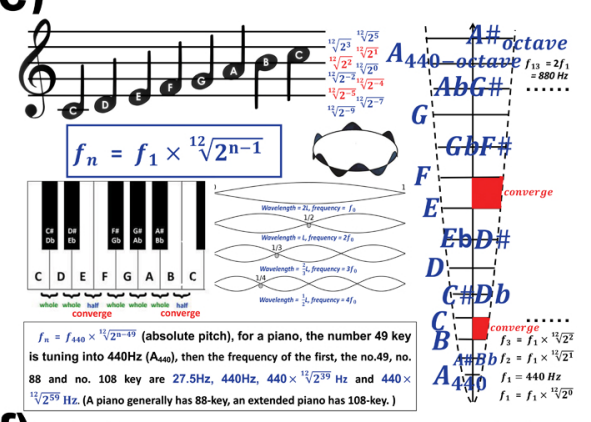

a) modulation of CSF wave function by bio quantum path $\mathrm{z}$ dimensional fluctuation superposition

b) macrocosmic modern and ancient physical converging training at the somatic level

c) Chu's constant and musical tonality with frequency converge

d) conventional musical staff notation, melody with different accompaniment combinations

e) frequency notation, inversion number and quantum number in eigen part of Schrödinger wave function and Einstein's gravitational waves

f) inversion modulated by frequency notation, elementary "memory" model

g) horizontal and vertical gravity difference between living beings and non-living beings is due to different surface tension region percentage, the existing of gravity is also due to the universal existing of surface tension region for all the existence

h) surface tension region inversion for small molecular, large molecule, cell membrane, and cell membrane folding modulated by bio quantum path $z$ dimensional fluctuation for earlier evolution i) life is abiogenesis from a whirlpool by surface tension region inversion and then increase the number of surface tension regions by folding, the standard definition of quantum by the model of bio quantum path in bio-systems

Here $\left|S_{z}^{+}\right| /\left|S_{z}^{-}\right|$represents one layer of surface tension region, there are 24 vertebrae in spinal joints, and the center is L4, roughly regard each vertebra are in equal length, there are total 23 vertebrae above $\mathrm{L} 4$, the surface tension of the number of vertebrae above L4 will be $\left|S_{z}^{+}\right| /\left|S_{z}^{-}\right|=|23-\mathrm{n}| /|\mathrm{n}|, \mathrm{L} 4, \mathrm{~T} 6$ and C3 have been given in the figure, all other vertebrae can be written in the same way just with different ratios. Each layer also continuously composed of 12 layers of subordinate bio quantum paths, and so on, at least $12 \times 24=288$ layers of bio quantum paths. (For the exact number of surface tension regions, we can see embryonic developmental cleavage. Each come from these 3 germ layers. In (Fig.2a), we just neglect all the differences and roughly modulate them as equal. We also note that cleavage is never synchronized with genomic shifting, which means each gene has potentially controlled by more than one surface tension region. The $10^{9}$ levels of cleavage is a surface tension region. Zebrafish embryos under a microscope, just 3 hours can reach the "1k-cell stage"; after this stage, how many cleavages can finally attain is still unknown. Human embryonic rapid cleavage takes $12 \mathrm{~h}$ and results in a 32-cell morula; humans should have much more numbers of surface tension regions than a zebrafish, so we roughly estimate as $32 \mathrm{k}$ surface tension regions; they superimpose to the next stages and fuse into 3 germ layers. The further development of these 3 germ layers actually relies on the superposition of the previous $32 \mathrm{k}$ surface tension. Human spinal cervical, thoracic, and lumbar vertebral numbers also gravitational binding in bio-systems come from the above $32 \mathrm{~K}$ surface tension regions. If the estimation of $32 \mathrm{~K}$ surface tension region is correct, that means each surface tension can bind around $3 \times 10^{3}$ levels of gravitational waves. Humans 


\section{International Journal of Science and Research (IJSR) \\ ISSN: 2319-7064}

ResearchGate Impact Factor (2018): 0.28 | SJIF (2018): 7.426

defeat other species on Earth only due to CSF wave function binding capacity; in the future, if another species that more advanced than humans present on Earth, they still should rely on CSF binding capacity to defeat our Homo sapiens.) As mentioned before, for any surface tension region, the least frequency energy is the inversion energy. We have taken one single layer of surface tension region to modulate the photoelectric effect and bio-membrane before, now the condition with the multi-surface tension region of CSF as in (Fig.2a) is the same, all the bio quantum inside CSF body still transfer energy by means of inversion. Inversion is the least frequency energy transfer manner in the CSF. Besides vertical, there are still horizontal transferring to the attached body structures, all controlled by diverse CSF surface tension ratio levels. For the above ratios, even if temporarily shifted by various conditions, they will recover back later; they only shift with age. E.g., the L4 bio quantum path is $23: 1$, at old age, this inversion ratio could gradually retrograde into $22: 1$ or other ratios since the surface tension region percentage in the body is reduced. We are tired or need to sleep, still because of inversion. CSF regulates all the horizontal \& vertical surface tension shifting, while some parts fail to inversion by the least frequency energy transferring manner, we'll feel tired, sleep just compensate for the correct inversions by the CSF. At old age, sleepy time will gradually be reduced since our CSF inversion capability has been retrograded. (Suppl. Movie 8.3) is the simulation of the whole process; the white membrane represents the surface tension barrier (it can't be seen anatomically, just CSF inner surface tension region); CSF contents oscillate between two barriers, part stays oscillation inside and transfer horizontal surface tension, part pass the barrier by inversion and goes to the next vertebra. This is a vertical relay inversion. Now we can clearly see what "repeatability fluctuation" means, the contents repetitively oscillate between two barriers, this is repeatability, then fluctuate and some inversion out. Only parts are inversing out since the $\mathrm{z}$ dimensional inversion of a bio quantum path still has $\mathrm{x}, \mathrm{y}$ dimension to correlated each other by Le Châtelier's effect. The whole process entangles with the environment and can't be isolated. (Here just show the standard model of one layer of bio quantum path inversion, in real condition, possibly even in one vertebra, there are hundreds of layers of bio quantum paths. This is the process of the Principle of Least Action.) There are diverse relay inversions at different levels of the body, like the cell in (Fig.n) of (Suppl.ExpL.4), all need to be controlled by the CSF. For plants, there are no circulation system and spinal cord; however, inversion is still very general, especially at the photosynthesis and the reproduction structures. For the very general inversion process in surface tension region, besides various observable evidence in bio-systems plus (Suppl.Exp.14), even just by the pure math from the Newtonian times, we can find evidence. Simple harmonic motion is a cosine function; its derivative is the following:

$$
\frac{d}{d x} \cos (x)=\lim _{\Delta x \rightarrow 0} \frac{\cos (x+\Delta x)-\cos (x)}{\Delta x}=-\sin (x)
$$

The derivative of a function of a real variable measures the sensitivity to change of the function value (output value) with respect to a change in its argument (input value). Now the derivative of a cosine function is $-\sin (x)$, there is $\frac{\pi}{2}$ phase angle between $\cos (\mathrm{x})$ and $\sin (\mathrm{x})$, and there is also a minus symbol, then it's a standard symmetric inversion. This means the "least action" change of any cosine function oscillation is its inversion; this simple derivative equation perfectly interpreted the Principle of Least Action with simple harmonic motion oscillation. For the inversion ratio, most of the harmonic motions we see are symmetric on $\mathrm{z}$ dimension, that means $\mathrm{z}+/ \mathrm{z}^{-}=1: 1$, now in $\mathrm{CSF}$, different inversion ratios just mean different degrees of splicing between two-phase, they combined together by Le Châtelier' effect and composed all the surface tension pool of the whole body. For CSF, major bio quantum path inversions happen at the spinal joint regions, at the spinal bone regions, there are still full of inversions, just strength will be lower than those at the joint regions. At the joint regions, they can permeate the whole body and form diverse CSF derivatives. Such permeate is by means of inversion. The concept of diverse blood-brain barrier derivatives are actually incomplete, CSF and derivatives as one (composited) phase must separate with another (composited) phase at every part of the body. Blood-brain barrier just one mechanism we explain the separation of blood with CSF at the brain region, however, at every part of the body, CSF and derivatives must separate with another phase to keep the surface tension region or inversion capacity, once the phase contact surface disappeared, the bio quantum path inversion capacity of that contact surface will also disappear. For this reason, the blood-brain barrier (BBB) and diverse derivatives are not "barriers", or they are not present there to barrier something; they just present there to increase the inversion of certain locations. The more "barriers" we can anatomically observe in the brain means the more inversion capacity at that location. Diabetes is such a disorder due to insufficient surface tension inversion capability to separate the blood sugar phase.

In classical physics, there is a story of "Maxwell's demon" that came from a thought experiment of James Clerk Maxwell. Now we can see, CSF does play the role of "Maxwell's demon" inside the human body. (The capability of this "Maxwell's demon" to control molecules in and out of a cell membrane still comes from inversion; holistic system inversion control a single cell membrane's inversion, and the single cell membrane also contributes to the holistic inversion. This "Maxwell's demon" only can work for a lifespan; when "he" died, life will be ended. CSF tap is sampling this "Maxwell's demon", will threaten life right away if not handled properly.) As mentioned before, quantum mechanics is a surface tension discipline. We can't simply say that Schrödinger's wave function only refers to one layer of surface tension, and the CSF wave function refers to the multi-surface tension regions. As in (Fig. 2a), each different $|z+| /|z-|$ composes a leading surface tension region which controls a lot of subordinate surface tension regions. For the square of these ratios $\left|z^{+}\right|^{2} /\left|z^{-}\right|^{2}$, if we fixed $z^{-}$and only leaves $z^{+}$, then $\left|z^{+}\right|^{2}$ becomes the "probability amplitude" in quantum mechanics. Probability amplitudes provide a relationship between the wave function (or, more generally, of a quantum state vector) of a system and the results of observations of that system. This means quantum mechanics only fit for evenly distributed surface tension region; under this condition with unitary time evolution and diverse rules, it can neglect gravity, and energy is conservative. Also, the wave function $\psi$ must be continuous and all its partial derivatives $(\partial \psi / \partial \mathrm{x}, \partial \psi / \partial \mathrm{y}$, etc.)

\section{Volume 8 Issue 11, November 2019}




\section{International Journal of Science and Research (IJSR) \\ ISSN: 2319-7064}

ResearchGate Impact Factor (2018): 0.28 | SJIF (2018): 7.426

must also be continuous. And since $\int \Psi^{*} \Psi d \tau$ is the probability density, it must be single-valued. In the real-world, quantum mechanics works well for most of the single element atomic emission spectrums. At such a state, all the "orbits" are a concentric sphere or asymmetric; then each surface tension is theoretically evenly distributed. However, once these elements become compounds, or even a single element has enough molecular folding, it is quite challenging to work again. Bio-systems possess vast folding and unevenly distributed surface tension region; therefore, energy is not conservative. Bio-systems are actually surface tension region conservative systems (the number of surface tension region is relatively stable for recovering capacity) and not energy conservative systems. In an ideal condition, inversion frequency energy for the same gravitational binding tends to conservative. The concept of Schrödinger wave function is transferrable, just need to integrate with gravity, albeit Schrödinger equation can't work in bio-systems. And CSF wave function is not single-valued due to inversion even for a single-celled species. (Suppl. Movie 6.0) in (Suppl.ExpL.7) explains the most simplified bio-wave function.

The definition of the wave function "repeatability fluctuation" also fits for non-living beings. The right figure of (Suppl. ExpL. 6) shows electrons pass through the slit(s) then get diffraction patterns on the screen, no matter electron is passing one by one in certain intervals, or pass by any combinations of certain numbers of electrons, once any combinations passing by with enough repeatability, then the diffraction will happen. Now the slit is a controlled "repeatability unit", and all these are in the non-isolated environment, thus "repeatability" can fluctuate. (This experiment also can be performed on water or oil surface, which means quantum mechanics is actually a surface tension discipline.) It is obvious that any events which can reduce the "repeatability" will decay the diffraction and which can enhance the "repeatability" will then strengthen the diffraction. Some people took the double-slit to try to show Copenhagen interpretation that "observation or human consciousness causes collapse of the wave function"; we should know the factor decay the pattern is not the "observation" or "consciousness", it is whether such an event else can damage the "repeatability", if damage then the diffraction pattern disappear, if not, no matter how we observe and how our "consciousness" works, the pattern is there. The quiddity of the Schrödinger wave function is "regenerative repeatability fluctuation" instead of other claims. .

To integrate gravity into wave function, we need to understand the gravity in bio-systems is surface tension polarization gravity, possibly some difference with the conventional gravity. In conventional Newtonian mechanics, surface tension is in a neglected corner, and the orthodox "rigid body" model even excludes surface tension region. However, it is easy to measure; just a simple capillary tube can get $\gamma=\frac{\rho g h}{2 r}$, albeit multi-surface tension is challenging to measure. We can regard it as the binding part of Einstein's gravitational waves, yet Einstein still followed Newtonian tradition rarely wrote down surface tension. For Einstein's emission gravitational waves, following his describe under approximation conditions, metric tensor $g_{\mu \nu}$ can be written as
Minkowski metric $\eta_{\mu \nu}$ and a tiny perturbation $h_{\mu \nu}$ for spacetime geometry: $g_{\mu \nu}=\eta_{\mu \nu}+h_{\mu \nu}$, input into Einstein's field equation in the vacuum: $\left(\nabla^{2}-\frac{1}{c^{2}} \frac{\partial^{2}}{\partial t^{2}}\right) h_{\mu \nu}=0$, then get the quadrupole of gravitational waves:

$$
h_{\mu \nu}^{T T}=\left(\begin{array}{cccc}
0 & 0 & 0 & 0 \\
0 & h_{+} & h_{\times} & 0 \\
0 & h_{\times} & -h_{+} & 0 \\
0 & 0 & 0 & 0
\end{array}\right) e^{-i \omega\left(t-\frac{z}{c}\right)}
$$

This equation means transverse spacetime distortions induced by the acceleration of mass can be propagated at the speed of light like ripples. Mass and energy can be transferring each other, and the more acceleration of mass, the more gravitational waves. There are two polarizations, plus (+) and cross $(X)$ in the quadrupole polarization equation. (Take a ring of test masses with radius $\mathrm{L}$ on $\mathrm{xy}$ plane responding to wave propagating along $\mathrm{z}$, space stretches and squeezes while gravitational waves transverse this ring and it will become an ellipse with the major axis \& minor axis as:

" $L+\delta L$ " \& " $L-\delta L$ ", respectively; then $h_{+}(t)=\delta L / L$ The direction of distortions $\delta L / L$ is orthogonal with gravitational waves' propagation vector.) We can call the $\delta L / L$ as polarization rate. Einstein's gravitational waves modified Newtonian gravitational forces into a transferring speed of light instead of unlimited. The emission part of gravitational waves has partially verified by LIGO for the lower limit range; albeit the other ranges and the existing of graviton are still unknown, it has validated that gravitational waves do transfer at the speed of light. However, in Einstein's theory, the polarization rate $\delta L / L<10^{-21}$, it is too lower since Einstein is too cautious to beyond the Newtonian results and doesn't notice the surface tension region. Once the emission gravitational waves enter into the surface tension region, $\delta L / L$ greatly enlarged, $h_{+}$and $-h_{+}$in the quadrupole becomes the $\mathrm{z}+$ and $\mathrm{z}$ - fluctuation of bio quantum path, and the item $\mathrm{t}-\mathrm{z} / \mathrm{c}$ becomes the oscillation amplitude (ref. Eq. 17, Eq.12). (The least scale of $\delta \mathrm{L}$ in bio-system should $>h_{m t}, h_{\text {bio }}$.) This is one layer of surface tension region with $\pm \delta L / L$. As mentioned before, CSF is a multi-surface tension liquid, with each layer " $\pm \delta L_{i} / L_{i}$ " writes into bio quantum path $\mathrm{z}$ dimensional fluctuation, we then get the CSF wave function of (Fig.2a); Einstein's quadrupoles “ $\pm \delta L_{i} / L_{i}$ " do correlate with the movements of bundles of materials, just now these materials become in vivo gravitational binding structures. (In Einstein's theory, the relative speed of $\delta L$ to $L$ is very high, that is due to no surface tension regions. Now in surface tension regions, once the light speed gravitational wave emission part attained, the speed of the polarization response $\delta L / L$ of a certain structure becomes very lower. In bio-systems, this relative mobile speed of $\delta L$ to $L$ is the binding gravitational waves and should be equal to the $R_{\text {mobiliy }}$ we discussed before. In bio-systems, it is estimated around $200 \mathrm{~m} / \mathrm{s}$, which is the highest speed of the nervous transferring. Also, different structures have different properties for $\delta L / L$ responses, which no longer base on mass calculation since for a larger or small water body, their surface tension almost no shifting.) From here, we also can see, Schrödinger wave function is acquired by fixing " $\pm \delta L_{i} / L_{i}$ " shifting. Binding gravitational waves equal to gravitational waves + surface tension region structures, thus they no longer can separate

\section{Volume 8 Issue 11, November 2019}




\section{International Journal of Science and Research (IJSR) \\ ISSN: 2319-7064}

ResearchGate Impact Factor (2018): 0.28 | SJIF (2018): 7.426

with structures again. Schrödinger wave function wants to avoid structures by fixing " $\pm \delta L_{i} / L_{i}$ " ratios, still unfeasible in bio-systems. Suppose there are 12 surface tension regions there, classical quantum mechanics first believe the " $\pm \delta L_{i} / L_{i}$ " for these 12 surface tension regions is the same, then it believes the resulted energy of the wave function operator to these 12 surface tension regions is randomly on one of the surface tension regions. Such math is only fit for very special conditions, quite challenging to apply in bio-systems. We also need to know, " $\pm \delta L_{i} / L_{i}$ " is eigencycle related; that means even totally the same things will be different if their eigencycle round is different. (In the above discussion, we neglect some minor problems, such as Einstein's field equation takes the mass distribution for calculation; the concept of mass is no longer fit for surface tension region and has to be replaced by $R_{\text {mobility }}$ or $R_{h v}$. Also, "an equation in vacuum" is a classical concept; vacuum $X$ non-vacuum = constant, it is not fully fit for bio-systems. However, these minor problems will not impact on the spinal multi-surface tension ratio discussion.)

Now both Schrödinger wave function and Einstein gravitational wave binding part have become the same thing in CSF multi-surface tension region system; both of them can use the same definition, "regenerative repeatability fluctuation" in bio-multi-surface tension region systems. Definitely, the most important is not the technologically unifying of the above two systems; it is that CSF is the memory of the body. Thinking can happen in the brain; memory does happen in the spinal cord. Now we can understand that the surface tensions at the ventricle surface of the brain are outward 23:1 ratio, and those at the upper sphere of the cerebral cortex are inward 23:1 (Fig.h of Suppl.ExpL.4). The human brain depends on these two leading layers of multi-surface tension region for thinking, transverse from organ tissue to molecular levels of multi-surface tension inversions. (We know the ratio of 23:1 since they have been on the spinal cord. For detailed surface tension distribution dynamics, it is quite challenging to measure directly. Use musical inversion is one of the simple estimation while no more data are available. For gravitational binding, no matter Newtonian or Einstein's theory, once enter into surface tension regions will face big errors since both of them fail to tell why gravity exists; now we can understand the existing of gravity is due to the existing of surface tension region. Bio-systems do more close to this fundamental polarization property of binding gravitational waves than other lower surface tension rate systems we see.)

\section{Surface tension converging superposition and frequency notation}

Albeit Principle of Least Action is so general for surface tension region, no theory in the scientific world to refer to its basis---inversion. Not mention the Newtonian system and Einstein's system, even quantum mechanics as a discipline full of inversion processes, still not refers to it. Superstring theory, which claimed to inclusive all the powerful mathematics for physics, yet fails to refer to it. In contrast, ancient people one thousand years ago already had inversion training. Wudang and Shaolin have developed them into systematic somatic training. For $\frac{d}{d x} \cos (x)=-\sin (x)$, it just means symmetric inversion; in real condition, most of the inversions are asymmetric between two surface tension regions, symmetric inversion means only vertical transfer presents, and asymmetric inversion includes the horizontal transfer of the surface tension. Due to most of the surface tension regions are not evenly distributed, converging training becomes necessary. Converging training just means to make the converge point (the maximum uneven region on a surface tension) of each surface tension region close to each other by means of horizontal \& vertical transferring. Technologically, it includes all the training that strengthens the CSF-ligament conjugations. (Anatomically, synovia directly comes from CSF; serous fluids permeated on the surface or inside of diverse fasciae and tendons are CSF derivatives; there are also a lot of CSF derivatives. CSF correlates ligaments till the cytoskeleton or even to the genomic level. Previously we mentioned that the physical training in (Fig.1j) needs one million repeats to open the spinal joints, which is actually for developing such CSF to cytoskeleton correlations. Such kind of correlations is very weak for untrained people; for correctly trained people, such kind of correlations is very stronger, only depend on strengthened correlations we can open the spinal joints. For older people, their ligaments are quite stiff, that is simply because CSF derivatives can no longer reach diverse subordinate ligaments, or constant $\mathrm{K}$ can no longer tighten enough to issue CSF-ligament conjugations. Whether certain physical training can benefit health is still whether it can strengthen the CSF-ligament conjugation surface tension while training. CSF-ligament conjugation training includes stationary training and fluctuation training (fluctuation training is a little bit similar to modern sports). Due to the lack of effective stationary training, diverse modern sports are not fit for people over a higher performance age.

The only converging training in modern society is the squatting training in body build as in (Fig.2b(1)). Those who difficult to get muscular results can first train this way to converge the constant $\mathrm{K}$ and then take a normal body build training to get good results. (It is not just strengthening the leg muscles, all the muscles beyond squatting still can be further trained after it. However, a warning is that this training must be started at an earlier age; if starting over 28 years old or so, it will not only quite difficult to get the converge effect but also will damage knee joints and lumbar joints.) It is still not a real stationary training; we call it "modern converge training" just because it is the only one that can get some limited CSF training effect as that of an ancient stationary training. (Fig.2b(2)) shows a jade artifact from the Shang dynasty (1250 -1046 BCE) with a kneeling posture. (The device in the waist is an official scepter at that time.) It is the earliest physical stationary training recorded on artifacts, possibly originated from people mimic fetal posture in the womb. In that time, it is not just for personal training; all people in society from the public to the emperor took this posture for all social \& family activities, even horse wagons were designed for this posture. Tatami culture in Japan actually transferred from China in the Tang dynasty (618 to $907 \mathrm{CE}$ ). In China, this social posture was kept till the end of the Qing dynasty (1912 CE), and after that, gradually lost. However, today's Japan still keeps this tradition well than that of its original place---China. (Japanese people averagely the most longevity people in the world are because of this posture and their

\section{Volume 8 Issue 11, November 2019}




\section{International Journal of Science and Research (IJSR) \\ ISSN: 2319-7064}

ResearchGate Impact Factor (2018): 0.28 | SJIF (2018): 7.426

Tatami. China really should recover this tradition; it can make use of daily life for CSF-ligaments strengthening.) It is difficult to find from the Jiahu symbol stage till the Sang dynasty, what is the exact time of this posture began to use and who invented it. We only know it has been the secular basis of physical stationary training for thousands of years until the 1920s. Later, we'll refer to some other advanced converge training; however, those later postures require partial or full religious forbiddance, only this posture is an exception. The significance of the invention and prevalence of this posture in ancient China should not be inferior to the invention of the wheel in history.

Another posture of (Fig.2b(2)) is the basis of all sect physical training. (This posture needs two soles to face heaven, much more challenging than that in (Fig.2b(1)). In Wudang, it generally needs 3 years to gradually upgrade the secular posture into sect posture. "Sect" here not necessarily means religious; any (commercial) groups or even academes with certain inner regulations are also sects; religions are just a small part of sects. The time of posture should be well before the establishment of Shaolin.) The converging function of (Fig.2b(2) (3) is more advanced than that of a squat. In ancient time there are also diverse Wushu (the skills for use diverse ancient weapons) training which same with diverse modern sports required strength and speed, etc., from parts of and not the whole body; also same with modern sports they will not benefit health after certain ages or high-performance stage, people then interweave (Fig.2b(2) (3) with Wushu training to reduce such negative effect. (This is the origin of kungfu, just Wushu or sports mixed with (Fig.2b(2)(3). There are hundreds of recorded sects in history that rely on such level one kungfu training as professions.) We can see that while people are in the postures of (Fig.2b(2)(3), the blood circulation for lower limbs is in stress conditions. CSF with the higher recovering capability will then compensate for the blood circulation of the lower limbs by its strengthened inversion; this in return, can strengthen the stability of CSF-ligament conjugation in that region and finally superpose on the spinal cord in the long term. In modern times, people in kungfu training generally take two hours of such postures daily; in ancient times, it will be much longer than this. For people at an advanced level, they can keep the postures for a few days; means very stronger CSF-ligament conjugation for keeping this surface tension region. (The hematopoietic shifting figure of (Suppl.ExpL.2) also shows the regulation of $\mathrm{CSF}$ for bone marrow hematopoiesis. Only in the bone regions where CSF wave function can reach, the hematopoiesis can be sustaining; if CSF decays in that region by age, the hematopoiesis will equally decaying thus get the patterns in the figure for non-training people. Ancient physical stationary training can greatly expand the hematopoiesis curves in this figure by the postures of (Fig.2b(2)(3).) It is difficult to know the existing time of the posture in (Fig.2b(3)).

The artifact of (Fig.2b(2)) is from the Tomb of Hao Fu. She was the wife of King Ding Wu and also a stronger warrior general, height $1.75 \mathrm{~m}$, and commanded 13000 stronger male soldiers won a lot of battles. The tomb has been dated to 1200 $\mathrm{BCE}$; at that time, Hao $\mathrm{Fu}$ as a woman had already got physical strike training. (Jiahu symbol stage had already belonged to Neolithic times, before that there was long Paleolithic or even earlier period which physical strike had long been used as a punishment all over the world; people use slaps, fists, whips, rods, etc. to beat the wrongdoers in their tribes or prisoners from the enemy, with at least 6 million years of evidenced history. Even today, some of the modern countries still keep physical strike punishment in their law. It is quite curious why only ancient Chinese people could upgrade physical strike punishment into physical training techniques and all over the world failed to do such an upgrade. The time of systematic physical strike training should be some point between the Jiahu symbol stage and the Hao Fu Tome stage or even earlier.) Japanese people as the earlier branch and later nearest neighbors of China have inherited Tatami culture, and ancient Chinese language pronunciations quite successful still failed to inherit physical strike technologies. (In Japanese, there are substantial Chinese characters still kept the ancient pronunciations \& meanings, while in modern Chinese language such things have been evolved for the same characters. The Needham puzzle just wonders why the industrial revolution didn't originate in China, answer in (Suppl. ExpL.10). However, for the puzzle of the unique upgrading of physical strike punishment with millions of years of history into superior healthy technologies, it is really not easy to understand. Even worse, in most western countries, the law bans any physical strike on babies and children. This is contrary to human evolutionary potential.)

Like most of the training, the physical strike will also meet bottleneck after a long period of training. Bottleneck means the CSF-ligament conjugation can't be further strengthened. Shaolin (established in $495 \mathrm{CE}$ ) gradually invented the "Shaolin converge" posture of (Fig.2b(4)) to solve the bottleneck of training. After 10-15 years of diverse physical strike training, people can gradually take this posture by procedure; everyday daily 1-2 hr then mixed with normal strike training, then after 5-8 years can increase the physical strike strength at least 20 times; also diverse other performances are equivalently improved. We can see the equation on the top of the figure; stretch the joint length of the spinal cord will increase all the surface tension regions inside $\mathrm{CSF}$; that is converging. The converging of the spinal cord transfers to the whole body, then all the surface tension regions from the cytoskeleton to organs are also converged. Converge will result in a narrow $\delta K$, thus expand the bottleneck boundary. (Fig.2b(5)) is the "Wudang converging" stationary training for the bottleneck stage, need to sustain the body weight by the back head and the heels (Sanfeng Zhang was born in 1247CE, studied in Shaolin at youth and later establish Wudang sect.). (Fig.2b(6) is a similar method, just take the hanging method. Such training is a stationary training; therefore, must finish $\mathrm{N}=1$ to $\mathrm{N}=6$ training in (Fig.1j(1) and successfully open the spinal joints first as fluctuation training, then can start; generally, start from age 12-18 combined with normal strike training. The ancient book said the highest level of this kungfu could support three persons on his abdomen for over two hours in this posture; however, nowadays only find those can support one person as in (Fig.2b(7)). As we see the bio quantum paths in (Fig.2a), this posture increases the oscillation transferring from $\mathrm{x}, \mathrm{y}$ to $\mathrm{z}$, and also converges the spinal cord since this posture evenly distributes on all 


\section{International Journal of Science and Research (IJSR) \\ ISSN: 2319-7064}

ResearchGate Impact Factor (2018): 0.28 | SJIF (2018): 7.426

vertebrae, the converging effect is much better than that in (Fig.2b(4)). (Use a ruler to measure a people in the posture of (Fig.2b(4)); each cervical vertebra can observably stretch 3-5mm; however, the thoracic and lumbar vertebra not observably shifting to over $1 \mathrm{~mm}$.) Wudang (Taihe) becomes the only level-three ancient physical training is due to this converging posture for spinal training, finalized in 590 years ago (Suppl.ExpL.9). The most important thing for physical training is all the surface tension regions can't be broken. (Attenuation for any surface tension even to limits will not be a problem since the body can recover later. Unprepared people can't perform Wudang and Shaolin converge since broken of surface tension region will happen.) (Fig.2b(8) shows the sampling of CSF in modern times; the blood is just from peripheral tissues and not from CSF. Start from earlier development, CSF has been separated from the blood. (Fig.2b(9)) shows the needles for sampling CSF and a textbook instruction for CSF sampling. These can offer side evidence for the position effect ${ }^{93}$ of CSF. However, we should understand that CSF sampling is a process that will break most of the surface tension in the body, significant lifespan impact. Modern medical sciences know too little about CSF but ignorantly sampling too much from it. (Fig.2b(10) reveals the developmental origin of the spinal cord CSF and derivatives. In summary, kungfu is a physical training system concerning body CSF-ligament conjugations, quite different from diverse other ancient or modern sports that not realize such conjugations. (The strength any person can physically issue doesn't depend on how stronger his muscles are, it depends on the harmonic of CSF-ligament conjugations. Muscles need superposition on the spinal cord to issue physical strengths; stronger muscles first need lower "inner resistance" from the CSF-ligament conjugations to issue efficiently. Also, senility is the weakening of such conjugations. At old age, once the CSF-ligament conjugations are weakening, no matter people have big muscles or small muscles will get the equivalent aftermath. Therefore, the most important is to keep the CSF-ligament conjugations instead of the muscles. Lifespan directly relies on CSF.)

As mentioned before, no theory refers to inversion in the scientific world; only one empirical case of developmental inversion $^{13}$ is reported. The only system which can modulate inversion is actually music harmonic; now we go to this tool. (Fig.2c) shows the fundamental formula of 12-TET that we have mentioned before in quantum superposition, $\boldsymbol{f}_{\boldsymbol{n}}=\boldsymbol{f}_{\mathbf{1}} \times$ $\sqrt[12]{2^{n-1}} ; f_{1}$ has been set as $440 \mathrm{~Hz}$ since 1955 ISO norm. Before that standardization, historically diverse other frequencies $20-30 \mathrm{~Hz}$ up or below $440 \mathrm{~Hz}$ had also been used for centuries. A440 means the No.49 key of a piano is tuned into $440 \mathrm{~Hz}$, then all the frequencies of each key can be calculated by the formula, E.g. the No.50 and No.52 key are $440 \sqrt[12]{2^{1}}$ and $440 \sqrt[12]{2^{2}}$, if the $f_{1}$ is tuned as $432 \mathrm{~Hz}$ then they become $432 \sqrt[12]{2^{1}}$ and $432 \sqrt[12]{2^{2}}$. Not only an 88-key or 108 -key piano is turning in this way but also include all musical instrument. Since there is only one $f_{1}$ we omit it and just write down the Chu's constant $\sqrt[12]{2^{k}}$ in the C major staff notation on the top of the figure. They multiple with $f_{1}$ can get the actual oscillation frequencies of each pitch. The left hand of the figure is a simple harmonic frequency level of a $\mathrm{C}$ major scale. The scales for any kind of musical system are never evenly distributed, as in the figure for a $\mathrm{C}$ major, each note equal to 2 frequency levels, and those between pitch $\mathrm{E}-\mathrm{F}$ and $\mathrm{B}-\mathrm{C}_{\mathrm{octave}}$ are only one frequency level. We call these positions as "converging" regions and marked with red color in the figure. The musical tone just means the position of such converging regions on a scale. (If the converging regions fall on $\mathrm{E}-\mathrm{F}$ and $\mathrm{B}-\mathrm{C}$ of a scale, then it is a major tone; if they shift two levels to D-E and A-B then it becomes a minor. Historically, there still other tones; now only major and minor are still in use. In bio-systems, the regenerative scales and chords could possibly be more complex than these in music; however, the mathematical patterns are the same. For simple, we only use major $\&$ minor triads in the examples of all the converging modulations in this paper.)

(Fig.2d(1)) are some of the most commonly used chords. Music is based on the scale as in (Fig.2d(2), combinations of parts of the scale are chords. (In (Fig.2d(2)), the scale has been transposition just for the convenience of illustration. "Transposition" is routine in music which can be defined by the mathematical operator, $\left|\sqrt[12]{2^{\mathrm{n}-1}}\right\rangle+k=\left|\sqrt[12]{2^{(\mathrm{n}-1)+\mathrm{k}}}\right\rangle$ here $\mathrm{k}, \mathrm{n}$ are integers.) Chords can range from triad till thirteen chords which possess diverse inversions, as in the figure for a triad the root note jump for an octave get the first inversion; the note above the root jump for an octave then get the second inversion, and so on (E.g. C-E-G first inversion $\Rightarrow$ E-G-C $C_{\text {octave }}$, second inversion $\Rightarrow \mathrm{G}-\mathrm{C}_{\text {octave }}-\mathrm{E}_{\text {octave }}$ ). Here any note "jump for an octave" means its oscillation frequency is doubled, as in (Fig.2d(2)), frequency of $\mathrm{C}_{\text {octave }}=2 \mathrm{C}$. (For bio-systems DNA, RNA or cells, etc., inversion takes the same model, if a few genes binding together by a constant $\mathrm{K}$ with certain oscillation frequencies, then one of the frequencies double will get one type of inversions. Senility is simply the weakening of CSF inversion capacity for certain reasons.) Any note has its strengthening \& weakening effect while running in a melody, accompaniment just compensates for the strengthening \& weakening effects or inversion pattern of each note in a melody. Accompaniment of a melody by chords can have different "splicing number", as "columned chords" in (Fig.2d(3) (splicing number =1) or "arpeggio" in (Fig.2d(4)) (splicing number $=\mathrm{k}$ ), here the columned chords and the broken chords are just different in their melody strengthening $\&$ weakening effect binding.) To facilitate the application of Einstein frequency energy and converging superposition in bio-systems, we have transferred conventional staff notation into frequency notation by the manner in (Fig.2e(1)). (Fig.2e(2) shows the scale frequencies used for transferring (omit $\mathrm{f}_{1}$ 440Hz). (Fig.2e(3) and (Fig.2e(4)) are the transferred frequency notations come from staff notations of (Fig.2d(3) and (Fig.2d(4), respectively. This notation system can directly use for playing the piano and composing music, outstanding in children early education for music and math, no need of any accidentals (the non-note symbols in Maj/Min ${ }^{7 \# 9}$ of (Fig.2d(1)); accidentals are used for keeping the same converging points in inversions for staff notation) and people can quickly perceive the composing skills of great musicians like Beethoven without decades of training in a musical school, etc. However, the establishment of this system is not for such musical impacts; it is for real applications in bio-systems for modulating inversion and surface tension region horizontal \& vertical transferring. In music just one $\mathrm{f}_{1}$

\section{Volume 8 Issue 11, November 2019}




\section{International Journal of Science and Research (IJSR) \\ ISSN: 2319-7064}

ResearchGate Impact Factor (2018): 0.28 | SJIF (2018): 7.426

of $440 \mathrm{~Hz}$, in bio-systems there are possibly a few $\mathrm{f}_{1}$ frequencies such as $\mathrm{f}_{1-\mathrm{DNA}(360 \mathrm{bp})}, \mathrm{f}_{1-\mathrm{RNA}}, \mathrm{f}_{1 \text {-stem cells }}$, etc., or even just for a $360 \mathrm{bp}$ segment DNA there is more than one $\mathrm{f}_{1}$; however, no matter how many $f_{1}$ are existing and how complex the system, the structures of biomaterials will not be changed, here the structures just mean the regenerative converging positions will keep the same. (Without this tool, a constant $\mathrm{k}$ binding inversion will be quite challenging to modulate.) From the right hand of (Fig.2f(1), we can see that music converging point represents the frequency energy ideal Le Chatelier's process. From $\mathrm{C}$ to $\mathrm{C}_{\text {octave }}$ the frequency is doubled, and system Le Chatelier's effect will counteract this change, then the frequency energy step for the converging point is reduced to half of a normal step as the ideal result. In "major" tone, the direction of the E-F converging should be reverse to $\mathrm{C}-\mathrm{C}_{\text {octave }}$ shifting direction. Music as a discipline with thousands of years of history is just developed by arranging the Einstein frequency energy ideal Le Chatelier's effect in advanced for composing. Music tonality reveals to us how the ideal frequency energy Le Chatelier's effect means. Bio-systems should be complicated than those of a musical system; however, the concept of ideal frequency energy Le Chatelier's converging is absolutely the same. Now we can see that superposition of music and bio-systems is different from that of quantum mechanics, the latter don't include any Le Chatelier's converge. (Albeit music still calculates frequency steps as $f_{n}=f_{1} \times \sqrt[12]{2^{\mathrm{n}-1}}$, it doesn't directly use these "steps"; they must "regenerative" to give normal notes two steps and converging position for one step as in (Fig.2f(1)). In contrast, quantum mechanics directly uses its calculated energy "steps" from $E \Psi=\widehat{H} \Psi$ or $E=h v$ and no regenerative and converging process; such a direct utilizing is totally different from "regenerative" utilizing. For the application of HiGee in bio-systems, if the rotor takes a uniform speed, then it is the Copenhagen superposition; if the rotor uses some asymmetric pattern like four chambers of heart, then it is converging superposition. We have mentioned the "repeatability fluctuation superposition" definition before; now we can understand that "fluctuation superposition" is actually "converging superposition"; "regenerative" is for integrating environmental signals into superposition converging pattern. The ideal converging superposition is the composition of a music harmonic pattern.)

The left hand of (Fig.2f(1)) reveals the frequency converging positions of each octave are all the same. (Fig.2f(2) shows some of the commonly used chords written into the cyclic format; with this tool, we can see the inversion relations more clearly. In the figure only show one octave, as the first chord $\mathrm{C}_{\text {major }}$ set $\mathrm{C}=\sqrt[12]{2^{0}}$, then in situ chord is $\left(\sqrt[12]{2^{0}}\right)-\left(\sqrt[12]{2^{4}}\right)-$ $\left(\sqrt[12]{2^{7}}\right)$, some people call all these $\sqrt[12]{2^{0}}$ on the root position and other notes shift as in situ, so all the in situ chords should be $\left(\sqrt[12]{2^{0}}\right)-\left(\sqrt[12]{2^{4+12 m}}\right)-\left(\sqrt[12]{2^{7+12 n}}\right) ; 1^{\text {st }}$ inversion will be the in situ root note double the frequency and the second in situ note falls on the root position, $\left(\sqrt[12]{2^{4}}\right)-$ $\left(\sqrt[12]{2^{7+12 \mathrm{j}}}\right)-\sqrt[12]{2^{0+12 \mathrm{k}}} ; 2^{\text {nd }}$ inversion will be the third note on root position, $\left(\sqrt[12]{2^{7}}\right)-\left(\sqrt[12]{2^{0+12 p}}\right)-\left(\sqrt[12]{2^{4+12 q}}\right)$, and so on, here all $\mathrm{m}, \mathrm{n}, \mathrm{j}, \mathrm{k}, \mathrm{p}, \mathrm{q}$ are integers. We omit other outside layers and only show a $\mathrm{C}_{\text {octave }}$. In music, most of the inversions happen in three octaves. However, in bio-systems, the inversion distance (frequency spectrum) could be quite large. Suppose there are three sorts of molecules or cells like the $\mathrm{C}_{\text {major }}$ triads binding by a constant $\mathrm{K}$ with certain frequencies. (Not every molecule can reach ideal frequencies; however, the eigenoscillation part fits 12-TET frequencies well than other parts.) The movement of these molecules, including pass certain membranes or structures still via inversion. From in situ states, the frequency of root molecules or cells doubled and pass through certain membranes or structures, get the $1 \mathrm{st}$ inversion movement, then the frequencies of the second molecules or cells doubled and pass through the membranes or get $2^{\text {nd }}$ inversion movement, and so on. As mentioned before, all the movements of bio-systems running through inversions, food digestion (Suppl. Exp. 15), and even memory are unexceptionally following this model. For a cell or other structures, one direction inversions always accompanied by another direction of inversions; anabolism and catabolism with the same gravitational binding compose inversion relationships.

In music, any composing skills or operations such as chords fluctuation, accompaniment, counterpoint, etc., must under the same tonality, or the converging positions of any levels are the same. So for "major" tone music, all the melodies, accompaniments, notes, etc. are belonging to the "major" tone; for "minor" tone music, all the things are belonging to the "minor" tone. The majority of the piece music only has one tone. Small numbers of piece music have more than one tone, such as chapter one major tone, chapter two shifts to minor tone and chapter three shifts back, etc. However, within one chapter, it is impossible to use more than one tone. Bio-systems still take the same way; all the biomaterials inside one animal can only take one tone at a certain period. Converging pattern is critical for horizontal \& vertical transferring: it must be stable. (From here we can understand what is cancer, as mentioned before, tone just regenerative converging position; some of the bio-materials shift out from correct converge positions are carcinogens. E.g. one type of stem cells have certain inversion relationships with the other two types of cells and can be written as $\mathrm{C}_{\text {major }}$ inversion frequencies as $\sqrt[12]{2^{0}}-\sqrt[12]{2^{4}}-\sqrt[12]{2^{7}}$, diverse inversions could be written as $\sqrt[12]{2^{0+121}}-\sqrt[12]{2^{4+12 m}}-\sqrt[12]{2^{7+12 n}}$ to issue various functions. However, due to certain mistakes, these cells incorrectly become $\mathrm{C}_{\text {minor }}$ inversion frequencies as $\sqrt[12]{2^{0}}-\sqrt[12]{2^{3}}-\sqrt[12]{2^{7}}$, then any types of inversion $\sqrt[12]{2^{0+12 l}}-\sqrt[12]{2^{3+12 m}}-\sqrt[12]{2^{7+12 n}}$ will become tumor cells. Cancerous cells are not just certain cells with certain chemical or biological definitions; they will finally physically include wrong inversion frequencies with other relevant cells, as in the case frequency shifts from $\sqrt[12]{2^{4}}$ to $\sqrt[12]{2^{3}}$ will be tumor cells, the quiddity of which is the shifting of process converging position. The immune system also depends on frequencies or converging positions for judgment; therefore, once immune cells become the same inversion frequency pattern with that of cancerous cells, they will no longer detect and attack the cancerous cells. There are substantial publications claimed Car-T with certain peptidyl or genomic modifications of $\mathrm{T}$ cell then infuse back to "kill" the cancerous cells, etc., now we can understand any interference fails to recover the oscillation strength and also the converging patterns of the immune cells will be impossible to kill the tumor cells, the acquired immune 


\section{International Journal of Science and Research (IJSR) \\ ISSN: 2319-7064}

ResearchGate Impact Factor (2018): 0.28 | SJIF (2018): 7.426

cells not only need correct converging pattern (finally superposed on the spinal cord) but also need enough oscillation strength. All types of cancer originated from incorrect spinal converging superposition.)

(Fig.2f(3) shows the elementary memory model. There are many surface tension regions in bio-systems, and CSF is the highest superposition structure. Memory just the inversion pattern of a few surface tension regions, three surface tension regions on the spinal cord could compose of a memory unit. Complex memory is the composite of such a unit, could be modulated as a small signal activates a large system in the figure. This process includes a lot of rounds of inversions to finally activate the system, the converging position of which still must keep the same. In vivo memory is equivalent to the butterfly effect in complying with the signal converging position. Suppose the butterfly has two types of manner to flap the wings, "major" inversion signal $\left(\sqrt[12]{2^{0}}-\sqrt[12]{2^{4}}-\sqrt[12]{2^{7}}\right)$ or "minor" inversion signal $\left(\sqrt[12]{2^{0}}-\sqrt[12]{2^{3}}-\sqrt[12]{2^{7}}\right)$, then no matter what happens or how complex of the activating system; "major" inversion signal can only activate "major" converge butterfly effect, and "minor" inversion signal can only activate "minor" converge butterfly effect. It is impossible for a "minor" converge signal to activate a "major" converge system under any circumstance. This means any amplification can't change the converging position of the amplified system, or the shifting of converging position in an amplified Le Châtelier's system will greatly decay the amplification level. The strength of the amplification under certain environment strictly depends on the stability of the original converging pattern. In bio-systems, from diverse recovering processes until human memory all observe this principle. We can call this "Basic Law of Evolution". It is originally from the music tonality. The constant $\mathrm{k}$ stability we mentioned before is equivalent to system converging stability. (This is why bodybuilders can perform squatting to enhance the whole-body muscular effects which outside the range of leg muscles and ancient Chinese people developed so many converging training. Modern medical problems such as type II diabetes, atherosclerosis, cirrhosis, organ failure due to AID, and even cancer, etc., are indeed come from converging stability decaying. We just need to find the inversion pattern of them on the spinal cord for modulation.)

(Fig.2g(1)) shows the developmental inversion ${ }^{13}$; from the dorsoventral inversion of the insect body plan, we could know that the development of the spinal cord in vertebrates still needs one major inversion. As in (Fig. 2b(10) CSF originates from the out thin albumin with the highest surface tension; after neural groove folds into the neural tube, these thin albumins still inside the core of the folding; then at a certain stage they need to inversion to the surface of the spinal cord and mix with other sources to form the CSF. This is why we can observe the developmental inversion as in the figure. Yolk sac homing is also with similar surface tension inversion mechanism. (Fig.2g(2) demonstrates the difference between the horizontal and vertical gravitational forces of two objects. For two non-living objects, both the vertical \& horizontal gravitation forces can be calculated by Newtonian equation $F=G \frac{m M}{r^{2}}\left(\mathrm{G}=6.674 \times 10^{-11} \cdot \mathrm{m}^{3} \cdot \mathrm{kg}^{-1} \cdot \mathrm{s}^{-2}\right)$, then the ratio between the horizontal and vertical gravity should be:

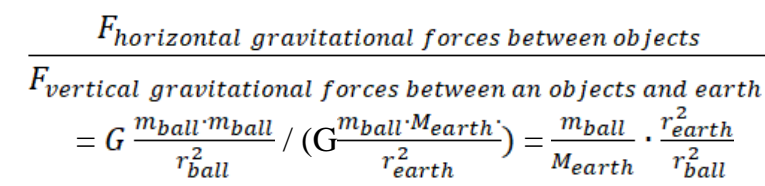

Here $M_{\text {earth }}=5.965 \times 10^{24} \mathrm{~kg}$, Average Radius of the Earth: $6371 \mathrm{~km}$, suppose the metal ball is $1 \mathrm{~kg}$ and the radius is $0.1 \mathrm{~m}$, then the above calculation $=6.80 \times 10^{-11} \approx \mathrm{G}$. This means for all the non-living beings at non-relativistic condition $F_{\text {horizontal }} / F_{\text {vertical }}$ is around $10^{-11}$. That is the range of rigid body Newtonian mechanics. For living beings, the only difference is that the surface tension percentage has been greatly enlarged; the vertical gravitational force still can approximately use the formula $F=G \frac{m M}{r^{2}}$ since bio quantum paths can't change the $\mathrm{z}$ dimensional signals, or the surface tension percentage of a living being will not impact on the surface tension region of the Earth, then the results will not shift too much. For horizontal gravitational force, great surface tension percentages have been integrated into. So the horizontal gravitational force between two living beings is not the Newtonian gravitational force; it is actually the effect of binding Einstein's gravitational waves or surface tension polarized gravity. (If putting a living being and a non-living being on a Cavendish device, we can observe the shifting of horizontal attraction with time since the surface tension region shifting with environmental parameters.) The methods in (Suppl. Exp.6-7) are just indirect and damaging ways for horizontal gravitational forces between living beings; the result is $10^{9}$ enlargements for most of the available multicellular bio-systems at early stages compare to non-living beings. (For human beings, possibly the horizontal gravitational forces between two lovers can attain the upper limit, and for two people dislike each other, the gravitational forces just attain the lower limit; however, still in the $10^{9}$ enlargements range. For single-celled species, we still fail to find the correct way to measure, centrifuge and weight the cell precipitation as in (Suppl. Exp. 7) is not a good method. Single-celled species should be quite lower than $10^{9}$ levels of horizontal gravitational binding. For earlier forms of life, the binding should be even lower than extant single-celled species since the gravitational binding should be a gradually accumulating process corresponding with evolution. Technologies to measure the multi-surface tension or gravitational binding of single-celled species and even viral particles are quite critical to understand earlier evolution. These types of surface tension polarized gravitational forces are quite challenging to be measured by mass concept, HiGee is possibly a choice.) (Fig.2g(3) shows the quadrupole polarization in Einstein's gravitational waves we mentioned before. It does possess horizontal \& vertical transferring as in the figure, just $R_{\text {mobility }}$ or $R_{h v}$ has to replace mass. Horizontal $\&$ vertical transferring is critical for bio-systems. For the frequency notions in (Fig.2e(2),(3), just to show where are the original frequencies come from, we don't make any mathematical simplification. Now, as the highest and lowest frequencies in those two pieces of music are $\sqrt[12]{2^{22}} \& \sqrt[12]{2^{-29}}$, can be simplified into $2 \sqrt[12]{2^{10}} \& 4 \sqrt[12]{2^{-5}}$, all the notes can take this format. The power over 2 is the indicator for the inversion process (inversion number), and the coefficient number before the radical is the indicator for level, or the

\section{Volume 8 Issue 11, November 2019}




\section{International Journal of Science and Research (IJSR) \\ ISSN: 2319-7064}

ResearchGate Impact Factor (2018): 0.28 | SJIF (2018): 7.426

quantum number in quantum mechanics (Quantum mechanics only has vertical, and the horizontal is still the Newtonian system). This way of modulating horizontal \& vertical transferring is much simpler for frequency energy flow. Music takes the simplest way; just use the same note and tone to them can solve the converging problem.

As mentioned before, the memory process happens in the spinal cord, and only thinking happens in the brain. We can understand from (Suppl. Movie 6.0), albeit it is presented as a cell migration model at the start. As in the movie and the figure of (Suppl.ExpL.7), outside detectable signals can be regenerated into three types based on their impact on the cell; "strengthening", "weakening", and "fluctuation". (Any signal enters into the cell membrane still takes the model of (Suppl. Movie 8.3), the white barrier is cell membrane, another barrier in the environment close to it. Signals oscillation between the environmental barrier and the cell membrane, part of them enter into the cell membrane by inversion, inside the cell still via relay inversion to reach the inner surface tension layer. (Suppl. Movie 6.0) omits all the intermediate inversion processes, just show signals direct impact on the inner eigen surface tension layer.) Three sorts of signals, especially the fluctuation types, are all "regenerative" signals. Suppose a ring of bacterial inner eigen surface tension region or genome active part can "remember" 6 fluctuation signals in a certain period, and the cell has already growth with 3 fluctuation signals in that period, then while the $4^{\text {th }}$ fluctuation signal add into, it will be compromised into an eigensignal which composed of these 4 fluctuation signals; while the $5^{\text {th }}$ fluctuation signal enters into the system, even everything of $4^{\text {th }}$ and the $5^{\text {th }}$ signals are totally same at every aspect, the results are different: one condition is an eigensignal establish from 4 signals, another condition is an eigensignal establish from 5 signals, "regenerative" comes from here and just mean based on all previously available signals to establish the eigensignal, not the Copenhagen superposition. All the "regenerative" signals have "converging positions", and the superposition is converging superposition. While evolution hits advanced species like human beings, the condition is the same, just all detectable signals superposed on the spinal cord and establish eigensignal for a certain period. For a newborn baby the spinal cord even can't support the head, diverse signals from eating, eyes, ears, noses, touch etc., gradually superposed on the spinal cord and establish the converging patterns (what frequency notation represents just the ideal pattern instead of all patterns), the shifting of converging pattern of memory need long term training, estimated 3-5 years from the baby stage and with the increasing of age the shifting becomes increasingly difficult. For the somatic body, the time for shifting converging patterns will be longer, at least double to 6-10 years at an early age. (Shaolin converge, or Wudang converge just ancient physical training for recovery of original spinal converging patterns which have been driven to divergent by ordinary life. It generally needs decades. Even for a simple bacterium, the shifting of the converging pattern is quite rare; it is estimated that no more than three converging shiftings can be tolerated in one lifespan.) So for a cell or a human being, the "memory" just bundles of regenerative fluctuation signals superposed on the genome or the spinal cord with one or bundles of eigensignals, as we show in the movie and the figure. While "thinking", it needs to take the original signal interacting with the eigensignal. The simplest memory just one fluctuation signal participates in bundles of signals on the superposing structure to establish an eigensignal, and the simplest thinking just this signal interacting with the eigensignals and come out for using, albeit the complex memory and thinking processes are entangled together. We can simply regard memory as frequency increase inversions and thinking as frequency decrease inversions with different ration in diverse processes. (Brain neuron CNV show wide variability (9 to $100 \%$ of brain neurons in different studies) ${ }^{33}$ can offer side evidence.) For the integration of the converging pattern of the spiritual world and somatic body, refer to (Suppl. ExpL. 10), this is the driving force of evolution.

From the simple model of thinking, we also can understand the reason that AI lacks creativity as that of human beings. When humans need to solve a real problem, it has to unexceptionally composed of two parts: "observe the rules" and "shift the rules" with a different ratio as the formula: constant $=$ (observe the rules) $\times($ shift the rules $)$. Such conjugation comes from the converging superposition, albeit no one consciously realizes this equation in one's solutions. No matter how deep this formula is hidden in human solution, none item can be zero. AI is running by the probabilities under present rules; therefore, the strength of model-able AI or "deep-learning" is "observe the rules", the weakness of which is impossible to find any "shift the rules" solution without extra outside system input. It is then groundless for AI to get above human equation. Practically we can see what $\mathrm{AlphaGo}^{94}$ learned from humans are the skills to play go "under the rules", not the skills to "shift the rules" such as the invention of the game or apply the rules from the go game to other fields. For these reasons, it is still a tool for human beings no matter how powerful it is performing in a fixed rule condition. Some people attributed the malfunction of AI in multi-task jobs as lack "common sense" and still didn't clear what exactly the "common sense" is. The human equation seems too "clumsy" at first; however, the advantage is while needing to "shift the rules", any info available with the same converging patterns can be used as "common senses". It is actually not the so-called "common sense" that turns the tide; it is "equal converging pattern" info that offers the solution. (Human is an open system; any info with the same converging patterns can go into the brain for help. AI is an isolated system; no way to acquire shifting information unless de novo re-modeling by humans. Not mention great shifting of rules; tiny shifting such as board from $19 \times 19$ to $18 \times 18$, etc., must $d e$ novo re-modeling by humans. Suppose we arrange AI play the go game with a human player from $9 \times 9$ to $10009 \times 10009$, a totally 10000 types of board size. There is no need for any re-modeling of the human player; the human brain can use "same converging" info automatically. In contrast, AI does need 10000 times of de novo re-modeling, and each re-modeling must be operated by humans since the self-remodeling capability of AI is zero. (de novo re-modeling just means what AI "deep learned" from $19 \times 19$ board is useless for $18 \times 18$ board application.) Not only the remodeling needs human but also the human re-modeling does need large enough data. Right now AI only defeat human in a $19 \times 19$ board with huge human competition records for deep

\section{Volume 8 Issue 11, November 2019}




\section{International Journal of Science and Research (IJSR) \\ ISSN: 2319-7064}

ResearchGate Impact Factor (2018): 0.28 | SJIF (2018): 7.426

learning; if AI needs to compete with a human player in above 10000 types of board size, it even lacks enough data to re-modeling other 9999 board sizes. AI can't transfer what deep learned from $19 \times 19$ board to other board sizes and compete with the human. Le Châtelier's effect of human thinking offers re-modeling capability for defeating AI under such a small shifting rule.) From this case, we could know if we wish to solve the malfunction of AI, the method is still to try the above human equation for AI running since only rely on human de novo re-modeling can't solve all the problems. However, even in the future, people can really solve the problem of "self re-modeling" capability for AI; "available resources" are still restriction factors. As the story of AI war, the side with AI only has limited munitions and inferior generation of weapons, they still can't match another side with ample supply of superweapons, like AI can't help one group of people with bows and arrows to declare war with another group of people with machine guns; such restriction factors are called "available resources". Also, AI still faces the challenging of model-ability. We can say that human-guided model-able AI can defeat another group of people without AI tools under "equal rules" and "equal available resources" conditions; however, AI can't govern the human world without human resources. Legendary "AI governs human" story can only be possible under the guidance of another group of humans; what happened is still a "human governs human" quiddity instead of an "AI governs human" story.

All our previous discussion can be generalized into the Basic Law of Evolution as:

Life on Earth is a multi-surface tension region gravitational polarizing Le Chatelier's regenerative system, the least Einstein's frequency energy for each surface tension region is structure-correlated inversion energy and the structural stability relies on its inversion stability. Any inversion capacity depends on an attached network will keep a relatively stable converging pattem for the horizontal \& vertical transferring of this network until it is regenerative.

We can understand that the law only deals with surface tension parameters, a little bit different from Newtonian physical parameters described in his three laws of motion that only work while surface tension can be neglected. E.g., the third law says $F=-F$, most of the Newtonian objects we see do balance in this way. However, surface tension is an exception, all the motion units inside it must be modulated by bio quantum paths with different $\mathrm{z}$ dimensional oscillation ratios; all these ratios can't be 1:1 since 1:1 means the surface tension region is in the weakest state and close to dispersing. The first law says that every object in a state of uniform motion tends to remain in that state of motion unless an external force is applied to it. This law means the less an object gets impact, the more it can keep uniform motion. However, for the molecules or large structures in surface tension region, they must depend on the resonation and inversion from others to survive. Once they don't get any impact from other molecules, their motions will be stopped; molecular motion decaying means the surface tension region will disperse, or in life means the molecules or structures will be death from the system. The state that less impact from others, the more can remain its Newtonian inertia state only fit for objects which surface tension can be neglected, will never adapt to surface tension parameters. The second law is the calculation of diverse vector forces based on the first law. However, a vector force is generally no impact on a bio quantum path unless it can shift all the $|z+| /|z-|$ ratios in the whole surface tension, not just shift one bio quantum path as it superimposes with a vector force. A vector force itself has no storage function for other vectors; a bio quantum path does possess storage function for other bio quantum paths if the sustaining signal is continuously available. Such an "innate" storage function is the actual reason for eigencycle. Most important, the stationary reference in the surface tension region will be an anti-unitary structure or on the converging parts of the multi-surface tension regions. This is totally different from the stationary reference of a Newtonian system. The latter just means a reference we can use to measure; in bio-systems, the more stationary of a structure, means the more inversions of it compare to other correlated structures to the whole system. (An antibody (Ab) or an immune -globulin (Ig), is a Y-shaped protein produced mainly by plasma cells for the immune system to identify invaders, presenting antigen, and issuing the attack. As a discipline, immunology never explains the exact mechanism of such an acquired immunity. The reason is due to all the bio-structures have a characteristic bio quantum path $|z+| /|z-|$ ratio; once detect this ratio, the immune system will make antibodies and all the available resources to crush this characteristic $|\mathrm{z}+| /|\mathrm{z}-|$ ratio to $1: 1$ to damage unwelcome agents. Any bio quantum path with $|z+| /|z-|$ in $1: 1$ ratio will get the less resonation from others, then it will in the weakest state. The primitive immune system, like CRISP/CAS 9 is still functioning by the inversion between a sequence and the palindromes. This is the mechanism of immune reaction. For this reason, it not only deals with invaders, any own cells with wrong $|z+| /|z-|$ ratio with system requirement will equally get immune clearance.) We also note, mass is not a surface tension parameter since it has to exist under the condition that the internal relative mobility of an object are neglect-able, once this condition is moved even inertia mass is not in proportion to gravitational mass. The third law is based on the mass concept, and the $\mathrm{z}$ dimensional oscillation ratio of the bio quantum path is based on the driving stability inside the surface tension region.

There are many ways for validating the model; we even have a clinical trial (Suppl. Exp. 16); however, the best one is still spaceflight. On Earth, a simple bone fracture can recover if it gets alignment and fixed. If an animal is in a spaceflight short of gravity, the bone fracture will be notoriously difficult to recover. Also, the risk of atherosclerosis will greatly be enlarged. Possibly just a few months in such an environment will equivalent to decades on Earth to accumulate such problems. Osteoporosis is also such a time quickening process. For an animal in a short term spaceflight, the decrease of elastic of blood vessels has been observed quickly; the long term will then be atherosclerosis. With similar mechanisms, animals or humans in spaceflight will face severe ED problems at once due to lower gravity. And animal growth, especially the spinal column, will be severely reversed in spaceflight in the long term. Empirically, the resistance of physical strike (empirical bio-inertia, can be calibrated by certain mass strikes on a certain part of the body with a certain speed, $p=m v$ ) will be greatly reduced for the same animal

\section{Volume 8 Issue 11, November 2019}




\section{International Journal of Science and Research (IJSR) \\ ISSN: 2319-7064}

ResearchGate Impact Factor (2018): 0.28 | SJIF (2018): 7.426

or human, no matter these animals or human beings have gotten physical training before. All these are easy to be predicted, just not find relevant literature. However, other changes, such as muscular hypotrophy, protein express change and dysfunction of the vascular endothelium, etc., have been published ${ }^{95}$. Following the model, the story that takes Mars as the next habitat of human beings is not as simple as people thought it would be. The gravity of the Mars only $38 \%$ with that of Earth, even if we can find water and bring some species on Earth to Mars to strengthen our inner constant $\mathrm{K}$, we are not evolved from there with billions of years of gravitational binding,; therefore, finally either mankind fail to adapt to that planet or evolve to a species which totally different from those of us on Earth. It's just a seeding place instead of a habitat. We still need to take care of the environment \& civilization of our planet instead of migration to a new place that short of gravity.

\section{Life abiogenesis in a whirlpool by surface tension inversion and membrane folding}

As mentioned before, life originated on Earth around 1 billion years after the formation of Earth [1, 2]. For this geological period, short of fossil or geochemical evidence for earlier life has left ample scope for hypotheses, which fall into two main groups: Earth origin and panspermia [96]. Panspermia just due to people lacks the understanding of surface tension and gravitational binding quiddity of life. For Earth origin, the theory of RNA world [97] and the iron-sulfur world [98] has suggested. Such chemical origins lack physical consideration that is actually steering evolution. Life evolution should geologically start from Hedean [99], and the primary biomolecules should from "Miller concoctions". Miller [12] experimented in 1953 with a continuous spark discharge at 60,000 Volts through a flask containing the gases identified by Urey, along with water. The aqueous solution showed 25 amino acids (the main ones being glycine, alanine, and aspartic acid), several fatty acids, hydroxyl acids, and amide products. Following his idea, diverse modification experiments have also carried out by many people. E.g. ribose and deoxyribose were acquired in 1966 by dealing the $\mathrm{HCHO}$ with uv and $\gamma$-ray. Cytosine acquired by heating $\mathrm{N} \equiv \mathrm{C}-\mathrm{C} \equiv \mathrm{CH}$, $\mathrm{KCN}$, and water under $100^{\circ} \mathrm{C}$ (1966). Adenosine can be by UV dealing with a diluted solution of adenine, ribose, ethylmetaphosphate, and phosphate (1977), etc. Even a long fatty acid chain can be acquired by UV dealing the $\mathrm{CO}_{2}$ and $\mathrm{CH}=\mathrm{CH}$, most of the small molecules acquired by simulating certain primordial Earth conditions. All these could be called "Miller concoctions". The surface tension region of these earlier organic small molecules is still non-living beings; only one surface tension region. Molecules or molecular agglomeration inversion is a very general phenomenon. All the physical and chemical reactions are based on inversions. Walden inversion ${ }^{100}$ was first observed by chemist Paul Walden in 1896. He was able to convert one enantiomer of a chemical compound into the other enantiomer and back again to establish a Walden cycle as in (Fig.2h (1) ): (+) chlorosuccinic acid (1 in scheme 1$)$ was converted to $(+)$ malic acid 2 by action of silver oxide in water with retention of configuration, in the next step the hydroxyl group was replaced by chlorine to the other isomer of chlorosuccinic acid
3 by reaction with phosphorus pentachloride, a second reaction with silver oxide yielded (-) malic acid 4 and finally a second reaction with $\mathrm{PCl}_{5}$ returned the cycle to its starting point. Most molecules in the natural environment, even as simple as a water molecule, can form two enantiomers around a chiral center in responding to environmental signals. (Fig.2h (2)) shows a water molecular complete nuclear permutation inversion (CNPI), this type of inversion even concerns with the nuclei. (In (Fig.g) of (Suppl. ExpL. 4) we have a cell bi-membrane model, which is utilizing surface tension inversion to correlate outside bi-layer and inner bi-layer. Now we can see that the bi-membrane model has already incorporated inside a water molecular CNPI. However, molecular inversion is rarely reported again, possibly because people don't believe the significance of it in the mainstream study. The molecular inversion in the non-living system is random; evolution just resonates these random molecular inversions into surface tension region, and further superposed them into multi-surface tension regions. Inside surface tension regions, individual molecular inversion becomes a system inversion which incorporated bundles of other molecules (From here, we also can understand the weakness of classical quantum mechanics, the math of this discipline shutoff constant $\mathrm{k}$ binding and inversion; therefore, the process of particle spin from one direction inversed to another direction is challenging to bind with other processes. In bio-systems, an inversion even in the molecular level possibly binds to diverse other processes and potentially can issue a butterfly effect under certain conditions. Classical quantum mechanics then shuts itself for modulating these processes. Today, most of the physical parameters are still Newtonian physical parameters; even subsequent theories don't change this condition. The physical parameters of quantum mechanics should be surface tension physical parameters; just due to some of the reasons, it fails to realize this point and become an incomplete discipline. The difference between Newtonian parameters and surface tension parameters is modulated by the "operator" manner or not. We even can define a quantum into a bio quantum path; wave-particle duality is then clearer. Most people don't know what exactly a quantum is and use the classical formula $E=$ $n h v$ to explain it. Albeit this way can justify "quantum superposition" into a unitary mathematical calculation, it easily creates errors since it fails to reveal why quantum exists. If we regard $h v$ as the "least fluctuation unit" and define a "quantum" into a bio quantum path, then not only actual measurement and mathematical inversion calculation becomes feasible, but also we can clearly see where a "quantum" is born; it just born from a surface tension region-the born of such a "quantum" also ecbolic the challenging of thermodynamics. An isolated system is only an ideal model that is non-existing in the real world. No matter which kind of technology we used to create an isolated system, it just means the energy difference between the inner and outer sides of the boundary. This difference can be modulated by the bio quantum path layer with different $\mathrm{z}$ dimensional ratios; these ratios will be inversion at certain conditions in the long term. The second law of thermodynamics is then a fragile model to survive while any inversion happens.)

(Fig.2h (3) (4) (5)) modulate the DNA, cell membrane, and prokaryotic cell membrane folding structures by bio quantum 


\section{International Journal of Science and Research (IJSR) \\ ISSN: 2319-7064}

ResearchGate Impact Factor (2018): 0.28 | SJIF (2018): 7.426

path inversion models. The common basis of them is the phosphate, an ingredient of the soupy bubbles play by children. Now the critical step in evolution is how these bubbles can naturally get bi-layer by inversion and then memorizes some molecular inversion patterns and transfer to other molecules or molecular agglomerations. On Earth's environment, the only way to do so for the primordial molecules should be happening in the whirlpool.

The left hand of (Fig.2i) shows a whirlpool, it roughly has three areas; on the top the thickness of the surface tension region is not evenly distributed; however, the net surface tension forces are inward to water body with various oscillation strengths (the inversion molecular agglomerations become evaporation thus the percentage of inversion is too lower), so we call this part "surface tension inward area". The middle of the whirlpool is the "surface tension stronger inversion area", in here, all the things inside water, no matter dissolve or not, will experience very vigorous inversion. We call the bottom part of the whirlpool as the "inversionstable-bubble area" since only bubbles are essential "products" for evolution. Bubbles pass through the whirlpool for one round will experience a lot of inversions; the same with the in vivo conditions, the number of inversions will contribute to the stability of the bubble. Most bubbles are broken before they back to the surface or to other whirlpools; after back again, the same surface inversion bubbles will relatively stable and contribute to the stability of the bubbles on the surface. All the reverse surface tension bubbles are quite ephemeral; they need to fuse with a normal surface tension bubbles, and the majority of the fusions only result in normal surface tension bubbles, only very little chance can form a bilayer with both sides are hydrated and inner dehydrated tail in the middle. The thickness of one lipid layer includes $0.7-1.0 \mathrm{~nm}$ fully hydrated head, 1.2-1.8nm fully dehydrated tail, and $0.3 \mathrm{~nm}$ intermediate region. Generally, the diameter of most prokaryotic cells is around 1um (rod or spiral-shaped cell roughly equal to coccus cell in diameter). Suppose a lum bubble has 1 bilayer outside, then we can calculate the surface tension between the outside layer and the inner layer of the bilayer. $V=\frac{4}{3} \pi r^{3}, \frac{V_{\text {outer layer }}}{V_{\text {inner layer }}}=\frac{r_{\text {outer }}^{3}}{r_{\text {inner }}^{3}}$, if $r_{\text {outer }}=r_{\text {inner }}$ it will difficult to establish surface tension region. So it should the out layer takes the upper limit, and the inner layer takes the lower limit of the length of lipids, the intermediate region should be added to the outer layer. We then take outer layer thickness as $3.1 \mathrm{~nm}$ and inner layer thickness as $1.9 \mathrm{~nm}$, use our formula $\frac{r_{\text {outer }}^{3}}{r_{\text {inner }}^{3}}=\frac{3.1^{3}}{1.9^{3}} \approx 4: 1$. So we could know the polarity of the bilayer is around 4:1, if we use bio quantum paths to express these earlier bi-layers, the ratio of $\mathrm{z}+/ \mathrm{z}$ - is around $4: 1$, the gravitational binding within surface tension region is also 4 times as that of before binding. (Such a polarity is only for earlier established bilayer without life; with evolution and diverse constant $\mathrm{k}$ binding, this ratio will shift greatly with the integrating of new structures. DNA should have similar polarity as in the figure, and palindromes should be the stable state of surface tension region; coded gene and the gene expression will shift the ratio widely. The reason why PCR can copy DNA segments still because of inversion; the temperature cycle continuously unwinds (helicase) and anneals the DNA; as in the (Fig. 2h (3)), DNA double-strand unwinding means inward surface tension inversion to opposite direction, and annealing inversion back, such a standard inversion process drives the PCR. The actual $\mathrm{z}+/ \mathrm{z}$ - ratio in the in vitro process should be diverse.)

Get bilayer is only the first step, then need to integrate various primordial molecules into the surface tension region by inversion processes. As our bio quantum path modulation in (Fig.2h(3), DNA should first form in the middle of the bilayer and later fold inside the bubbles by geological time. The processes are still by whirlpool inversions. Other similar size molecules such as peptide still take the same way, first establish inside the bubble bilayer then fold in the bubble later. These processes are quite difficult to simulate by a laboratory whirlpool since they need geological time; however, for a short DNA segment, a short peptide, or similar size molecules, etc., can be absorbed into the middle of an artificial bubble in the lab, and the respiratory chains of extant cells are also inside the unit membrane. Moreover, in all vertebrates, CSF is unexceptionally developed from thin albumin (Fig.2b (10); these hard facts strongly prove that $\mathrm{DNA}(\mathrm{s})$ are first formed inside the bubble bilayer and later fold into the bubble. For the bi-membrane model in the (Fig.g) of (Suppl.ExpL.4), it should be formed by folding. (Fig.2h(5)) shows a prokaryotic cell membrane folding structure. Chemosynthesis bacteria (Nitrifying bacteria, sulfur- oxidizing gamma and epsilon proteobacteria, the Aquificae, methanogenic archaea, and the neutrophilic iron-oxidizing bacteria, etc.) and photoauto -trophic bacteria (purple sulfur bacteria, cyanobacteria) generally have such membrane folding structures, albeit the existing of mesosome is controversial (such debate is due to some people so trust Cryo-EM can fully snapshot bio-structures, the belief is actually doubtable). All these earlier bacteria possess the capability of respiratory chain cell membrane on-site inversion to acquiring reducing power (NADH or NADPH) from ATP (Heterotrophs transfer electrons from NADH to receptors to get ATPs; the autotrophs can cost ATP and reversely raise electrons from receptors to synthesis $\mathrm{NADH}$, we call this as respiratory chain inversion; receptor in advanced species is only $\mathrm{O}_{2}$, in bacteria also include other inorganic matters, such as $\mathrm{NO}_{3}^{-}, \mathrm{SO}_{4}^{2-}$, $\mathrm{Fe}^{3+}{ }^{3+} \mathrm{CO}_{2}$, and organic matters, etc.; all respiratory chain steps, such as $\mathrm{NADH}+\mathrm{H}^{+} \rightarrow \mathrm{FMN} \rightarrow \mathrm{CoQ} \rightarrow$ cytb $\rightarrow$ cytc $\rightarrow$ cyta $\rightarrow$ receptor, are exceptionally follow the bio quantum path inversion model we use to explain Einstein's photoelectric effect. That is, from NADH complex inverses to FMN complex, FMN complex inverses to CoQ complex, and so on; each step is a surface tension region; in this respiratory chain, it is seven surface tension regions within the cell membrane. Besides "relay" inversions on the textbooks, harmonic inversion, such as from NADH to $\mathrm{CoQ}$, or cytc, etc., could also happen under certain conditions.); bioluminescence that is so general for ocean species is a similar type of partial inversion; even ATPase is an inversion structure on cytoplasm membrane. From the cell membrane folding, these species can get the reverse surface tension membrane inside the cell, and this process is the earlier basis of acquiring multi-surface tension regions in evolution. Gravitational binding plays a critical role in the horizontal \& vertical transferring of multi-surface tension region systems. It can be modulated by Gauss's divergence theorem (Suppl. ExpL. 11). The bottom

\section{Volume 8 Issue 11, November 2019}




\section{International Journal of Science and Research (IJSR) \\ ISSN: 2319-7064 \\ ResearchGate Impact Factor (2018): 0.28 | SJIF (2018): 7.426}

of (Fig.2h(4)) is a capillary tube modulated by bio quantum paths, the black arrow is the horizontal transferring, and the $\mathrm{z}$ dimension of the bio quantum paths on the tube wall is the vertical transferring directions. In a capillary tube, only while the size of the tube close to liquid surface tension region thickness, we can then get this horizontal transferring to raise the height of the liquid surface. If we change the diameter of a capillary tube into that of an animal intestine, the driving force for the feces in the intestine is still this type of horizontal direction, as in the figure. Now the size of the intestine has been enlarged greatly than a capillary tube $(0.2 \mathrm{~mm}$ diameter for pure water enlarged to few centimeter gut size); in such an enlarged size, the intestine still can issue such horizontal surface tension forces to drive the feces; that fully depends on the gravitational polarization (Molecules or molecular agglomerations are vertically inversing to the gut wall; all asymmetrical inversions possess vertical transferring. These vertical transferring pile together, then drive the fecal movements.). For estimation of the earlier evolution, from the molecular level single surface tension region of primordial molecules evolved into human takes around 35 billions of years and the gravitational binding is enlarged to $10^{9}$, averagely just 3.5 years can get ten folders of gravitational binding for bio-systems. Estimated viruses have $10^{3}$ gravitational bindings, then just a few thousands of years the life forms similar to viruses could come out (they can reside on higher surface tension flow and not necessarily with a host). If bacteria have $10^{6}$ of gravitational binding, then just 0.1 million years could come out. Possibly such kind of "average" calculation method still needs further modifications since earlier life developed very slowly, and advanced species developed in the acceleration. Also, we still lack methods to measure the surface tension or gravitational binding of viruses and single-celled species; their empirical data will be quite critical to understand the time frame of earlier evolution.

\section{Ethnic Statement}

The animals used in this study are approved by Tsinghua University Animal facility with (IACUC) approbation. The human umbilical cord blood sample had gotten the written consent of the donator. NKT clinical trial complies with regulations.

\section{Acknowledgments}

Thanks to Dr. Minghui Zhang of Medical Center, B343 for designing and organizing NKT cell cancerous metastasis inhibition clinical trial; Dr. Geng Tian, E226, Dr. Jing Liu, B201, Dr. Anming Meng, C121, and Dr. Yanan Du, C232 for lab and material support. Thanks also to decades of ancient physical training from Ke Ping Fan, Guang Cheng Liu, and Gong Han Lu.

\section{Authors' contributions}

Lai,Y. received decades of ancient physical training, perceived the models, performed all the experiments, and wrote the manuscript.

\section{Competing financial interests}

The authors declare no competing financial interests.

\section{Supplementary information}

Supplementary information: www.innoen.org

\section{References}

[1] Manhesa, G., Allègre, C.J., Dupréa, B., Hamelin, B. Lead isotope study of basic-ultrabasic layered complexes: Speculations about the age of the earth and primitive mantle characteristics. Earth and Planetary Science Letters, 47(3), 370-382(1980).

[2] Schopf, J. W., Kudryavtsev, A.B., Czaja, A.D., Tripathi, A.B. Evidence of Archean life: Stromatolites and microfossils. Precambrian Research, 158, 141-155(2007).

[3] Ohtomo, Y., Kakegawa, T., Ishida, A., Nagase, T., Rosing, M.T Evidence for biogenic graphite in early Archaean Isua metasedimentary rocks. Nature Geoscience, 7, 25-28(2014).

[4] Noffke, N., Christian, D., Wacey, D., Hazen, R.M. Microbially Induced Sedimentary Structures Recording an Ancient Ecosystem in the ca. 3.48 Billion-Year-Old Dresser Formation, Pilbara, Western Australia. Astrobiology, 13, 1103-1124(2013).

[5] Novacek, M. J. "Prehistory's Brilliant Future". The New York Times. ISSN 0362-4331. Retrieved 2014-12-25.

[6] Mora, C., Tittensor, D.P., Adl, S., Simpson, A.G.B., Worm, B. How Many Species Are There on Earth and in the Ocean? PLOS Biology, 9(8), e1001127 (2011)

[7] Darwin, Charles (1859). On the Origin of Species by Means of Natural Selection, or the Preservation of Favoured Races in the Struggle for Life (1st ed.). London: John Murray. (The book is available from The Complete Work of Charles Darwin Online. Retrieved 2014-11-21.)

[8] Lewontin, R. C. The Units of Selection. Annual Review of Ecology and Systematics. 1, 1-18 (1970).

[9] Kimura, M. The neutral theory of molecular evolution: a review of recent evidence. The Japanese Journal of Human Genetics, 66, 367-386(1991).

[10] Rechavi, O., Minevich, G., Hobert, O. Transgenerational inheritance of an acquired small RNA-based antiviral response in C.elegans. Cell. 147, 1248-1256(2011).

[11] Brack, A. (1998). Introduction. In André Brack. The Molecular Origins of Life. Cambridge University Press. p. 1. ISBN 978-0-521-56475-5. Retrieved 2009-01-07. "Aristotle gathered the different claims into a real theory.

[12] Miller, S.L. A Production of Amino Acids Under Possible Primitive Earth Conditions. Science 117, 528-529(1953).

[13] De Robertis, E.M., Sasai, Y. A common plan for dorsoventral patterning in Bilateria. Nature. 380, 37-40 (1996).

[14] Grøn, Ø., Hervik, S. Einstein's General Theory of Relativity: With Modern Applications in Cosmology. Springer Science \& Business Media. p.180 (2007). ISBN 978-0-387-69200-5.

[15] Einstein, A. The Foundation of the General Theory of Relativity. Annalen der Physik. 354, 769-822 (1916).

[16] Adler, R., Bazin, M., Schiffer, M. Introduction to General Relativity (2nd ed.). New York: McGraw-Hill. p345 (1975). ISBN 978-0-07-000423-8

[17] Elgar, G., Vavouri, T. Tuning in to the signals: noncoding sequence conservation in vertebrate genomes. Trends Genet.24, 344-52(2008).

[18] Pingoud, A., Jeltsch, A. Structure and function of type II restriction endonucleases. Nucleic Acids Research. 29, 3705-27(2001).

[19] Cain, D., Erlwein, O. Grigg, A., Russell, R.A., Mcclure, M.O. Palindromic Sequence Plays a Critical Role in Human Foamy Virus Dimerization. Journal of Virology 75, 3731-3739(2001)

[20] Willwand, K., Mumtsidu, E., Kuntz-Simon, G., Rommelaere, J. Initiation of DNA Replication at Palindromic Telomeres Is Mediated by a Duplex-to-Hairpin Transition Induced by the Minute Virus of Mice Nonstructural Protein NS1. The journal of Biological Chemistry 273, 1165-1174(1998).

[21] Arbely, E., Khattari, Z., Brotons, G., Akkawi, M., Salditt, T., Arkin, I.T. A Highly Unusual Palindromic Transmembrane Helical Hairpin Formed by SARS Coronavirus E Protein. J. Mol. Biol. 341, 769-779(2004).

[22] Bohenzky, R.A., Lefebvre, R.B., Berns, K.I. Sequence and symmetry requirements within the internal palindromic sequences of the adeno-associated virus terminal repeat. Virology, 166, 316-327 (1988). 


\section{International Journal of Science and Research (IJSR) \\ ISSN: 2319-7064 \\ ResearchGate Impact Factor (2018): 0.28 | SJIF (2018): 7.426}

[23] Wu, X., Li, Y., Crise, B., Burgess, S.M., and Munroe, D.J. Weak palindromic consensus sequences are a common feature found at the integration target sites of many retroviruses. Journal of virology, $\mathbf{7 9}$, 5211-5214(2005)

[24] Minot, S., Bryson, A., Chehoud, C., Wu, G. D., Lewis, J. D., Bushman. F. D. Rapid evolution of the human gut virome. PNAS, 110, 12450(2013)

[25] Mccarroll, S. A., Altshuler, D. M. Copy-number variation and association studies of human diseases. Nature Genetics. 39, 37-42(2007).

[26] Taniguchi, Y. et al. Quantifying E. coli Proteome and Transcriptome with Single-Molecule Sensitivity in Single Cells. Science. 329, 533-538 (2010)

[27] Koning, A. P. J. D., Gu, W., Castoe, T. A., Bazter, M. A., Pollock, D. D. Repetitive elements may comprise over two-thirds of the Human genome. PLOS Genetics. 7 (12), e1002384. (2011)

[28] Zarrei, M., Macdonald, J. R., Merico, D., Scherer, S. W. A copy number variation map of the human genome. Nature Reviews Genetics. 16, 172-183(2015).

[29] Sharp, A. J. et al. Segmental Duplications and Copy-Number Variation in the Human Genome. The American Journal of Human Genetics. 77, 78-88(2005).

[30] Macdonald, M. et al. A novel gene containing a trinucleotide repeat that is expanded and unstable on Huntington's disease chromosomes. Cell. 72, 971-983(1993).

[31] Perry, G. H.et al. Diet and the evolution of human amylase gene copy number variation. Nature Genetics. 39, 1256-1260(2007).

[32] Korbel, J. O. et al. The current excitement about copy-number variation: how it relates to gene duplications and protein families. Current Opinion in Structural Biology. 18, 366-374(2008).

[33] Rohrback, S., Siddoway, B., Liu, C.S., Chun, J. Genomic mosaicism in the developing and adult brain. Dev Neurobiol. 78, 1026-1048(2018).

[34] Yang, Y., Topol, L., Lee, H.,Wu, J. Wnt5a and Wnt5b exhibit distinct activities in coordinating chondrocyte proliferation and differentiation Development 130,1003-1015(2003).

[35] Shahriyari, L. A new hypothesis: some metastases are the result of inflammatory processes by adapted cells, especially adapted immune cells at sites of inflammation. F1000Research. 5, 175 (2016).

[36] López-Lázaro, M. The migration ability of stem cells can explain the existence of cancer of unknown primary site. Rethinking metastasis. Oncoscience. 2, 467-75(2015)

[37] Einstein, A., Podolsky, B. and Rosen, N. Can Quantum-Mechanical Description of Physical Reality Be Considered Complete? Physical Review. 47, 777-780 (1935).

[38] Shu, D. G. et al. Head and backbone of the Early Cambrian vertebrate Haikouichthys. Nature 421, 526-529(2003)

[39] Barrangou, R., Marraffini, L.A. CRISPR-Cas systems: Prokaryotes upgrade to adaptive immunity. Molecular Cell. 54 (2), 234-44(2014).

[40] Piovesan, A., Caracausi, M., Ricci, M., Strippoli, P., Vitale, L., Pelleri, M. C. Identification of minimal eukaryotic introns through GeneBase, a user-friendly tool for parsing the NCBI Gene databank. DNA Research. 22 (6), 495-503(2015).

[41] Turanov, A.A. et al. Genetic code supports targeted insertion of two amino acids by one codon. Science. 323, 259-61(2009).

[42] Vigneron, N. et al. An Antigenic Peptide Produced by Peptide Splicing in the Proteasome. Science. 304, 587-590(2004).

[43] Yamazaki, K. et al. Control of mating preferences in mice by genes in the major histocompatibility complex. Journal of Experimental Medicine. 144, 1324-1335(1976).

[44] Skaletsky, H. et al. The male-specific region of the human $\mathrm{Y}$ chromosome is a mosaic of discrete sequence classes. Nature 423, 825-837(2003)

[45] Sakharkar, M.K., Chow, V.T.,Kangueane, P. Distributions of exons and introns in the human genome. In Silico Biol., 4(4), 387-93 (2004).

[46] Francis, W.R., Wörheide, G. Similar ratios of introns to intergenic sequence across animal genomes. Genome Biol. Evol. 9(6),1582-1598(2017).

[47] Black, D. L. Mechanisms of alternative pre-messenger RNA splicing. Annual Review of Biochemistry. 72 (1), 291-336(2003).

[48] Pan, Q., Shai, O., Lee, L.J., Frey, B.J., Blencowe, B.J. Deep surveying of alternative splicing complexity in the human transcriptome by high-throughput sequencing. Nature Genetics. 40, 1413-1415 (2008).

[49] Schmucker, D. et al. Drosophila Dscam is an axon guidance receptor exhibiting extraordinary molecular diversity. Cell 101, $671-684(2000)$
[50] Sibley, C. R. et al. Recursive splicing in long vertebrate genes. Nature 521 (7552), 371-375(2015)

[51] Gazzoli, I. et al. Non-sequential and multi-step splicing of the dystrophin transcript. RNA Biology, 13, 290-305(2016).

[52] Rodríguez-Trelles, F., Tarrío, R., Ayala, F.J. Origins and evolution of spliceosomal introns. Annu. Rev. Genet. 40, 47-76(2006).

[53] Mourier, T., Jeffares, D.C. Eukaryotic intron loss. Science 300,1393-1393(2003)

[54] Jeffares, D.C., Penkett, C.J., Bähler, J. Rapidly regulated genes are intron poor. Trends in Genetics. 24, 375-8(2008)

[55] Castillo-Davis, C.I., Mekhedov, S.L., Hartl, D.L., Koonin, E.V., Kondrashov, F.A. Selection for short introns in highly expressed genes. Nature Genetics. 31, 415-8 (2002).

[56] Hanada, K., Yang, J. C. Novel biochemistry: Post-translational protein splicing and other lessons from the school of antigen processing. Journal of Molecular Medicine. 83, 420-8(2005).

[57] $\mathrm{Xu}, \mathrm{J}$. et al. Conformational complexity and dynamics in a muscarinic receptor revealed by NMR spectroscopy. Mol Cell. Pii,S1097-2765(2019).

[58] Bürglin, T.R., Affolter, M. Homeodomain proteins: an update Chromosoma. 125 , 497-521 (2016).

[59] Schofield, P.N. Patterns, puzzles and paradigms - The riddle of the homeobox. Trends Neurosci. 10, 3-6 (1987).

[60] de Rosa et al. Hox genes in brachiopods and priapulids and protostome evolution. Nature 399, 772-776(1999).

[61] Ryan, J.F. et al. Pre-Bilaterian Origins of the Hox Cluster and the Hox Code: Evidence from the Sea Anemone, Nematostella vectensis. PLoS ONE 2(1), e153 (2007)

[62] Wickstead, B., Gull, K. The evolution of the cytoskeleton. The Journa of Cell Biology. 194, 513 - 25 (2011).

[63] Fletcher, D.A., Mullins, R.D. Cell mechanics and the cytoskeleton. Nature 463,485-92(2010).

[64] Ferguson-Smith, A.C. Genomic imprinting: the emergence of an epigenetic paradigm. Nature Reviews Genetics. 12 , 565-75 (2011)

[65] Smith, D.J. Human sperm accumulation near surfaces: a simulation study. Journal of Fluid Mechanics. 621, 295(2009)

[66] Baines, H.L., Turnbull, D.M., and Greaves, L.C. Human stem cell aging: do mitochondrial DNA mutations have a causal role? Aging Cell 13,201-205 (2014).

[67] Yonutas, H.M., Pandya, J.D., and Sullivan, P.G. Changes in mitochondrial bioenergetics in the brain versus spinal cord become more apparent with age. J Bioenerg Biomembr. 47, 149-154 (2015).

[68] Murata, T., Ohtsuka, C., Terayama, Y. Increased mitochondria oxidative damage in patients with sporadic amyotrophic lateral sclerosis. Journal of the Neurological Sciences 267, 66-69 (2008).

[69] Peterson, C.M., Johannsen, D.L., and Ravussin, E. Skeletal Muscle Mitochondria and Aging: A Review. Journal of Aging Research. 2012 ID 194821, 1-20 (2012)

[70] Panov, A., Kubalik, N., Zinchenko, N., Hemendinger, R., Dikalov, S. Bonkovsky, H.L. Respiration and ROS production in brain and spinal cord mitochondria of transgenic rats with mutant G93a $\mathrm{Cu} / \mathrm{Zn}$-superoxide dismutase gene. Neurobiology of Disease 44, 53-62 (2011)

[71] Jourdain, A., Martinou, J. Mitochondrial outer-membrane permeabilization and remodelling in apoptosis. The International Journal of Biochemistry \& Cell Biology, 41, 1884-1889 (2009).

[72] Mils, V. et al. Mitochondrial Reshaping Accompanies Neura Differentiation in the Developing Spinal Cord. PLOS ONE 10(5): 0128130

[73] Regenold, W.T., Phatak, P., Makley, M.J. R.D., Kling, M.A. Cerebrospinal fluid evidence of increased extra-mitochondrial glucose metabolism implicates mitochondrial dysfunction in multiple sclerosis disease progression. Journal of the Neurological Sciences $\mathbf{2 7 5}$, 106-112 (2008)

[74] Albanese, M. et al. Cerebrospinal fluid lactate is associated with multiple sclerosis disease progression. Journal of Neuroinflammation 13, 1-7 (2016)

[75] Berger, E. Mitochondrial function controls intestinal epithelia stemness and proliferation. Nature Communications 7, 13171(2016).

[76] Fernández-Moreno, M. et al. mtDNA haplogroup J Modulates telomere length and Nitric Oxide production. BMC Musculoskeletal Disorders, 12, 283(2011).

[77] Dzeja, P.P., Bortolon, R., Perez-Terzic, C., Holmuhamedov, E.L., and Terzic, A. Energetic communication between mitochondria and nucleus directed by catalyzed phosphotransfer. PNAS $\mathbf{9 9}$ 10156-10161 (2002).

\section{Volume 8 Issue 11, November 2019}

www.ijsr.net 


\section{International Journal of Science and Research (IJSR) \\ ISSN: 2319-7064}

ResearchGate Impact Factor (2018): 0.28 | SJIF (2018): 7.426

[78] Picarda, M., and McEwen, B.S., Mitochondria impact brain function and cognition. PNAS 111, 7-8 (2014).

[79] Anderson, S. et al. Sequence and organization of the human mitochondrial genome. Nature 290, 457-65(1981).

[80] Johnston, I. G., Williams, B. P. Evolutionary Inference across Eukaryotes Identifies Specific Pressures Favoring Mitochondrial Gene Retention. Cell Systems. 2, 101-11(2016).

[81] Kondo, R., Matsuura, E.T., Chigusa, S.I. Further observation of paternal transmission of Drosophila mitochondrial DNA by PCR selective amplification method. Genetical Research. 59, 81-4(1992).

[82] Meusel, M. S., Moritz, R. F. A. Transfer of paternal mitochondrial DNA during fertilization of honeybee (Apis mellifera L.) eggs. Current Genetics. 24 (6), 539-43(1993).

[83] Fontaine, K.M., Cooley, J. R., Simon, C. Evidence for Paternal Leakage in Hybrid Periodical Cicadas (Hemiptera: Magicicada spp.). PLOS ONE. 2 (9): e892(2007).

[84] Zhao, X., Li, N., Guo, W. et al. Further evidence for paternal inheritance of mitochondrial DNA in the sheep (Ovis aries). Heredity. 93 (4), 399-403(2004).

[85] Steinborn, R. et al. Composition of parental mitochondrial DNA in cloned bovine embryos. FEBS Letters. 426 (3), 352-6(1998).

[86] Schwartz, M., Vissing, J. Paternal Inheritance of Mitochondrial DNA. New England Journal of Medicine. 347 (8), 576-80(2002).

[87] St John, J., Sakkas, D., Dimitriadi, K. et al. Failure of elimination of paternal mitochondrial DNA in abnormal embryos. Lancet $\mathbf{3 5 5}, 200$ (2000).

[88] Houshmand, M., Holme, E., Hanson, C., Wennerholm, U.B., Hamberger, L. Is paternal mitochondrial DNA transferred to the offspring following intracytoplasmic sperm injection? J Assist Reprod Genet 14,223-7(1997).

[89] Felgenhauer, K. Protein size and CSF composition. Klin. Wochenschr. 52, 1158-64 (1974)

[90] Merril, C.R., Goldman, D., Sedman, S.A., Ebert, M.H. Ultrasensitive stain for proteins in polyacrylamide gels shows regional variation in cerebrospinal fluid proteins. Science. 211, 1437-8 (1981).

[91] Saunders, N.R., Habgood, M.D., Dziegielewska, K.M. Barrier mechanisms in the brain, I. Adult brain. Clin. Exp. Pharmacol. Physiol. 26 (1), 11-9(1999).

[92] Chazal, J. Anatomy and physiology of cerebrospinal fluid. European Annals of Otorhinolaryngology, Head and Neck Diseases. 128, 309-316(2011).

[93] Klarica, M., Orešković, D. A new look at cerebrospinal fluid movement. Fluids Barriers CNS. 11, 16(2014).

[94] Silver,D. et al. Mastering the game of Go with deep neural networks and tree search. Nature 529, 484-489(2016).

[95] Larina, I.M. et al. Protein expression changes caused by spaceflight as measured for 18 Russian cosmonauts. Sci Rep. 7, 8142(2017).

[96] Wickramasinghe, C. Bacterial morphologies supporting cometary panspermia: a reappraisal. International Journal of Astrobiology 10 (1), 25-30(2011).

[97] Joyce, G.F. The antiquity of RNA-based evolution. Nature 418 (6894), 214-221(2002).

[98] Wächtershäuser, G. Life as We Don't Know It. Science 289 (5483), 1307-1308 (2000).

[99] Wilde, S.A., Valley, J.W., Peck, W.H. and Graham, C.M. Evidence from detrital zircons for the existence of continental crust and oceans on the Earth 4.4 billion years ago. Nature 409, 175-178(2001).

[100] Walden, P. Ueber die gegenseitige Umwandlung optischer Antipoden. Berichte der deutschen chemischen Gesellschaft. 29, 133-138(1896). 